University of Louisville

ThinkIR: The University of Louisville's Institutional Repository

$12-2007$

\title{
Molecular interactions between the Wilms' tumor transcription factor and the aryl hydrocarbon receptor during nephrogenesis and its impact on fetal programming of renal disease.
}

Adrian Nanez

University of Louisville

Follow this and additional works at: https://ir.library.louisville.edu/etd

\section{Recommended Citation}

Nanez, Adrian, "Molecular interactions between the Wilms' tumor transcription factor and the aryl hydrocarbon receptor during nephrogenesis and its impact on fetal programming of renal disease." (2007). Electronic Theses and Dissertations. Paper 1039.

https://doi.org/10.18297/etd/1039

This Doctoral Dissertation is brought to you for free and open access by ThinkIR: The University of Louisville's Institutional Repository. It has been accepted for inclusion in Electronic Theses and Dissertations by an authorized administrator of ThinkIR: The University of Louisville's Institutional Repository. This title appears here courtesy of the author, who has retained all other copyrights. For more information, please contact thinkir@louisville.edu. 


\title{
IMOLECULAR INTERACTIONS BETWEEN THE WILMS' TUMOR TRANSCRIPTION FACTOR AND THE ARYL HYDROCARBON RECEPTOR DURING NEPHROGENESIS AND ITS IMPACT ON FETAL PROGRAMMING OF RENAL DISEASE
}

\author{
By \\ Adrian Nañez \\ B.S., University of Texas, 2002
}

A Dissertation Submitted to the Faculty of the Graduate School of the University of Louisville In Partial Fulfillment of the Requirements For the Degree of

Doctor of Philosophy

Department of Biochemistry and Molecular Biology

University of Louisville, Louisville, KY

December 2007 
MOLECULAR INTERACTIONS BETWEEN THE WILMS' TUMOR

TRANSCRIPTION FACTOR AND THE ARYL HYDROCARBON RECEPTOR

DURING NEPHROGENESIS AND ITS IMPACT ON FETAL PROGRAMMING

OF RENAL DISEASE

By

Adrian Nañez

B.S., University of Texas, 2002

A Dissertation Approved on

November $16^{\text {th }}, 2007$

By the Following Dissertation Committee

Kenneth S. Ramos, Ph.D., Dissertation Director

Thomas A. Geoghegan, Ph.D.

Russell A. Prough, Ph.D.

Carolyn A. Klinge, Ph.D.

Lawrence A. Shoemaker, M.D. 
Copyright 2007 by Adrian Nañez

All rights reserved 


\section{DEDICATION}

This dissertation is dedicated to my parents Mr. and Mrs. Jesus Sergio Nañez; my brothers Sergio, Rolando, Marcial, Daniel; my loving family; and my loyal friends Raymond, Katie, Dave, Rachel, Ryan, Marlene, Jeannie, Vilius, Ivo, John, Marvin, Kim, Monica, John Paul, Beau, Brian, Olivia, Clay, Marge, Ashley, Krista, Dan, Matt, Glenn, Greg, Megan, and Susan. 


\section{ACKNOWLEDGEMENTS}

I would like to thank Mr. Benito Garcia and Eduardo Villa for providing me with my first exposure to scientific method. I would like to thank Dr. John Richburg for providing my initial scientific mentoring and for his continued friendship. My thanks go out to Dr. Kenneth $\mathrm{S}$. Ramos for training me in scientific rigor and constantly pushing my limits in all facets of research training Science is a team effort and without my coworkers Marlene, Jeannie, Vilius, Ivo, Diego, and Hadi my tenure would not have been as enjoyable. I would like to thank my friends and my family for their steadfast loyalty and unwavering kindness. Lastly, I would like to thank my parents for instilling in me a hard work ethic and a strong sense of duty. 


\title{
ABSTRACT
}

\author{
MOLECULAR INTERACTIONS BETWEEN THE WILMS' TUMOR \\ TRANSCRIPTION FACTOR AND THE ARYL HYDROCARBON RECEPTOR \\ DURING NEPHROGENESIS AND ITS IMPACT ON FETAL PROGRAMMING \\ OF RENAL DISEASE
}

Adrian Nañez

December 3, 2007

Embryonic development requires the orchestration of temporally precise genetic events that culminate in the formation of a complete organism. The molecular mechanisms responsible for ontogenesis are regulated by environmental and somatic factors in utero that activate or repress the expression of numerous genetic elements resulting in fetal programming of adult diseases. The aryl hydrocarbon receptor (AHR) is an important nuclear transcription factor both during embryogenesis and throughout maturity in multiple organisms. Building upon interesting studies establishing a direct, novel link between AHR and post-transcriptional regulation of the Wilms' tumor suppressor (WT1) gene, the overall goal of the project was to elucidate the role of AHR in the regulation of WT1 during nephrogenesis. The evidence shows that 
in utero exposure to environmentally relevant exposures of benzo(a)pyrene (BaP), a polycyclic aromatic hydrocarbon (PAH) and AHR ligand, results in AHR allele-specific deficits in renal development manifested as decreased numbers of glomeruli, induction in urinary albumin, and podocytopenia. These findings were consistent with metanephric and organ culture data where PAHs caused AHR allele-specific reduction in renal cell differentiation markers, dysregulation of WT1 mRNA splice variants, and decreases in direct WT1 transcriptional targets.

Observed deficits were linked to disruption of constitutive AHR signaling, as siRNA-mediated AHR degradation reproduced similar effects. Collectively, this evidence defined a novel role for the AHR in renal development and in fetal programming of $\mathrm{PAH}$-induced environmental disease. 
TABLE OF CONTENTS

PAGE

ACKNOWLEDGEMENTS

iv

ABSTRACT

V-vi

CHAPTER 1

INTRODUCTION

1

CHAPTER 2

Summary

Introduction

Materials and Methods

Results

52

Discussion

CHAPTER 3

Summary

71

$\begin{array}{ll}\text { Introduction } & 72\end{array}$

Materials and Methods

Results

Discussion 


\section{CHAPTER 4}

Summary

Introduction

Materials and Methods

Results

Discussion

CHAPTER 5

DISCUSSION

REFERENCES

183

CURRICULUM VITAE

203 


\section{CHAPTER 1 \\ INTRODUCTION}

Embryonic development requires the orchestration of temporally precise genetic and genetic events that culminate in the formation of a complete organism. The molecular mechanisms responsible for ontogenesis are regulated by environmental and somatic factors in utero that activate or repress the expression of numerous genetic elements. The aryl hydrocarbon receptor (AHR) is an important nuclear transcription factor both during embryogenesis and throughout maturity in multiple organisms. The discovery of potent, environmental chemicals that act as AHR ligands has revealed that dysregulation of AHR function during embryonic development can have deleterious effects. Of relevance to this project, from a medical perspective, are epidemiological reports that maternal exposure to environmental tobacco smoke, a complex mixture of chemicals containing high concentrations of ligands of the AHR such as polycyclic aromatic hydrocarbons (PAH), mediates developmental abnormalities (Sasaki et al., 2006). Also of note is that children of parents who work in occupations associated with increased exposure to AHR ligands have a higher incidence of Wilms' tumors (Olshan et al., 1990). The discovery of novel functions of AHR during development, coupled to molecular interactions of AHR 
with developmental transcription factors highlight a potential mechanism for compromised development following disruption of AHR signaling. This dissertation focuses on the interactions between AHR and WT1 in the regulation of nephrogenesis.

Renal development proceeds in three morphologically distinguishable stages: the pronephros, mesonephros, and metanephros. During embryogenesis, the kidney derives from the nephric ridge in the intermediate mesoderm. At gestational day 8 in mice and day 22 in humans, the pronephric duct gives rise to the pronephros which elongates to form the Wolffian duct. A morphologically distinct mesonephros appears in mice at gestational day 9.5 and day 24 in humans. During mesonephric development, glomeruli are linked to the Wolffian duct forming immature nephrons. Shortly thereafter, the mesonephric ducts and remnants of the tubular structure are incorporated into development of the male genital system and disappear altogether in females (Sakurai and Nigam, 2000).

The metanephros (also referred to as the permanent kidney) develops at gestational day 11 in mice and day 28 in humans. During metanephric development, the Wolffian duct develops into the ureteric bud which then enters the nephrogenic mesenchyme and stimulates condensation of mesenchymal cells around the invading ureteric bud. The reciprocal interactions between the invading ureteric bud and the metanephric mesenchyme initiate mesenchymal- 
epithelial cell transformation (MET) wherein epithelial cells are stimulated to differentiate into the precluding structures for glomerular development (Kuure et al., 2000). Both proximal and distal tubules are derived from mesenchymal cells receiving differentiation signals from the invading ureteric bud. Reciprocal signals stemming from the induced mesenchyme stimulate branching tubulogenesis of the ureteric bud culminating in development of the collecting duct system.

Of importance to our work are the mechanisms that govern the mesenchymal-to-epithelial transformation and the resulting influence on metanephric development and renal function. Growth of the ureteric bud from the Wolffian duct is regulated by the Wilms' tumor (WT1), Pax2, Lim1, Sall1, and Eya1 transcription factors in cooperation with the ancillary proteins formin, Gdnf, Ret, and Gfra1 (for review see (Kuure et al., 2000). Homozygous deletion of only one regulator is sufficient to inhibit ureteric bud outgrowth causing bilateral renal agenesis or severe forms of renal dysgenesis (Kreidberg et al., 1993a; Maas et al., 1994; Pichel et al., 1996; Sanchez et al., 1996; Schuchardt et al., 1994; Shawlot and Behringer, 1995a; Torres et al., 1995; Treanor et al., 1996; Xu et al., 1999). Pax2-null mice do not develop the caudal portion of the Wolffian duct and do not develop the ureteric bud (Torres et al., 1995). In WT1-null mice, ureteric bud growth is stunted resulting in renal aplasia (Kreidberg et al., 1993a). Sall1 deficiency does not alter ureteric bud growth, but inhibits invasion of the metanephric mesenchyme resulting in bilateral renal aplasia (Nishinakamura et 
al., 2001). Gdnf produced in the metanephric mesenchyme functions with Ret to promote ureteric bud outgrowth. Disruption of either Gdnf or Ret alters renal branching morphogenesis due to improper ureteric bud outgrowth (Cacalano et al., 1998; Schuchardt et al., 1994).

A cascade of genetic events initiated by transcriptional control from master switches including WT1, Lim1, and Pax2 transcription factors regulate MET (Figure 1) (Kuure et al., 2000). WT1 is a known regulator of Pax2 which in turn binds to the 5' promoter of Gdnf to induce transcription (Brophy et al., 2001). Lim1-null mice develop a mesonephros, but are devoid of adult kidneys due to deficits in Pax2 transcriptional control (Tsang et al., 2000). While the importance of master switch transcriptional regulators is well-established, the mechanisms controlling their function and the downstream consequences are poorly understood.

Other influences in ureteric bud morphogenesis are cellular interactions involving extracellular matrices (ECM) from both the ureteric bud and the metanephric mesenchyme. ECM protein expression is tightly regulated via growth factor signaling pathways which, in turn, are regulated by master switch transcription factors. Laminins compose the majority or the mature basement membrane (Engel, 1993), and have been shown to participate in branching morphogenesis and tubulogenesis. Exposure to anti-laminin5 blocking antibody inhibits renal development of cultured metanephroi by preventing association 
Figure 1. Representation of ureteric bud and metanephric mesenchyme interactions in nephron development. Renal cell differentiation requires the sequential activation of gene expression from several key regulators and downstream effectors. These include secreted factors (Wnt, FGF, BMP, and GDNF), receptors (c-ret), oncogenes, transcription factors (WT1, Pax2, Lim1), enzymes (HS2-sulfotransferase), signal transducers (RARa), proteolytic enzymes, extracellular matrix (integrin $\alpha 8$ ), endothelium attractant (VEGF), and glomerular regulators (PDGF, and nephrin) (Kuure et al., 2000). 
Figure 1

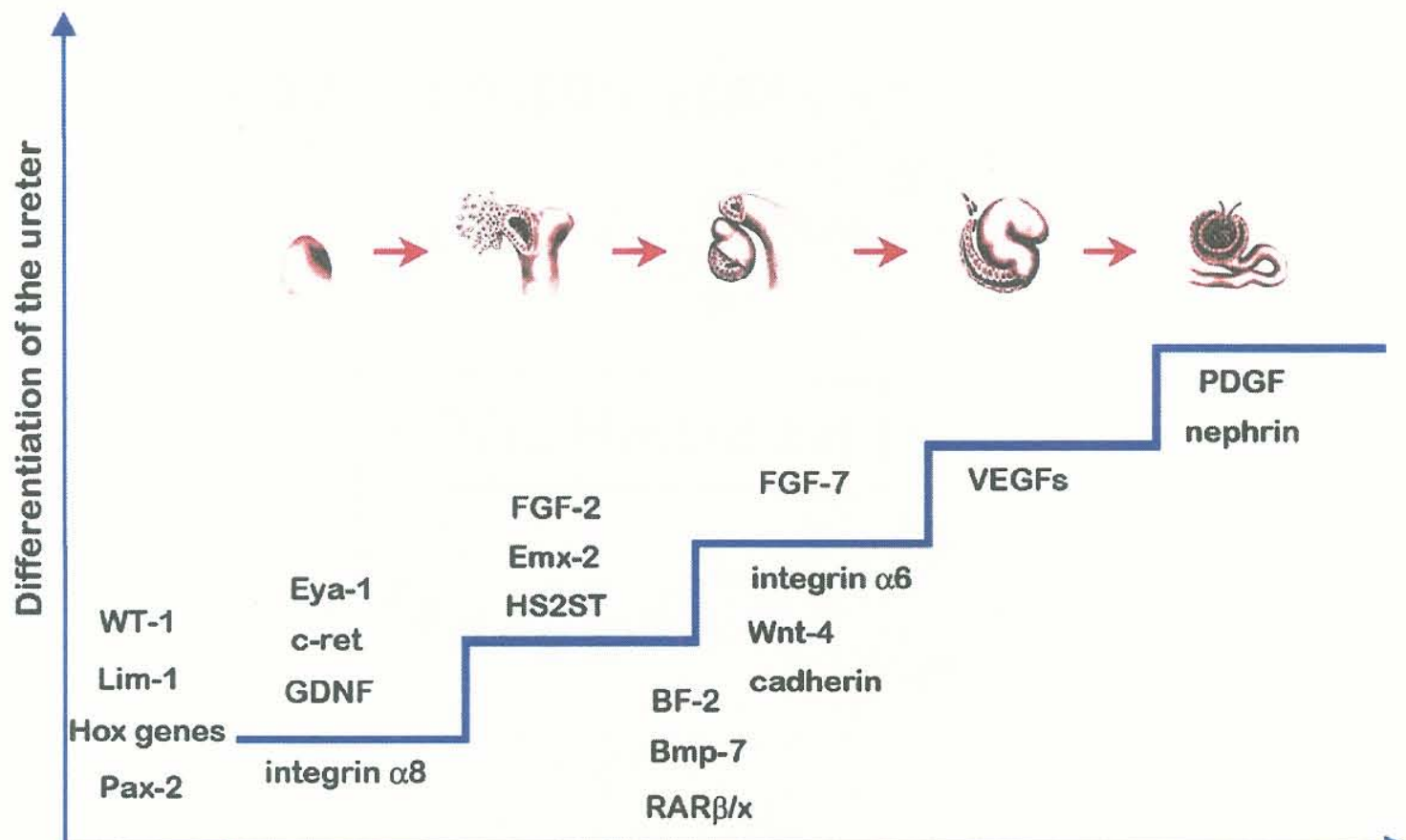

Differentiation of the mesenchyme 
with $\alpha_{3} \beta_{1}$ and $\alpha_{6} \beta_{4}$-integrin receptors (Zent et al., 2001). Laminin 1, consisting of $\alpha-, \beta-$, and $y$-subunits, is a critical component of epithelial cell polarization chiefly expressed in comma and S-shaped bodies (Durbeej et al., 1996; Ekblom, 1993). In cultured metanephroi, mesenchymal cell excreted nidogen interacts with the laminin $1 \gamma_{1}$-subunit to facilitate epithelial cell polarization through a mechanism dependent upon dystoglycan and $\alpha_{6} \beta_{1}$-integrin (Ekblom et al., 1994). Exposure to antibodies generated against $\alpha_{1}$, or $\alpha_{1} \beta_{1} \gamma_{1}$-subunits inhibits development of cultured metanephroi either through binding of the dystroglycan membrane complex or the $\alpha_{6} \beta_{1}$-integrin receptor (Sonnenberg et al., 1990).

Cells stemming from the ureteric bud must digest ECM proteins to facilitate invasion. Both matrix metalloproteases (MMPs) and serine proteases are known to facilitate renal development. Membrane-type matrix 1 (MT-1-MMP) and MMP-2 oligonucleotide antisense inhibits metanephric development dependent on the TIMP-2 inhibitor activity (Kanwar et al., 1999; Ota et al., 1998) While t-PA and u-PA serine proteases are expressed in developing metanephroi, and have been shown to participate in tubulogenesis in organ culture, mouse models deficient in expression of either protein have a normal renal phenotype (Carmeliet et al., 1994). Thus, renal morphogenesis requires the function of master switch transcription factors which directly or indirectly regulate intra/extracellular signaling that facilitate renal cell differentiation, branching morphogenesis, and ultimately nephrogenesis. 
The Wilms' tumor suppressor transcription factor (WT1), in concert with a variety of transcriptional co-regulators, guides renal development through both direct and indirect modulation of target genes necessary for renal cell differentiation including syndecan1(Synd1), Pax2, E-cadherin (E-cad), insulin-like growth factor II (Igf2r), epidermal growth factor receptor (Egfr) and retinoic acid receptor- $\alpha$ (Rara) (Hosono et al., 2000; Hosono et al., 1999; Ryan et al., 1995). The WT1 gene encodes a $\mathrm{Cis}_{2}-\mathrm{His} 2$ zinc fingered protein that can regulate biological events via transcriptional activation of target genes or by association with factors participating in RNA processing. The WT1 gene was first characterized as one of the genetic causes of the pediatric nephroblastoma Wilms' tumor, which is histologically characterized by cellular populations of epithelial, stromal, and undifferentiated phenotype (Bennington and Beckwith, 1975). While WT1 is only inactivated in 15\% of Wilms' tumors, dysregulation of WT1 function results in clinical manifestation of developmental deficiencies affecting genitourinary development such as WAGR (Wilms' tumor, aniridia, genitourinary defects and mental retardation), Beckwith-Wiedemann, DenysDrash, and Frasier syndrome (for review see(Lee and Haber, 2001)).

Of importance to our work is the ability of WT1 to function as a "master switch" regulating mesenchymal-epithelial differentiation in renal development (Avner, 1993). In both humans and mice, WT1 protein is expressed in the condensing mesenchyme, renal vesicle, and glomerular epithelium of developing kidney (Buckler et al., 1991; Pritchard-Jones et al., 1990). In adulthood, visceral 
epithelial cells (podocytes) of the kidney and Sertoli cells of the seminiferous tubules in the testis are the only populations that express WT1. Homozygous WT1-/- mice do not develop normally, die in utero, and do not differentiate into metanephric kidney from the existing pronephros (Kreidberg et al., 1993b).

WT1 activity is regulated in cis by different ratios of its own splice variants or in trans by proteins such as bone marrow zinc finger 2 (BMZF2), Pax2, and Par4 (Hohenstein and Hastie, 2006; Lee et al., 2002). WT11 protein expression varies from 36 possible isoforms resulting from alternative splicing, start codons, and RNA editing (Figure 2) (Hohenstein and Hastie, 2006). Perhaps the most studied regulatory mechanism involves the formation of WT1 1 KTS splice variants. KTS splice variants are formed at a site in exon 9 via the insertion of a lysine-threonine-serine (KTS) between the third and fourth zinc finger. The resulting change targets - KTS WT1 protein expression for the nucleus and modifies DNA binding specificity leading to activation and/or repression of different genetic elements (Menke et al., 1998b; Niksic et al., 2004). WT1 protein from the +KTS mRNA transcript localizes to the nucleus and is thought to participate in RNA processing via interactions with known splicing factors such as U2AF65 (Davies et al., 1998; Ladomery et al., 2003; Larsson et al., 1995). In mouse models, deletion of either the -KTS or the +KTS is lethal soon after birth due to distinct renal deficits (Hammes et al., 2001). In humans, reduced WT1+KTS mRNA isoform results in severe kidney and gonad developmental deficits, collectively known as Frasier syndrome (Barbaux et al., 1997). Similarly, 
Figure 2. Schematic representation of the WT1 gene. Accounting for alternative transcriptional start sites and mRNA splicing, the WT1 locus encodes for at least a theoretical maximum of 36 isoforms. Alternative start codons, exons, splice sites, and RNA editing are depicted in red. Functional domains of known biological importance are depicted in green (Hohenstein and Hastie, 2006). 
Figure 2

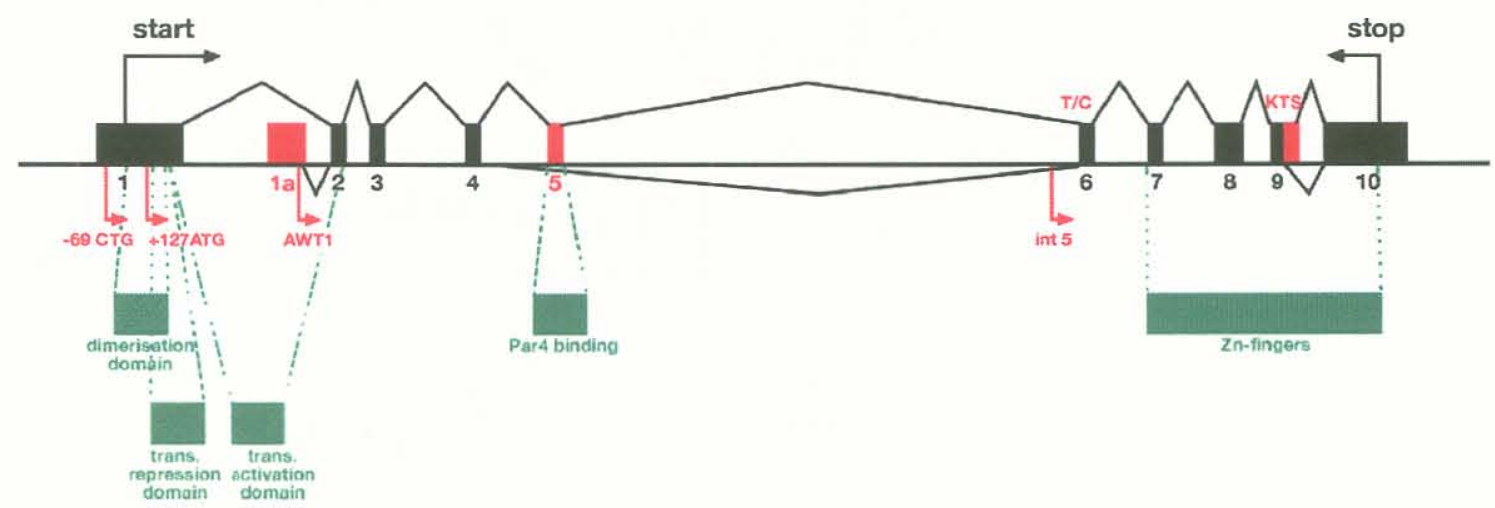


mouse models with homozygous deletion of the -KTS WT1 variant results in small kidneys, reduced glomeruli number, a decrease in nephrogenic area, as well as streak gonads (Hammes et al., 2001). +KTS-null mice achieve a higher degree of renal maturation, compared to -KTS $-/$ - mice, characterized by immature glomeruli and undifferentiated podocyte cell populations resulting in renal nephropathy evidenced by hematuria (Hammes et al., 2001). This demonstrates that the integrity of WT1 function as regulated by mRNA splice variant ratio is critical to renal development.

Addition of 17 amino acids comprising the entirety of exon 5 ( $\pm 17 \mathrm{aa})$ occurs in a region chiefly thought to regulate transactivation with regulatory partners and nuclear translocation (Roberts, 2005; Wang et al., 1995). Insertion or deletion of 17 aa does not alter renal development morphologically, and to date the impact of these isoforms on WT1 function remains uncertain. Overexpression of +17 aa has been reported in human Wilms' tumor (Simms et al., 1995). In cell culture models, +17 aa overexpression alters cellular morphology and induces proliferation (Hewitt and Saunders, 1996). In lymphoma cell lines +17 aa overexpression inhibits apoptosis by repressing expression the pro-apoptotic protein Bak (Ito et al., 2006). Our work shows that induction of +17aa WT1 protein correlates with loss of renal cell differentiation markers Igf1r, Wnt4 and E-cad (Falahatpisheh and Ramos, 2007). 
Yet another regulatory domain occurs at the $\mathrm{N}$-terminal residues $1-182$ which encode a dimerization region implicated in the regulatory mechanism exerted by dominant negative mutants (Englert et al., 1995; Reddy et al., 1995). To date, the mechanisms regulating WT1 function are poorly understood. The majority of evidence supports the theory that WT1 is regulated by relative abundance of mRNA isoforms resulting in proteins that function to either repress or promote transcription of genetic targets necessary for renal cell differentiation. The relative contribution that +KTS plays in RNA processing has yet to be determined. The molecular mechanisms regulating WT1 transcription, splicing, and overall function remain uncertain. Work in our laboratory suggests that integrity of AHR signaling functions directly and/or indirectly in regulation of WT1 mRNA ratios.

The aryl hydrocarbon receptor was the first characterized member of the basic helix loop helix- Per Arnt Sim (bHLH-PAS) family of transcription factors (Figure 3). The PAS domain is characterized by a region of homology between the founding members Per (the protein product of the Drosophila Period gene) (Reddy et al., 1986), ARNT (the AHR nuclear translocator) (Hoffman et al., 1991), and Sim (the product of the Drosophila-Single minded locus) (Nambu et al., 1991). Eukaryotic PAS domains serve as recognition sites for interactions with other PAS proteins, cellular chaperones, or in the case of the AHR, a regulatory ligand binding site. The bHLH domain also participates in recognition of other bHLH and facilitates recognition of the major groove of target genetic 
Figure 3. Members of the PAS family. Domain structures for the founding members PER, ARNT, and SIM are shown as well as others including AHR, HIF1, TRH, and SIMILAR The basic region is depicted in blue, basic helix-loophelix in grey, PAS domain in red, and transactivation domain in yellow. 
Figure 3

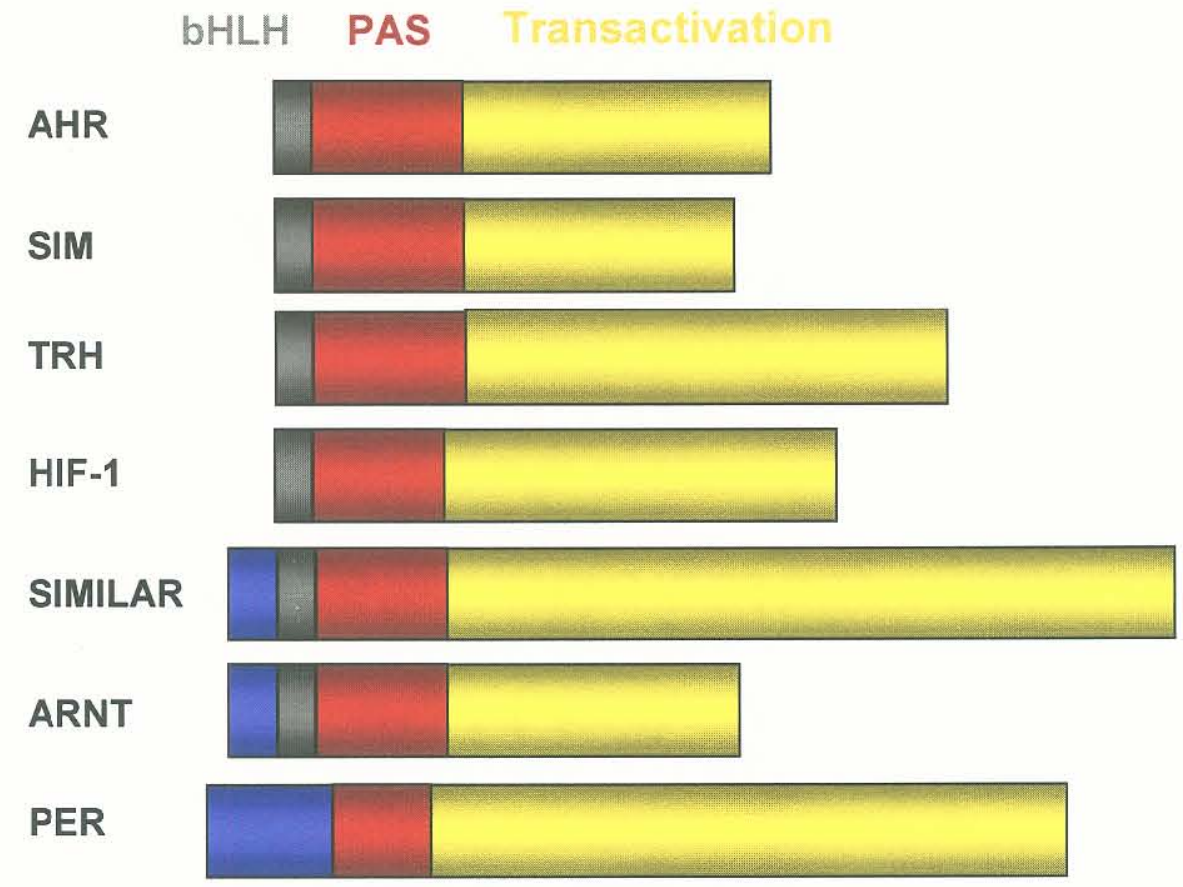


elements (Murre et al., 1994). While bHLH-PAS proteins share similar structure and function, the AHR is the only member known to be regulated by ligand activation (Figure 4).

The AHR was initially characterized due to its participation in the induction of xenobiotic metabolizing enzymes (XMEs) necessary for removal of exogenous compounds. Early work showed that response to xenobiotics such as polycyclic aromatic hydrocarbons (PAHs), known inducers of XMEs, varied between strains of mice that were highly responsive (C57BL/6J) and nonresponsive (DBA/2) with respect to induction of XME machinery. Genetic manipulation creating congenic strains expressing different $A H R$ alleles identified a single autosomal locus coined AH locus (Goujon et al., 1972; Nebert et al., 1972). However, it was not until the development of high-affinity radioligands, AHR-specific antibodies, and purified receptor fractions that AHR-ligand interactions were deduced.

A combination of various techniques has led to our current understanding of AHR biology in which ligand binding results in AHR disassociation from two molecules of the chaperone heat shock protein 90 (HSP90), binds the Xassociated protein 2 (XAP2) and p32 (Figure 5). The resulting complex exposes a nuclear localization sequence that facilitates nuclear translocation. This complex, in turn, binds to the aryl hydrocarbon receptor nuclear translocator (ARNT) resulting in DNA binding to sequences containing 5'-GCGGGGGCG-3' and $(\text { TCC })_{n}$ repeats (Denison and Nagy, 2003). Translocation of the AHR-ligand 
Figure 4. Molecular structure of the AHR. The nuclear localization (NLS), bHLH, PAS, and transactivation (TAD) domains are labeled. Areas characterized for dimerization, ligand binding, and repressor binding are labeled and depicted with lines. Cys 216 has been highlighted as a key residue for DNA binding and Ala 374 for high affinity ligand binding. 
Figure 4

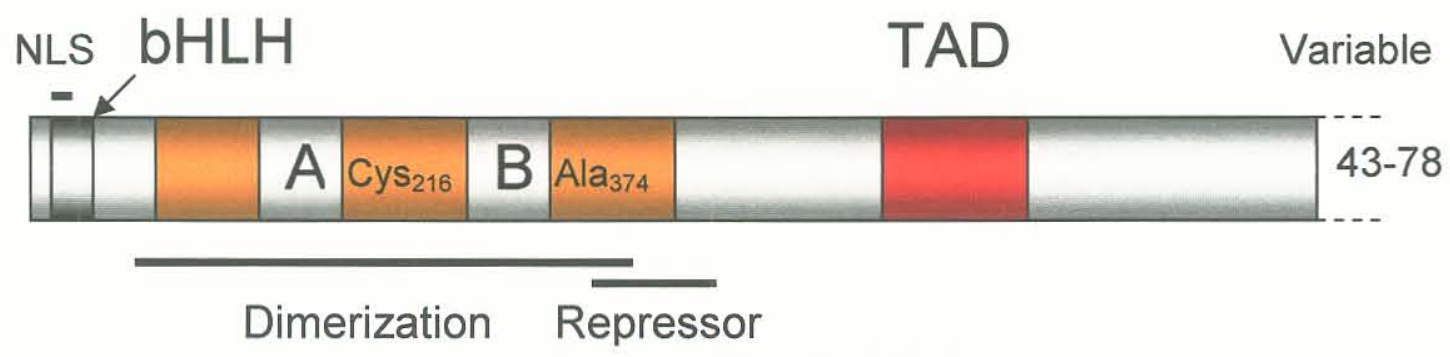


Figure 5. The molecular mechanism of AHR-regulated gene expression as mediated by ligand activation. Ligand binding results in AHR disassociation from two molecules of the chaperone heat shock protein 90 (HSP90), binds the X-associated protein 2 (XAP2) and p32. The resulting complex exposes a nuclear localization sequence that facilitates nuclear translocation. This complex in turn binds to the aryl hydrocarbon receptor nuclear translocator (ARNT) resulting in DNA binding to AHR response element (AHRRE) sequences containing 5'-GCGGGGGCG-3' and (TCC) ${ }_{n}$ repeats. Translocation of the Ahrligand complex triggers proteolytic degradation. 
Figure 5

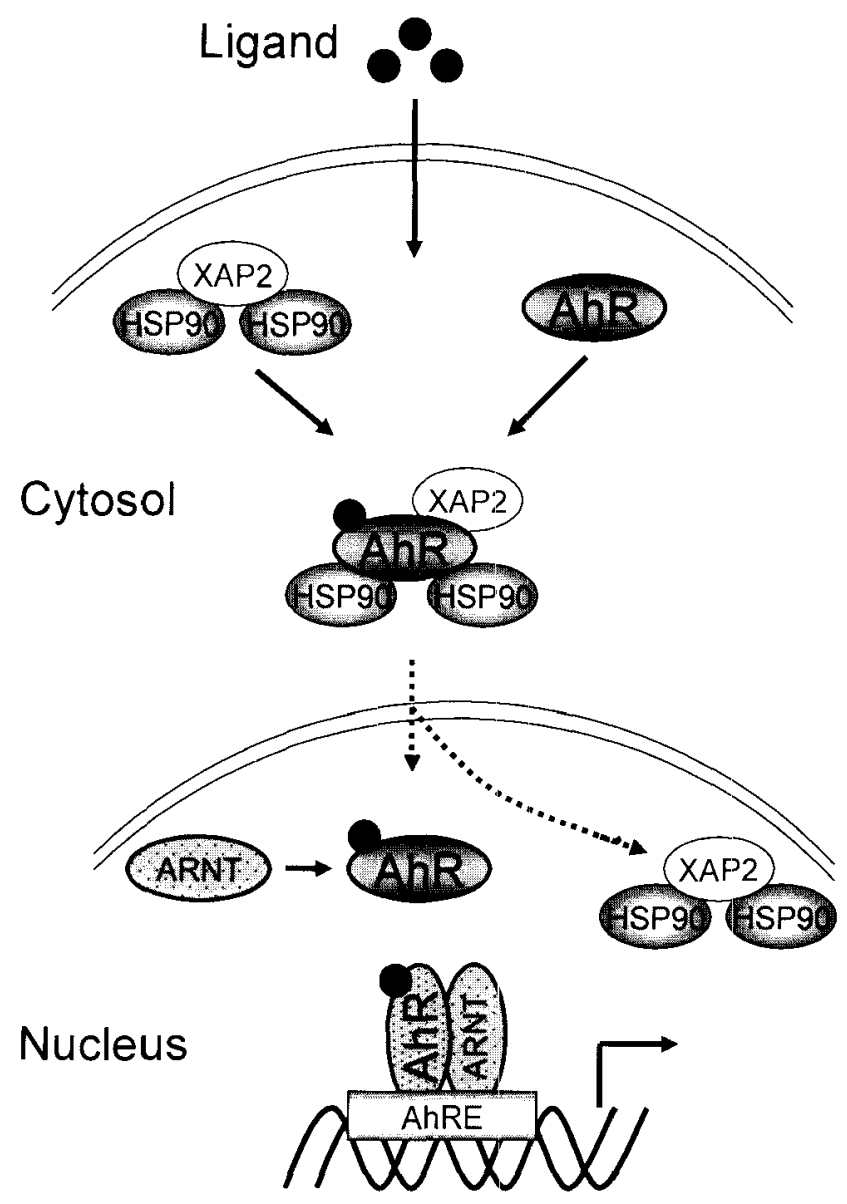


complex to the nucleus can also trigger proteolytic degradation regardless of ARNT binding (Roberts and Whitelaw, 1999). AHR is also regulated by negative feedback inhibition in which the nuclear AHR complex activates transcription of the AHR repressor protein (AHRR) responsible for binding the xenobiotic response element (XRE) (Mimura et al., 1999).

The induction of XMEs resulting from AHR-ligand binding is the most widely studied function of AHR. AHR interacts with xenobiotic response elements (XREs) in the regulatory regions of genes such as cytochrome P450 1A (CYP1A1), CYP1A2, CYP1B1, glutathione-S-transferase Ya (GSTYa) and UGT1A6 NAD(P)H-quinone oxidoreductase which, in turn, function to metabolize PAHs into compounds modified for excretion via glutathione conjugation. An example relevant to our work involves the biotransformation of benzo(a)pyrene (BaP), a common environmental pollutant that among other effects acts as an AHR ligand (Figure 6). Upon exposure to $\mathrm{BaP}, \mathrm{P} 450$ expression is induced to catalyze the epoxidation of the parent hydrocarbon into $\mathrm{BaP} 7,8$ epoxide and then into BaP 7,8 diol-9,10 epoxide (Parkinson, 2001). While these metabolic steps are necessary for clearance of the hydrocarbon, they also activate $\mathrm{BaP}$ into compounds that exert deleterious effects ranging from DNA mutagenesis, oxidative stress, protein modifications, and disruption of molecular signaling pathways (for review see (Miller and Ramos, 2001a). 
Figure 6. Metabolic activation of $\mathrm{BaP} . \mathrm{BaP}$ is metabolized into $\mathrm{BaP} 7,8$ epoxide by cytochrome P450's. Hydrolysis mediated by epoxide hydrolase (EH) creates BaP 7,8 diol which is further metabolized by cytochrome P450's into the BaP 7,8 diol-9,10 epoxide. 
Figure 6<smiles>Cc1ccc2ccc3c4ccccc4cc4ccc1c2c43</smiles>

Benzo(a)pyrene

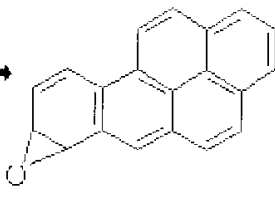

Benzo(a)pyrene 7,8 epoxide

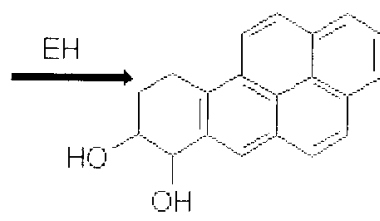

Benzo(a)pyrene 7.8 diol

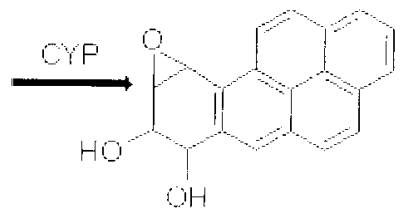

Benzorapylene 7.8 diol-9.10 epoxide 
AHR ligands are categorized as either synthetic in nature (resulting from a nonbiological process such as combustion or de novo synthesis) (Figure 7) or as naturally occurring (synthesized as part of a biological process) (Figure 8).

Further classification into exogenous and or endogenous is difficult due to dietary ligands that are technically exogenous in nature, but that are transformed into ligands by natural biological processes. The high affinity ligands such as $2,3,7,8$ tetrachlorodibenzo-p-dioxin (TCDD), BaP and 3-methylcolanthrene (3MC) are synthetic in nature. Synthetic ligands bind AHR with high affinities in the pM to $\mathrm{nM}$ range whereas naturally occurring ligands such as the tryptophan metabolites bind with lower affinities in the $\mathrm{nM}$ to $\mu \mathrm{M}$ range.

Historically, studies have focused on the ubiquitous high affinity ligands and the resulting regulation of AHR biology, however evolutionary comparison has shown that the AHR gene is conserved across both vertebrate and invertebrate species of which many are not exposed to classical aromatic ligands (Hahn, 2002; Hahn et al., 2006). This poses the question: Are there true endogenous ligands that regulate AHR activity?

The first report of a candidate endogenous AHR ligand came from studies in which Hepa1c1c7 cells were exposed to hemin, bilirubin and biliverdin Bilirubin induced CYP1A1 mRNA and 7-ethoxyresorufin-O-deethylase (EROD) activity in a dose-dependent manner (Sinal and Bend, 1997). In agreement with 
Figure 7. Structures of selected classical AHR ligands that are considered synthetic in nature. All are known inducers of AHR-dependent gene expression (Denison and Nagy, 2003). 
Figure 7

\section{"Classical"AhR Ligands and CVPlAl Inducers}<smiles>Clc1cc2c(cc1Cl)Oc1cc(Cl)c(Cl)cc1O2</smiles><smiles>Clc1ccc(-c2cc(Cl)c(Cl)c(Cl)c2)cc1Cl</smiles><smiles>Clc1cc2oc3cc(Cl)c(Cl)cc3c2cc1Cl</smiles>

2.3.7.8-Tetrachlorodibenzo-p-dioxin<smiles>Cc1ccc2cc3c4c(cc5ccccc53)CCc4c2c1</smiles>

3-Methylcholanthrene

3,4,3',4,5-Pentaclilorobiphenyl<smiles>c1ccc2c(c1)cc1ccc3cccc4ccc2c1c34</smiles>

Benzolaipvrene
23,7,8-Tetrachlorodibencoluran<smiles>O=c1cc(-c2ccccc2)oc2ccc3ccccc3c12</smiles>

B-Vardithoflavone 
Figure 8. Structures of AHR ligands categorized as naturally occurring. All are derivatives arising from metabolism of tryptophan by mammalian enzymes (Denison and Nagy, 2003). 
Figure 8<smiles>CCCc1c[nH]c2ccccc12</smiles>

Tryptamine<smiles>O=C(O)Cc1c[nH]c2ccccc12</smiles>

Indoleacetic Acid<smiles>Nc1ccccc1C(=O)CC(N)C(=O)O</smiles>

L-Kynurenine<smiles>C=C(O)C(=O)CC1CNc2ccccc21</smiles>

Indole Pyruvic Acid 
studies identifying tryptophan metabolites as AHR ligand, aspartate aminotransferase expressed in mouse heart tissue activated AHR signaling by converting L-tryptophan into indole-3-pyruvate (Bittinger et al., 2003). The compound 2-(1'H-indole-3'-carbonyl)-thiaxole-4-carboxylic acid methyl ester (ITE) was isolated from mouse tissue and found to act as a potent AHR agonist in cell extracts, cultured cells, and in vivo animal models as evidenced by AHR binding, CYP1A1 protein induction and xenobiotic response element (XRE) reporter activity (Henry et al., 2006). While investigating the role of AHR in shear stress and vascular physiology, low density lipoprotein (LDL) was found to increase AHR signaling due largely to lipoprotein modification (McMillan and Bradfield, 2007a). The relative contribution of either of these ligands to AHR function in vivo remains to be elucidated. However, it is likely that AHR signaling in vivo is regulated by endogenous ligands.

Genetic polymorphisms in murine strains aided the discovery and characterization of the $A H R$ gene. In mice, four distinct $A H R$ alleles, $A H^{b-1}, A H^{b-}$ ${ }^{2}, A H^{\mathrm{b}-3}$, and $A H^{\mathrm{d}}$ have been characterized (Poland et al., 1994). C57BL/6J mice express $A H^{b-1}$ which encodes for the amino acid alanine at codon 375 resulting in a 10 -fold higher ligand binding affinity $\left(K_{d}=6-10 \mathrm{pM}\right)$ compared to DBA/2 mice $\left(\mathrm{K}_{\mathrm{d}}=37 \mathrm{pM}\right)$ that express the $A H^{\mathrm{d}}$ allele containing valine at codon 375 (Okey et al., 1989). Accordingly, the congenic strain D2NAhr ${ }^{d}$ was developed and contains the exact genetic background as the C57BL/6J strain except for expression of the $A H R^{d}$ allele. While polymorphisms in mouse AHR typically 
occur in the ligand binding domain, human polymorphisms manifest in the transactivation domain at codons 517, 554, and 570 (Harper et al., 2002). As in mice, human populations express AHR variants that can vary 10 -fold in TCDD binding affinity. However, the mechanisms for this occurrence do not involve the 517, 554, and 570 codon polymorphic variants (Harper et al., 2002; Nebert et al., 2004). The combination of 517 and 554 polymorphisms results in AHR protein that binds TCDD and DNA, but does not sustain CYP1A1 induction (Wong et al. 2001a; Wong et al., 2001b). It is thought that expression of the lower affinity AHR, similar to the protein product from the murine $A H R^{d}$ allele, protects the majority of the human population from the toxic effects of PAHs (Harper et al., 2002). With the abundance of environmental agents able to activate AHR, it is crucial to determine the exact nature of human AHR allelic variation and the resulting consequences on $A H R$ function.

The bulk of AHR studies have focused on modulation and/or participation in the response to and clearance of xenobiotic compounds. Evidence continues to mount implicating AHR in proliferation, development, adhesion and migration, and proteasomal degradation of steroid hormone receptors (for reviews see (Barouki et al., 2007; Ohtake et al., 2007)). Studies using AHR -/- mice support a scenario in which the AHR acts as an important nuclear transcription factor during embryogenesis and throughout maturity in multiple organisms. AHR-/mice exhibit deficits in vascular structures stemming from a failure of the ductus venosus to close and result in reduced liver weight, microvesicular fatty 
metamorphosis, prolonged extramedullary hematopoeisis, and portal hypercellularity (Fernandez-Salguero et al., 1995; Lahvis et al., 2000; Lahvis et al., 2005; Schmidt et al., 1996). Our lab has shown that AHR-null mice exhibit delayed nephrogenesis resulting in compromised renal development as evidenced by nephropenia and decreases in renal vasculature (Falahatpisheh and Ramos, 2003; McMillan and Bradfield, 2007b). We have also shown that these changes can be reproduced by downregulation of AHR protein upon ligand binding

Of direct relevance to studies of renal function are experiments in which AHR -/- mice were found to have cardiac hypertrophy and elevated mean arterial pressure (Lund et al., 2003; Lund et al., 2006). Hypertension correlated to increased in endothelin-1 and angiotensin II, known regulators of cardiomyocyte hypertrophy (Gavras and Gavras, 2002; Yamazaki et al., 1996). Angiotensin II is a potent renal vasoconstrictor that constricts efferent arterioles and increases blood pressure. Thus, it is possible that hypertension in AHR -/- mice is caused by compromised renal development; although this hypothesis remains to be tested. More studies are required to determine how environmental PAH exposure affects AHR protein expression and function in the context of organogenesis.

As noted previously, PAHs are compounds formed during the incomplete burning of organic substances such as gas, oil, wood, tobacco, and charbroiled 
meat. The primary source of exposure occurs through inhalation of compounds via tobacco smoke, wood smoke, ambient air, and dietary intake. PAHs like BaP are highly lipophilic chemicals that move across cell membranes with relative ease. Upon entry into the cells they associate with hydrophobic molecules and are dispersed throughout the cell accumulating in the mitochondria and nucleus (Barhoumi et al., 2000; Zhu et al., 1995). Once in the bloodstream they bind albumin and lipoproteins and are rapidly distributed throughout the body (for review see (Miller and Ramos, 2001b)). Average PAH exposure can range from 3 to $15 \mu \mathrm{g} /$ day depending on dietary intake. Humans exposed to active or passive smoke can receive as much as $30 \mu \mathrm{g}$ /day (Menzie et al., 1992a; Menzie et al., 1992b; Santodonato et al., 1981).

In mouse models of inhalation exposure, $\mathrm{BaP}$ bioaccumulates primarily in the kidney and to a lesser extent in the liver (Mitchell, 1982). Consistent with the known expression of XMEs, elimination in the liver occurs at 12 hours postexposure while significant amounts of BaP are still present in the kidneys for up to 48 hours (Mitchell, 1982). In utero exposure with radiolabeled BaP show that $\mathrm{BaP}$ enters the fetus and activates classical responses triggered by $\mathrm{PAH}$ metabolism including increases in CYP450 and BaP hydroxylase expression (Neubert and Tapken, 1988a; Neubert and Tapken, 1988b).

$\mathrm{PAH}$ exposure is known to be a causative factor in carcinogenesis, atherogenesis, and embryotoxicity (for review see (Miller and Ramos, 2001b)). 
In mice, metabolism of $\mathrm{BaP}$ into compounds that give rise to DNA adduct formation results in tumor formation in both skin and systemic models. Intraperitoneal injections of the 7,8-diol-9,10-epoxide (BPDE) metabolite induces systemic malignant lymphoma as a result of DNA mutagenesis (Kapitulnik et al., 1977). PAHs affect growth and differentiation of aortic smooth muscle cells and may participate in atherogenesis (Holderman et al., 2000; Kerzee and Ramos, 2000; Ramos and Parrish, 1995). The mechanistic causalities of PAH-induced diseases are complicated by the variety of effects they exert such as DNA mutagenesis, oxidative stress, protein oxidation, and modifications of signal transduction pathways.

$\mathrm{PAH}$ exposure during critical windows of fetal development exhibit teratogenic and embryotoxic effects (Lummus and Henningsen, 1995; MacKenzie and Angevine, 1981a; Rodriquez et al., 1999; Wells et al., 1997; Winn and Wells, 1997). Murine in utero exposure of $150 \mathrm{mg} / \mathrm{kg} \mathrm{BaP}$ causes $\mathrm{T}$ cell deficiencies in offspring (Lummus and Henningsen, 1995). In utero exposure to as little as $10 \mu \mathrm{g} / \mathrm{kg}$ results in reproductive deficits in both male and female offspring manifest by a reduction in the size of seminiferous tubules and corpus lutea deficiencies, respectively (Mackenzie and Angevine, 1981b).

Of relevance to our work is the extensive evidence linking maternal PAH exposure to fetal deficits. Fetal tobacco syndrome is characterized by maternal exposure to PAHs in the form of tobacco smoke and results in reductions in birth 
weight, height, thoracic circumference (for review see (Nieburg et al., 1985)). Evidence supports the theory that active smoking morphologically alters the placenta compromising fetal capillary function resulting in a reduction of oxygen flow to the developing fetus (Bush et al., 2000a; Bush et al., 2000b). Sasaki et al. has provided the first in vivo human evidence that AHR, CYP1A1, and GST1 genetic polymorphisms influence fetal health upon maternal tobacco smoke exposure. Expression of AHR protein with arg/arg at codon 211 significantly lowered birth weight and length. Interestingly, variants of enzymes regulated by AHR activity (CYP1A1 m1/m2 + m2/m2) or involved in PAH elimination (GSTM1I-) sensitized fetuses to the effects of maternal smoking (Sasaki et al., 2006). This study directly links PAH exposure to deficits in AHR signaling in the context of fetal programming.

Fetal programming is a process whereby a stimulus at a sensitive critical period of development induces lasting effects on the structure or function of the organism (Barker, 2004). Some of the strongest evidence of fetal programming involves the correlation between low birth weight (LBW) and compromised renal development. Chronic kidney disease (CKD) affects greater than 20 million people in the U.S.. It is thought that the two major causes are hypertension (HTN) and diabetes mellitus. The kidney is a key regulator of HTN. Most genetic mutations associated with HTN involve proteins expressed in the kidney and regulated renal function such as epithelial $\mathrm{Na}+$ channel (Lifton et al., 2001). This 
led Brenner et al. to postulate that HTN may be associated with congenital deficits in nephron number resulting in impaired renal function.

In humans, a direct correlation exists between birth weight and nephron number. LBW humans have been shown to have reduced nephron numbers and glomerular hypertrophy. An increase in $1 \mathrm{~kg}$ of birth weight results in the addition of 250,000 nephrons (Hoy et al., 2003; Hughson et al., 2003). LBW adults with low nephron number are prone to microalbuminuria, proteinuria, and decreased glomerular filtration (Celsi et al., 1998; Nwagwu et al., 2000; Sanders et al., 2005). Thus, it is likely that intrauterine factors, including PAHs, compromise renal development resulting in low nephron number. The reduced renal capacity may result in glomerular hypertension and subsequent compensatory hypertrophy that in turn leads to disruption of the glomerular basement membrane manifest as glomerulosclerosis. Continued glomerular injury may lead to increases in nephron loss further reducing renal capacity as evidenced by decreases in $\mathrm{Na}^{+}$excretion and glomerular filtration rates. As depicted in Figure 9, this culminates in HTN exacerbating glomerulosclerosis, nephron loss, and proceeds until progressive renal failure.

The work completed as part of this dissertation builds upon interesting studies completed in the Ramos laboratory for the past 10 years establishing a direct link between AHR and cleficits in renal cell differentiation (Alejandro et al., 2000) and post-transcriptional regulation of WT1 isoform expression 
Figure 9. Proposed mechanism of fetal programming in hypertension, renal disease, and glomerular filtration. Intrauterine factors, including PAHs, compromise renal development resulting in low nephron number (Celsi et al., 1998; Nwagwu et al., 2000; Sanders et al., 2005). Reduced renal capacity results in glomerular hypertension and subsequent compensatory hypertrophy that in turn leads to disruption of the glomerular basement membrane manifested as glomerulosclerosis. Continued glomerular injury leads to increases in nephron loss further reducing renal capacity as evidenced by decreases in $\mathrm{Na}^{+}$ excretion and glomerular filtration rates. This culminates in HTN exacerbating glomerulosclerosis, nephron loss, and proceeds until progressive renal failure (Zandi-Nejad et al., 2006). 


\section{Figure 9}

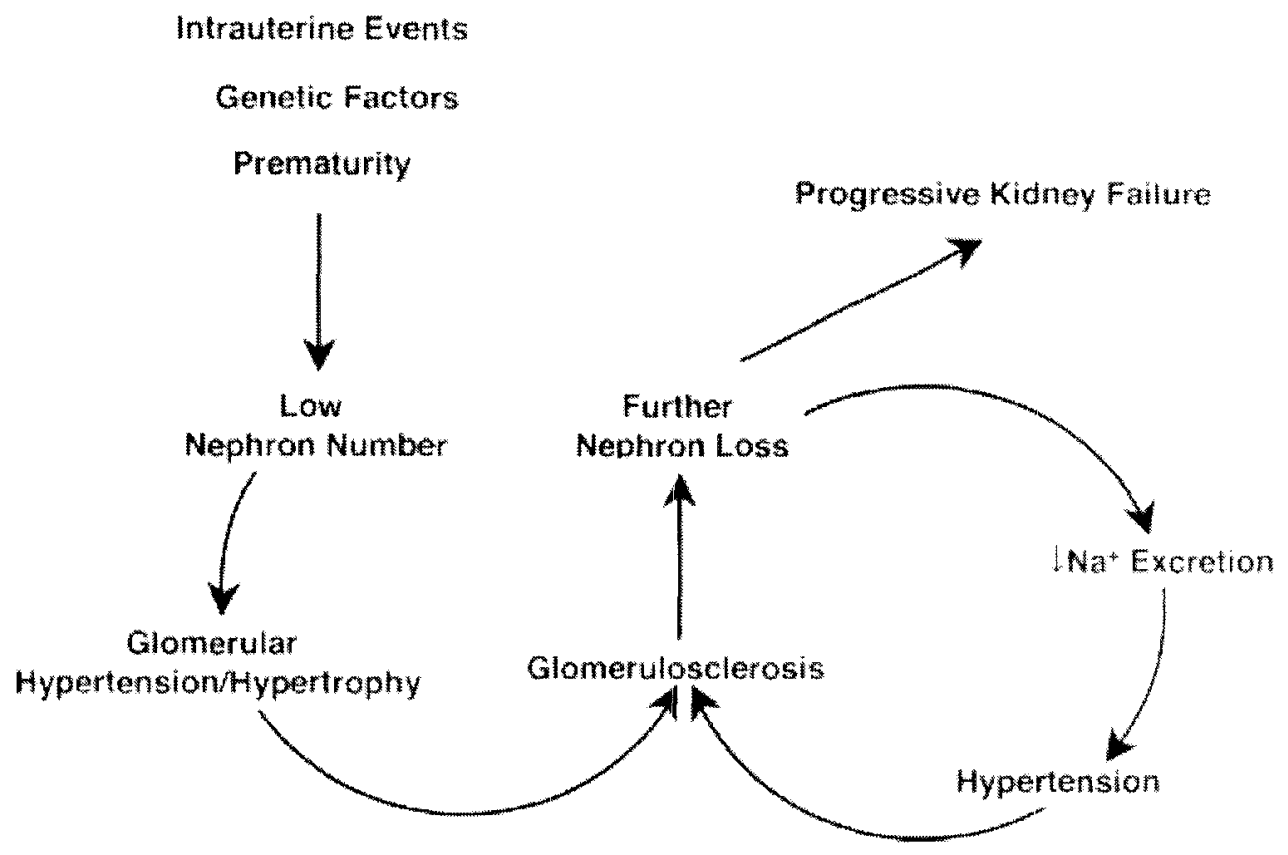


(Falahatpisheh and Ramos, 2003). Using a metanephric kidney organ culture system, we have shown that exposure to $\mathrm{BaP}$, a hydrocarbon ligand for the AHR, inhibits nephrogenesis and increases -KTS/+KTS WT1 mRNA ratios. Hydrocarbon challenge of metanephroi extracted from AHR knockout mice are protected from $\mathrm{BaP}$-induced deficits in nephrogenesis and express higher amounts of +KTS mRNA, indicating that AHR is required in nephrogenesis.

The overall goal of the research summarized in this dissertation was to elucidate the role of AHR in the regulation of WT1 function during nephrogenesis. Specifically, studies were conducted to test the hypothesis that ligand-activated AHR regulates murine nephrogenesis in vivo by altering expression of renal cell differentiation genes through modulation of WT1 mRNA splicing. This hypothesis was tested by three specific aims designed to:

1) Determine whether in utero BaP exposure causes $A H R$ dependent inhibition of nephrogenesis and alterations in WT1 mRNA splicing;

2) Elucidate the time-related effects of AHR ligands on nephrogenesis and WT1 splicing, and;

3) Evaluate the influence of alterations in WT1 splicing on downstream WT1 targets and cell differentiation.

In chapter 2, we evaluate the impact of in utero BaP exposure on nephrogenesis and WT1 mRNA splicing. Previous work has established that 
exposure of metanephric kidney cultures (isolated from C57BL/6J wild type mice) for 4 days to BaP results in inhibition of nephrogenesis and modulation of WT1 mRNA variants. AHR-null metanephroi exhibit similar deficits in nephrogenesis in the absence of BaP exposure (Falahatpisheh and Ramos, 2003). While this regimen was adequate for initial studies, little was known about the effects of in utero BaP exposure on nephrogenesis and WT1 mRNA splicing in vivo. Thus, studies were designed to evaluate the impact of $\mathrm{BaP}$ treatment on pregnant C57BL/6J and D2NAHR ${ }^{d}$ mice expressing the responsive and nonresponsive AHR alleles, respectively. Of particular relevance was the fact that our studies examined concentrations of $\mathrm{BaP}$ known to be relevant to human exposures to the hydrocarbon. We hypothesized that in utero BaP exposure results in AHR mediated inhibition of renal development and alterations in WT1 mRNA splicing.

Chapter 3 summarizes the results of studies completed to define the kinetic profiles of BaP-induced inhibition of nephrogenesis and alteration of WT1 splicing. Experiments focused on defining the mechanism for AHR participation in renal developmental control. Previous work established that exposure of metanephric kidney cultures to BaP for 4 days results in inhibition of nephrogenic differentiation and modulation of WT1 isoforms regulated by AHR. While this work implicated a relationship between AHR and WT1, the kinetic profiles of AHR activation, changes in WT1 mRNA splicing, and inhibition of nephrogenic inhibition had not been defined. Thus, experiments were conducted to determine the extent to which sub-chronic BaP exposure induces nephrogenic inhibition 
and alterations in AHR-WT1 signaling. Through the use of pharmacological AHR antagonists and D2NAHR ${ }^{d}$ metanephroi we examined the molecular mechanism by which PAHs affect nephrogenesis. We hypothesized that BaP-induced disruption of AHR signaling downregulates renal cell differentiation through a pathway that involves altered conservation of WT1 mRNA splice variant ratios.

Chapter 4 focuses on the influence of alterations in WT1 splicing on downstream WT1 targets and cell differentiation. The key event in nephrogenesis is the transdifferentiation of mesenchymal cells to epithelial cells. Recent work has shown that degradation of AHR via ligand-activated ubiquitination or siRNA interference induces the +17 aa and - KTS splice variants of the Wt1 gene (Falahatpisheh and Ramos, 2003; Falahatpisheh and Ramos, 2005). Different WT1 isoforms are believed to promote and/or repress different genetic targets (Hosono et al., 1999; Reddy and Licht, 1996), suggesting that epithelial conversion is differentially influenced by different WT1 splice variants. The concept that different WT1 splice variants regulate different functions is consistent with the notion that translation of -KTS mRNA produces a protein product that localizes to the nucleus, whereas the +KTS protein displays preferential cytoplasmic localization. Murine kidney cell lines at two different stages of cellular differentiation were used to determine the role of alterations in WT1 splicing in the regulation of renal cell differentiation. Subsequent experiments were conducted to determine if alterations in various WT1 isoforms lead to induction/repression of proteins directly regulated by WT1 and involved in 
metanephric development. We hypothesized that overexpression of -KTS or the +17aa WT1 isoforms compromises differentiation of mK4, but not mK3 cell lines and selectively modulate downstream targets of WT1. 


\section{CHAPTER 2}

\section{DEREGULATION OF FETAL PROGRAMMING IN THE DEVELOPING KIDNEY BY BENZO(A)PYRENE}

\section{Summary}

Polycyclic aromatic hydrocarbons (PAHs) are environmentally ubiquitous chemicals identified as potential disruptors of developmental programming and inducers of transformed phenotypes in several organ systems. Recent studies in this laboratory have established that exposure of developing kidneys in vitro to $\mathrm{BaP}$, a prototypical $\mathrm{PAH}$, causes deficits in renal cell differentiation and ablates nephrogenesis. The present studies were conducted to determine if similar changes are seen in vivo following in utero exposure to $\mathrm{BaP}$ at concentrations relevant to those likely to be encountered by humans during environmental exposures. Pregnant C57BL/6J (C57) and B6.D2N-Ahr/J (D2N) mice expressing the wild type AHR allele or an AHR allele with reduced ligand binding affinity, respectively, were treated by gavage with 0.1 or $0.5 \mathrm{mg} / \mathrm{kg} \mathrm{BaP}$ daily beginning 10 days post coitum (dpc) through 13 . This exposure regimen was 
designed to mimic human exposure levels during periods of nephrogenesis. Pregnant mice were allowed to come to term and offspring were examined at 7 days and at 12 months post partum for indicators of renal morphology and measurements of renal function. Exposure to $0.1 \mathrm{mg} / \mathrm{kg}$ and $0.5 \mathrm{mg} / \mathrm{kg} \mathrm{BaP}$ caused AHR allele-specific decreases in glomerular number and altered renal morphology compared to vehicle-treated controls. Developmental deficits were primarily of glomerular origin, as evidenced by decreased podocyte numbers and increased levels of urinary albumin, a marker of glomerular function. Markers for collecting duct and distal tubule injury, namely, rat papillary antigen 1 (RPA1) and glutathione S-transferase Yb1 (GSTYb1), were unchanged. Both morphological and molecular endpoints correlated with the loss of the AHR protein in kidneys from BaP-exposed mice. These results implicate $\mathrm{BaP}$ as an influential factor in fetal programming during the course of murine renal development.

\section{Introduction}

Fetal programming is a process whereby a stimulus at a sensitive critical window of development induces lasting effects on the structure or function of the organism (Barker, 2004). Correlations between low birth weight (LBW) and renal development have provided some of the strongest evidence for environmental disruption of fetal programming. LBW humans have reduced nephron numbers and glomerular hypertrophy. In humans, an increase of $1 \mathrm{~kg}$ in birth weight results in the addition of 250,000 nephrons (Hoy et al, 2003; Hughson et al., 
2003). LBW adults with low nephron number are prone to microalbuminuria, proteinuria, and decreased glomerular filtration (Celsi et al., 1998; Nwagwu et al. 2000; Sanders et al., 2005). Reduced renal capacity results in glomerular hypertension and subsequent compensatory hypertrophy that in turn leads to disruption of the glomerular basement membrane manifested as glomerulosclerosis. Sustained glomerular injury exacerbates nephron loss further reducing renal glomerular filtration rate. A reduction in renal capacity further increases blood pressure and completes a futile cycle of glomerulosclerosis and nephron loss that may ultimately result in progressive renal failure (Zandi-Nejad et al., 2006). Thus, it is important to examine the mechanisms by which intrauterine factors disrupt ontogenesis.

PAHs are compounds formed during the incomplete burning of organic substances such as gas, oil, wood, tobacco, and charbroiled meat. Routes of exposure include inhalation via tobacco smoke, wood smoke, ambient air, as well as dietary intake (USDHHS, 1995). PAH exposure is a known causative factor in carcinogenesis, atherogenesis, and embryotoxicity and these effects are believed to be mediated by mechanisms involving DNA mutagenesis, oxidative stress, protein oxidation, and deficits in signal transduction (for review see (Miller and Ramos, 2001b)). PAHs acting as ligands of nuclear transcription factors such as aryl hydrocarbon receptor (AHR) can induce deficits in embryogenesis via disruption of constitutive AHR signaling (Falahatpisheh and Ramos, 2003; Nanez and Ramos, 2007; Peters et al., 1999a). 
AHR is a basic helix-loop-helix transcription factor activated by endogenous and exogenous ligands including PAHs. Upon ligand binding, AHR disassociates from two molecules of the chaperone heat shock protein 90 (HSP90), binds the X-associated protein 2 (XAP2) and p32. The resulting complex exposes a nuclear localization sequence that facilitates nuclear translocation. This complex in turn binds to the aryl hydrocarbon receptor nuclear translocator (ARNT or HIF1 $\beta$ ) in the nucleus resulting in DNA binding to sequences containing 5'-GCGGGGGCG-3' and (TCC) $n$ repeats (Denison and Nagy, 2003). Translocation of the AHR-ligand complex to the nucleus triggers its proteolytic degradation regardless of ARNT binding (Roberts and Whitelaw, 1999). Depending upon cell type, AHR can be regulated through negative feedback inhibition of AHR transcriptional activation by the AH repressor protein (AHRR) (Mimura et al., 1999).

In mice, four distinct $A H R$ alleles, $A H^{\mathrm{b}-1}, A H^{\mathrm{b}-2}, A H^{\mathrm{b}-3}$, and $A H^{\mathrm{d}}$ have been characterized (Poland et al., 1994). C57BL/6J mice express $A H^{b-1}$ which encodes for the amino acid alanine at codon 375 and results in a 5-fold higher ligand binding affinity $\left(K_{d}=6-10 \mathrm{pM}\right)$ compared to $\mathrm{DBA} / 2$ mice $\left(\mathrm{K}_{d}=37 \mathrm{pM}\right)$ which express the $A H R^{\mathrm{d}}$ allele containing valine at codon 375 (Okey et al., 1989). The ala-val substitution results in a conformational change that substantially decreases ligand binding affinity without affecting association with HSP90. This renders the $A \mathrm{HR}^{\mathrm{d}}$ allele functionally null as exposure to ligand does not promote nuclear translocation, association with ARNT, AHRRE transcriptional activation, 
or proteasomal degradation when compared to $\mathrm{AHR}^{\mathrm{b}}$ (Poland and Glover, 1990; Poland et al., 1994). Accordingly, the congenic strain D2NA.hr ${ }^{d}$ was developed to contain the exact genetic background as the $\mathrm{C} 57 \mathrm{BL} / 6 \mathrm{~J}$ strain except for expression of the $A H R^{\mathrm{d}}$ allele (Nebert et al., 1972). Humans express AHR genetic polymorphic variants that alter AHR function. Relevant to our studies is that expression of AHR protein with arg/arg at codon 211 significantly lowered birth weight and length in offspring exposed to in utero tobacco smoke (Sasaki et al., 2006). Interestingly, variants of enzymes regulated by AHR activity (CYP1A1 $\mathrm{m} 1 / \mathrm{m} 2+\mathrm{m} 2 / \mathrm{m} 2$ ) or involved in PAH elimination (GSTM1 - /-) sensitized fetuses to the effects of maternal smoking (Sasaki et al., 2006). This study directly links $\mathrm{PAH}$ exposure to deficits in AHR signaling in the context of fetal programming.

Evidence continues to mount implicating AHR in the regulation of proliferation, development, adhesion and migration, and proteasomal degradation (for reviews see (Barouki et al., 2007; Ohtake et al., 2007)). Based on previous findings in our laboratory, as well as others, we hypothesized that AHR acts as an important nuclear transcription factor during embryogenesis and throughout maturity (Ramos et. al., 2007). AHR-/- mice exhibit deficits in vascular structures stemming from a failure of the ductus venosus to close and result in reduced liver weight, microvesicular fatty metamorphosis, prolonged extramedullary hematopoeisis, and portal hypercellularity (Fernandez-Salguero et al., 1995; Lahvis et al., 2000; Lahvis et al., 2005; Schmidt et al., 1996). Our laboratory has shown that AHR-null mice exhibit delayed nephrogenesis resulting 
in compromised renal development as evidenced by nephropenia and decreases in renal vasculature (Falahatpisheh and Ramos, 2003; McMillan and Bradfield, 2007b). These changes can be reproduced by decreased AHR protein levels upon ligand binding (Nanez and Ramos, 2007). In addition, AHR -/- mice were found to have endothelin1 and angiotensin II mediated cardiac hypertrophy and elevated mean arterial pressure, conditions directly linked to impaired renal functions (Gavras and Gavras, 2002; Lund et al., 2003; Lund et al., 2006; Yamazaki et al., 1996).

Using a metanephric kidney organ culture system, our laboratory has shown that $\mathrm{BaP}$ exposure inhibits nephrogenesis as evidenced by decreases in branching morphogenesis, glomerular number, and decreases in undifferentiated cells. BaP-induced developmental deficits correlate with downregulation of renal cell differentiation markers and expression of the AHR. Here, we test the hypothesis that in utero exposure to $\mathrm{BaP}$ inhibits nephrogenesis through a mechanism involving ligand-mediated disruption of AHR signaling. As an evolving role for AHR in developmental biology continues to unfold, this work will help to determine the mechanisms by which disruption of AHR signaling alters fetal programming within the context of exposure to environmental chemicals.

\section{Methods}




\section{In utero BaP exposure}

Time-pregnant C57BL/6J and B.6 D2N-AHR ${ }^{d / j}$ females were exposed to BaP (Sigma-Aldrich, St. Louis, MO) or medium chain triglyceride oil (MCT) (Mead Johnson Nutritionals, Evansville, $I \mathrm{~N}$ ) at doses of $0.1 \mathrm{mg} / \mathrm{kg}$ and $0.5 \mathrm{mg} / \mathrm{kg}, 10$ through 13 d.p.c. by oral gavage. Murine in utero exposure of $150 \mathrm{mg} / \mathrm{kg} \mathrm{BaP}$ is known to cause offspring T cell deficiencies (Lummus and Henningsen, 1995). Thus, the concentrations chosen for study were considerably lower than those established for in utero toxicity and teratogenecity (Lummus and Henningsen, 1995; MacKenzie and Angevine, 1981a; Rodriquez et al., 1999; Wells et al., 1997; Winn and Wells, 1997) and when normalized for species-dependent temporal differences in development, are representative of human exposure of at risk populations (Menzie et al., 1992a; Menzie et al., 1992b; Rebagliato et al., 1995).

Sources of human and animal PAH exposure include air, water, food, and soil and average 6-15 $\mu \mathrm{g} /$ day (Menzie et al., 1992a; Menzie et al., 1992b).

Depending on environment, food is thought to be the largest contributor. Active or passive smoking doubles PAH exposure to $30 \mu \mathrm{g} /$ day (Santodonato et al., 1981). Assuming an average bodyweight of $60 \mathrm{~kg}$, the average human intake is estimated to be $0.25 \mu \mathrm{g} / \mathrm{kg} /$ day to $0.5 \mu \mathrm{g} / \mathrm{kg} /$ day for passive or active tobacco exposure. Allowing for differences in the length of mouse and human gestation, $0.012 \mu \mathrm{g} / \mathrm{kg} / \mathrm{day}$ is estimated as the predicted relevant murine exposure. While the 0.1 and $0.5 \mu \mathrm{g} / \mathrm{kg}$ exposure levels examined in our study are higher than 
those encountered by the general U.S. human population, they are functionally relevant to human polymorphisms affecting $\mathrm{PAH}$ metabolism and AHR signaling (Sasaki et al., 2006; Wong et al., 2001a; Wong et al., 2001b), and considerably lower than PAH levels examined in risk assessment studies of carcinogenicity and teratogenicity (Lummus and Henningsen, 1995; MacKenzie and Angevine, 1981a; Rodriquez et al., 1999; Wells et al., 1997; Winn and Wells, 1997).

Seven days after birth, pups were euthanized and renal tissue was fixed in situ. The heart, liver, testis, and aorta were extracted to assess system-specific effects of in utero BaP exposure. Pups from the same litter were monitored for 12 months and examined for markers of nephropathy.

\section{Glomerular morphometric analysis and Immunohistochemistry}

Resected kidneys were fixed in fresh $4 \%$ paraformaldehyde at $4^{\circ} \mathrm{C}$ for 12 hr and embedded in paraffin. $5 \mu \mathrm{m}$ sections were cut and processed, then stained with hematoxylin and eosin. For immunohistochemical analysis, slides were treated under pressure with Vector Antigen Unmasking Solution $®(V e c t o r$ Laboratories, Burlingame, CA). WT1 (180) (Santa Cruz Biotechnology, Santa Cruz, CA) or AHR rabbit polyclonal (Biomol International, Plymouth Meeting, PA) antibodies were applied overnight at $4{ }^{\circ} \mathrm{C}$ in a solution of $0.3 \%$ Triton $\mathrm{X}-100$ and $5 \%$ goat serum (Vector Laboratories, Burlingame, CA). Primary antibodies were bound to a goat anti-rabbit biotinylated secondary antibody (Invitrogen-Molecular Probes, Carlsbad, CA), amplified with $A B 3$ Celite $\AA$, developed with 
diaminobenzidine (DAB), and counterstained with Mayer's Hematoxylin (Vector Laboratories, Burlingame, CA).

Glomerular numbers were quantified manually and verified by image analysis. For all analyses, images of entire kidney cross sections from 5 renal planes were captured at 20X. Using the Zeiss Axiovision Rel 4.3 image analysis software, images of entire renal cross sections were filtered for intensity, color thresholding, and size of glomerular WT1 IHC signal allowing for distinction between glomeruli and other structures. Values were normalized to renal area in all instances.

WT1 and AHR protein was measured by optimizing thresholding relative to negative control serial sections. Indices of protein expression were expressed as sum density normalized to total renal area. Podocyte number was quantified using WT1 signal filtered for intensity, color thresholding, and size. All values were normalized to glomerular number as measured by image analysis.

Statistical significance was evaluated using SPSS statistical software (SPSS, SPSS Inc., Chicago, IL) and testing for significance between groups by analysis of variance (ANOVA) and least sum of square difference (LSD).

\section{Urinary albumin}


Individual urine samples were collected and stored at $-80^{\circ} \mathrm{C}$ in urine stabilizing buffer (Biotrin International, Dublin, Ireland). Under reducing conditions, $1 \mu$ l of urine sample was loaded on a $4-12 \%$ Bis-Tris NuPage gel (Invitrogen, Carlsbad, CA) and processed for silver staining as per manufacturer's instructions (Invitrogen, Carlsbad, CA). Sum density' values were calibrated to a mouse serum albumin standard curve ranging from 10 to 0.001 $\mu g / \mu \mathrm{l}$.

\section{Urinary Renal Papillary Antigen 1 (RPA1) and Glutathione S-transferase Yb1 (GSTYb1) Measurements}

Enzyme immunoassays for the quantitative measurement of RPA1 and GSTYb1 were performed as per manufacture's instructions (Biotrin International, Dublin, Ireland). Briefly, 96 well microtiter plates were supplied conjugated with anti-RPA1 or GSTYb1 lgG. Urine was diluted $1 / 25$ and equilibrated for $1 \mathrm{hr}$ at room temperature before addition of antibody-enzyme conjugate. After substrate development, absorbance was read at $450 \mathrm{~nm}$ with $630 \mathrm{~nm}$ as a reference. Absorbance was normalized to internal controls and expressed as relative units.

\section{Analysis of WT1 Splice Variants}

Total RNA was extracted using TRIzol ${ }^{\circledR}$ and CDNA was synthesized using SuperScript II (Invitrogen, Carlsbad CA) as per manufacturer's instructions. Quantitative PCR using primers specific for WT1 isoforms was performed to detect differences in the ratio of WT1splice variants ( \pm KTS) and ( $\pm 17 \mathrm{aa})$ 
(Falahatpisheh and Ramos, 2003; Falahatpisheh and Ramos, 2007). Primers were designed using Beacon Designer 5.1 to create amplicons from 150-300 base pairs with an average melting temperature of $58^{\circ} \mathrm{C}$.

\section{Western Blot Analysis of WT1 Protein Isoforms}

Protein was extracted using T PER reagent (Pierce, Rockford, IL) as per manufacturer's directions. Samples were run on $4-12 \%$ Bis-Tris NuPage gels (Invitrogen, Carlsbad, CA) under reducing conditions and transferred to a PVDF membrane where bound protein was detected using WT1(180) rabbit polyclonal antibody (Santa Cruz Biotechnology, Santa Cruz, CA) and a horseradish peroxidase-conjugated secondary antibody. Previous work in the laboratory has shown that differences in \pm 17 aa mRNA splice variants can be resolved at the protein level via gel electrophoresis.

\section{Results}

\section{Fetal BaP exposure alters renal development and function in offspring}

C57 and D2N mice had similar numbers of glomeruli at 7 day old (Figure 1A). In utero exposure to both 0.1 and $0.5 \mathrm{mg} / \mathrm{kg} \mathrm{BaP}$ caused a significant decrease in glomerular numbers in $\mathrm{C} 55$ but not D2N (Figure $1 \mathrm{~A}$ and $\mathrm{B}$ ). Offspring kidneys of BaP-exposed dams exhibited a reduction in glomerular size and increases in undifferentiated cells compared to controls (Figure 1B). D2N mice expressing the low affinity $\mathrm{AHR}^{\mathrm{d}}$ allele were protected from BaP-induced glomerular deficits (Figure $1 \mathrm{~A}$ and $\mathrm{B}$ ). 
Figure 1. BaP inhibits nephrogenesis via an AHR allele specific

mechanism. Kidneys were resected from 7-day old C57 and D2N-AHR ${ }^{d}$ mice exposed to either $0.1,0.5 \mathrm{mg} / \mathrm{kg}$ BaP or MCT oil vehicle in utero. Tissue was fixed in $4 \%$ paraformaldehyde and processed for modified hematoxylin and eosin staining, to visualize differentiated structures. Serial sections were processed for immunohistochemical analysis of WT1 protein and counterstained with hematoxylin. Glomerular number was quantified manually and verified by image analysis. Images of entire kidney cross sections from varying renal planes were captured at 20X. Intensity and color thresholding for glomerular WT1 signal was modified to exclude both small and large artifacts approximating glomerular number then normalized to renal area. Accuracy was controlled for by manual analysis of random images. Statistical significance was calculated by ANOVA and LSD post hoc tests $p<0.05$. Panels $A$ and $B$, show that exposure to BaP decreases glomerular number in C57 mice via an AHR allele-specific mechanism. Scale represents $200 \mu \mathrm{m}$. 
Figure 1

A

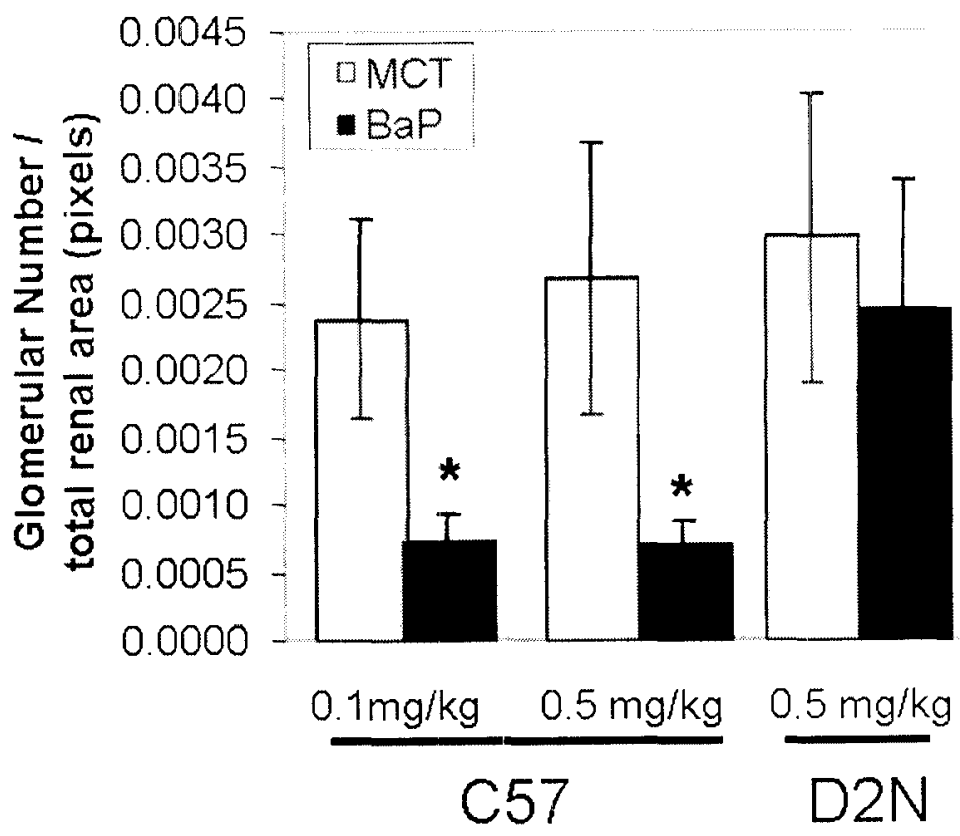


Figure 1

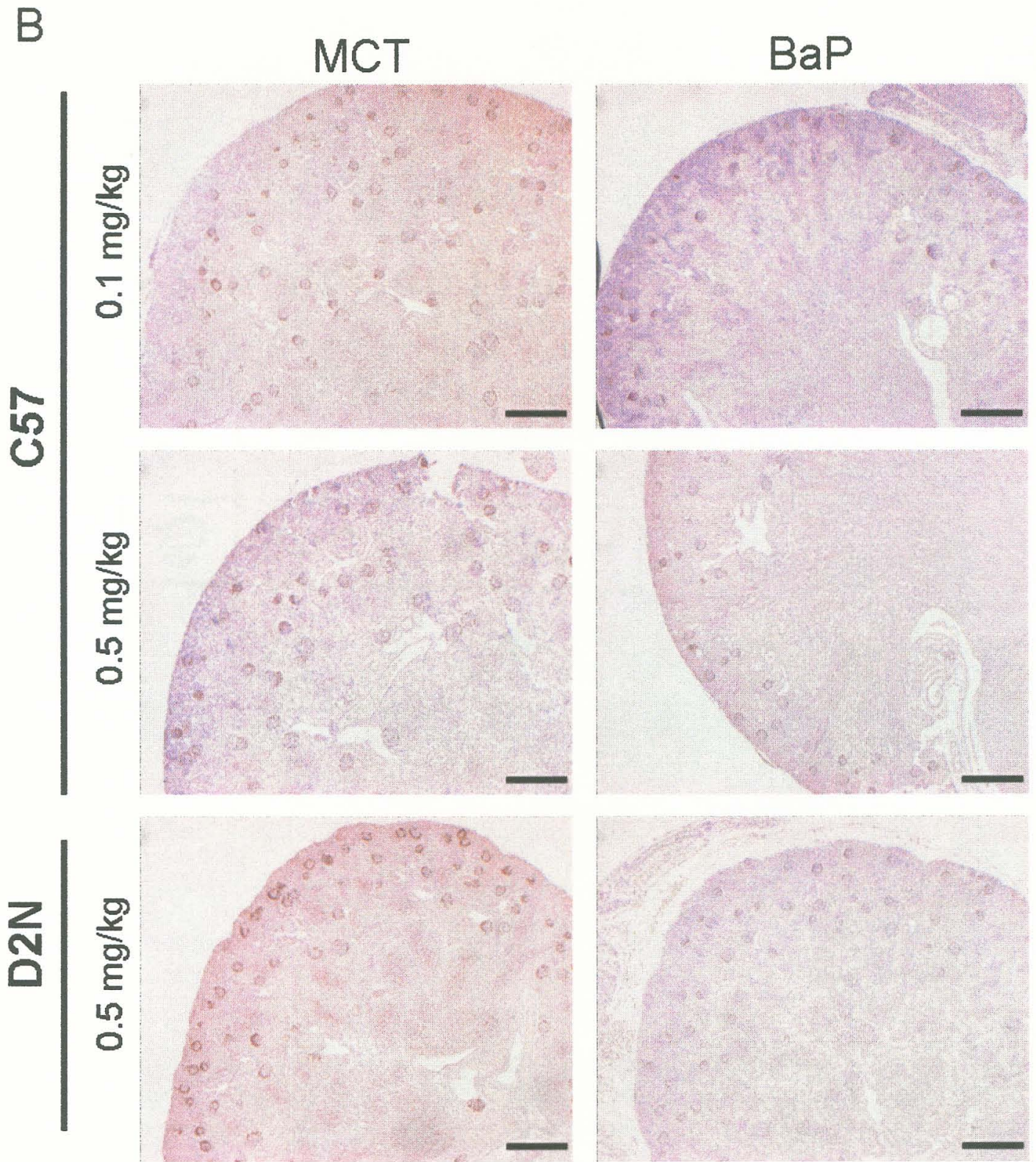


Albuminuria is a hallmark of renal nephropathy and a common clinical marker for the diagnosis of renal disease (Cameron and Blandford, 1966). In agreement with previous work, albumin was the most abundant protein present in mouse urine (Figure 2B) (Marshall and Williams, 1987). C57 and D2N mice excreted similar amounts of urinary albumin demonstrating that expression of the $\mathrm{AHR}^{\mathrm{d}}$ allele does not affect renal development in unstressed animals (Figure 2A). In utero $\mathrm{BaP}$ exposure resulted in dose-dependent increases in albuminuria, at 12 months old, which were dependent upon expression of the responsive $A H R^{b}$ allele. Under these conditions, D2N mice were unaffected (Figure $2 \mathrm{~A}$ and $2 \mathrm{~B}$ ).

\section{Fetal $\mathrm{BaP}$ exposure is associated with specific glomerular deficits}

The podocyte is a key regulator in glomerular basement membrane (GBM) homeostasis by lending support to the glomerular tuft and functioning as the chief filtration barrier (Abrahamson, 1987; Sariola et al., 1984). Podocytes produce the majority of the molecular structures necessary for GBM maintenance (Abrahamson, 1987). Murine podocyte division halts 7 days post birth. Thus, impaired renal development, injury, or degeneration of the podocyte population results in deficits affecting adult renal function specifically impairing glomerular filtration (Mundel and Kriz, 1995). Immunohistochemical quantification of podocyte number revealed no differences between C57 and D2N mice (Figure 2C). Both 0.1 and $0.5 \mathrm{mg} / \mathrm{kg}$ in utero BaP exposure elicited decreases in podocyte numbers (Figure 2C). D2N mice did not display BaP-induced deficits in podocyte number, further suggesting the importance of ligand binding in BaP- 
Figure 2. BaP exposure induces glomerular specific injury. Urine was collected from 12 month old offspring C57 and D2N-AHR ${ }^{d}$ mice exposed to either $0.1,0.5 \mathrm{mg} / \mathrm{kg} \mathrm{BaP}$ or MCT oil in utero. Proteins were separated on a $4-12 \%$ Bis-Tris gel and developed by silver stain. Albumin values were normalized to mouse serum albumin standard curve $(10,1,0.1,0.01$, and $0.001 \mu \mathrm{g} / \mu \mathrm{l})$ and statistical significance was calculated by ANOVA and LSD post hoc tests $p<$ 0.05. Panel A, BaP alters albumin urinary levels in C57 mice via a dosedependent, AHR allele-specific mechanism. Panel B, silver stain visualization of mouse urinary albumin in C57 mice exposed in utero to $0.5 \mathrm{mg} / \mathrm{kg} \mathrm{MCT} \mathrm{oil} \mathrm{or}$ BaP. Panel C, Immunohistochemical analysis of podocyte number was quantified using WT1 signal filtered for intensity, color thresholding and size normalized to glomeruli number. BaP exposure results in AHR allele-specific decreases in podocyte numbers. Panel D, Immunohistochemical analysis of total WT1 signal quantified using WT1 normalized to podocyte number. BaP exposure results in AHR allele-specific decreases in podocyte numbers. 
Figure 2

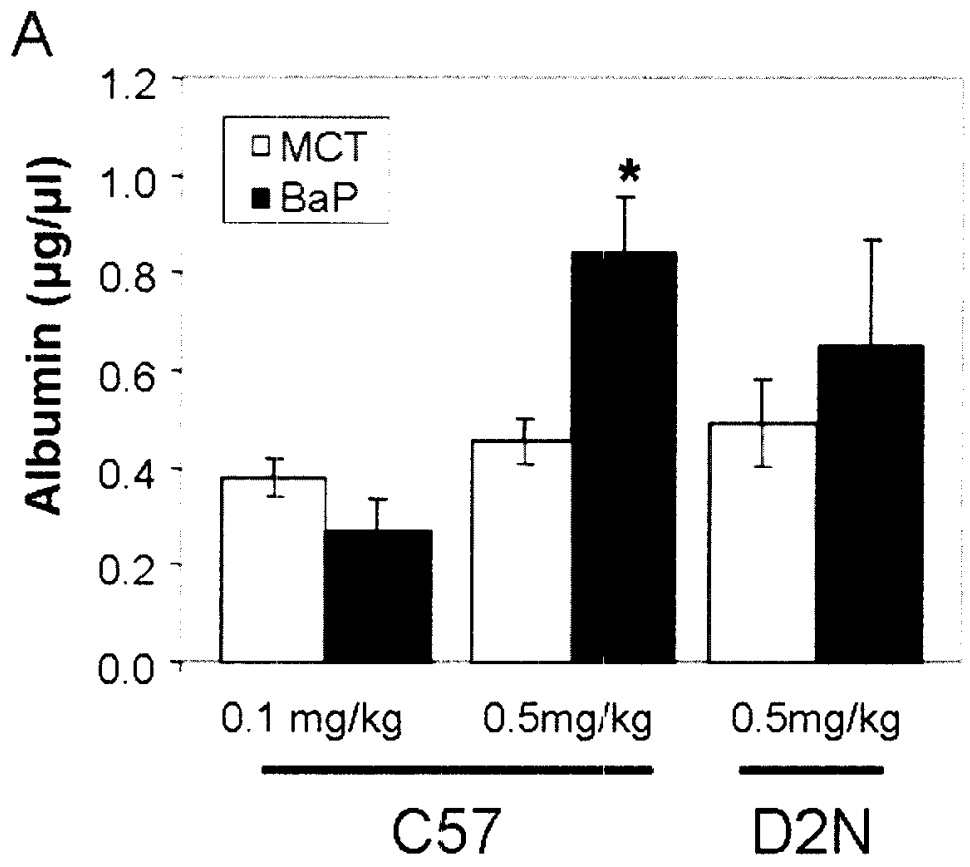

B

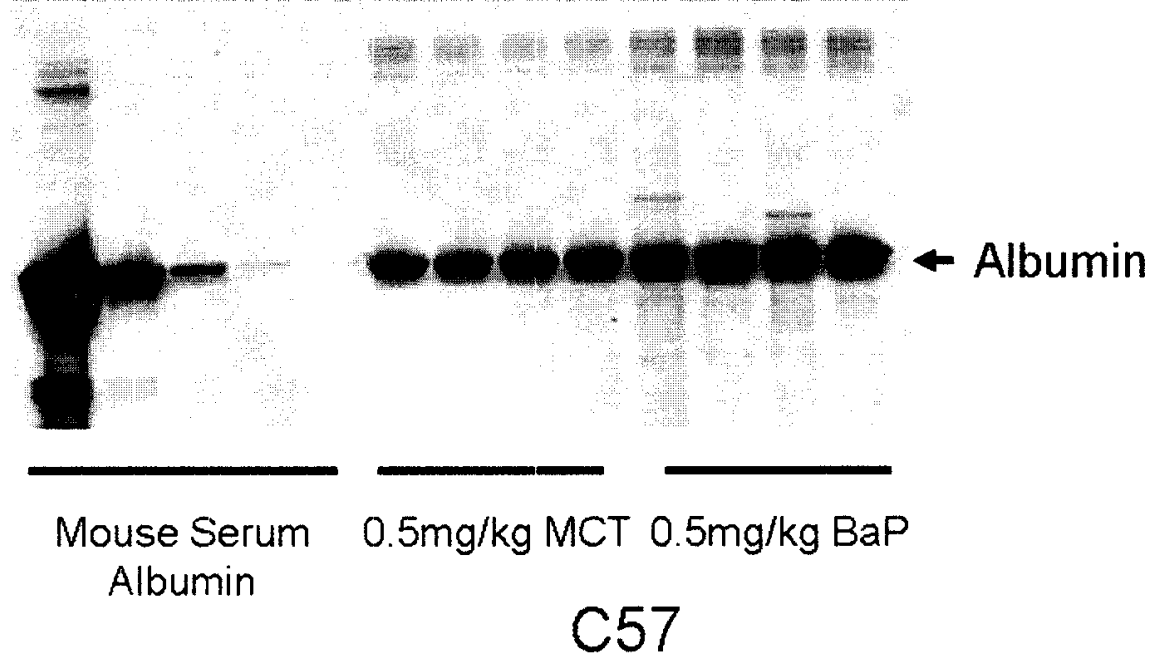


Figure 2

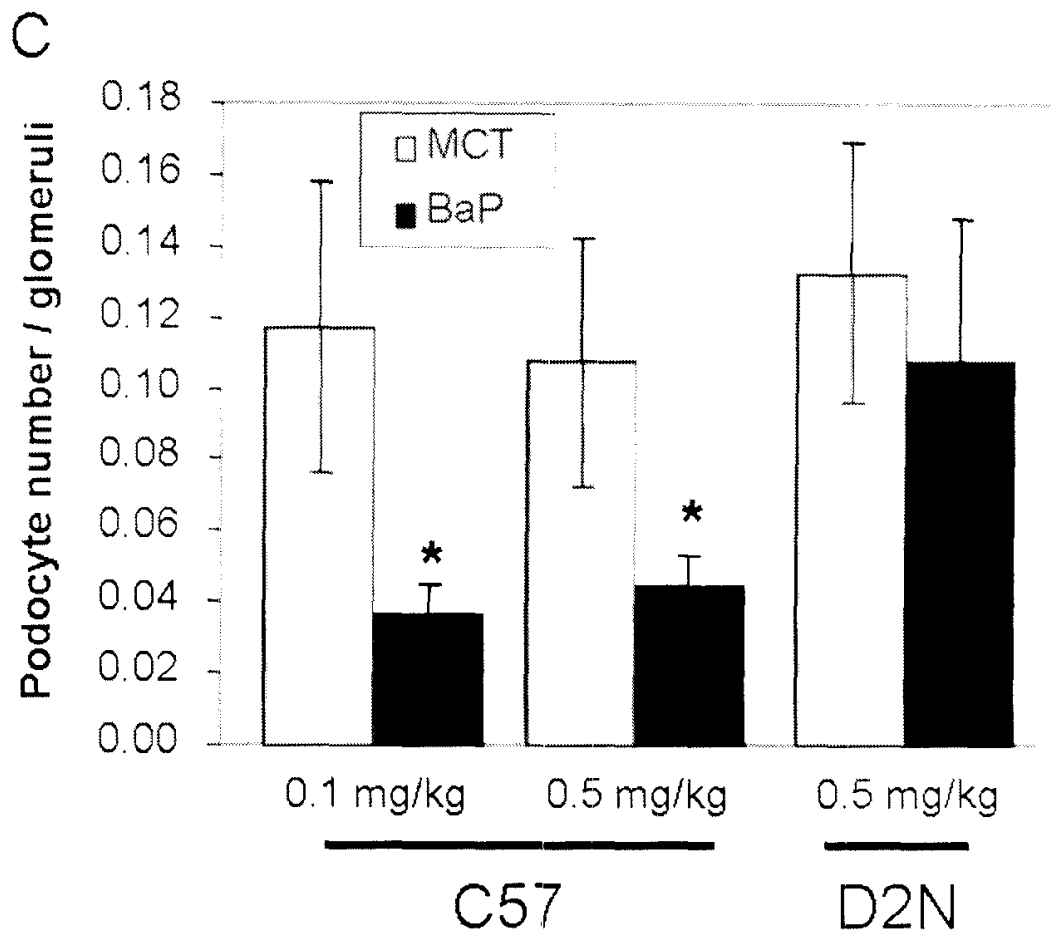

D

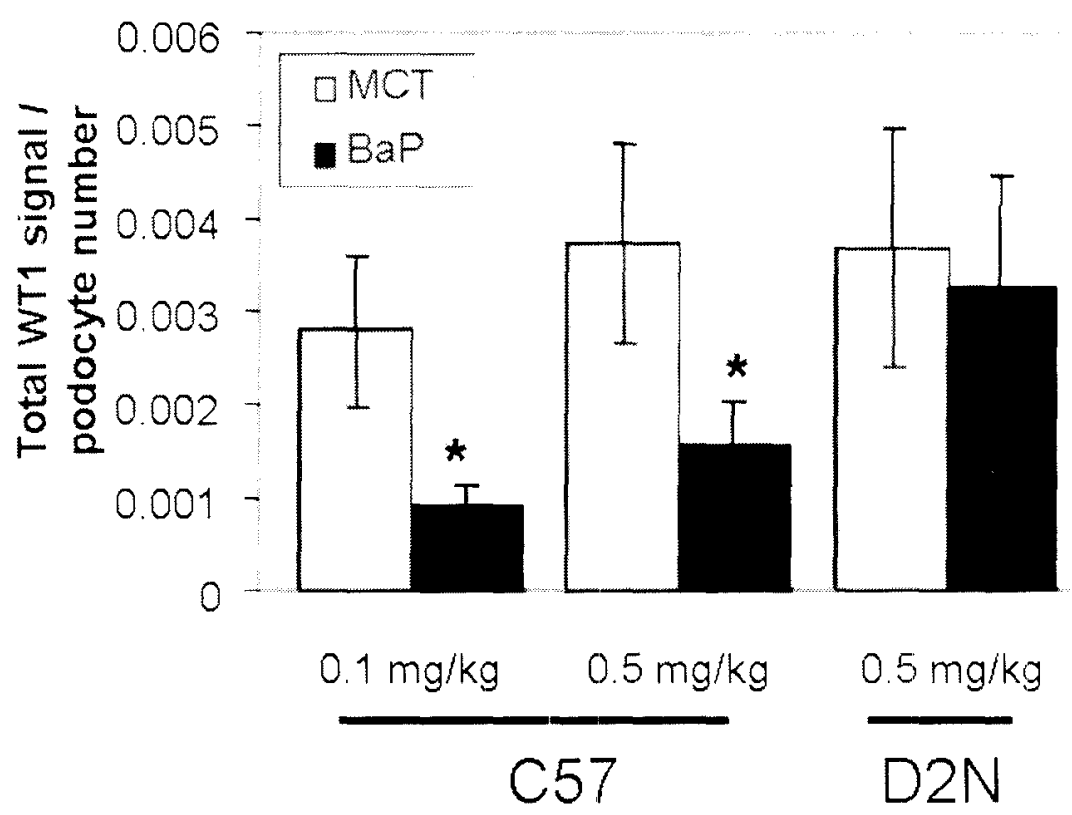


induced deficits in nephrogenesis (Figure 2C). As expected, both 0.1 and 0.5 $\mathrm{mg} / \mathrm{kg}$ in utero BaP exposure decreased WT1 protein expression in an AHR allele specific manner (Figure 2D). WT1 is a known regulator of nephrogenic outcomes in humans and mice (Niaudet and Gubler, 2006). WT1 activity is chiefly regulated in cis by expression of specific mRNA ratios resulting from posttranscriptional modification (Hohenstein and Hastie, 2006; Lee et al., 2002). Ongoing work will examine the effects of in utero BaP exposure on WT1 mRNA splice variant expression in C57 and D2N mice.

Classical markers of nephropathy such as blood urea nitrogen, urinary creatinine, and albuminuria generally manifest in severely compromised kidneys (Mathieson, 2004). A new class of urinary markers has been developed to detect subtle renal injury that precludes gross nephropathy. Rat papillary antigen 1 (RPA1) and glutathione S-transferase Yb1 (GSTYb1) are markers of collecting duct and distal tubule injury, respectively (Falkenberg et al., 1996; Hildebrand et al., 1999; Kilty et al., 1998). RPA1 is specifically produced in the papillary collecting duct and is released into the urine upon exposure to classical renal toxins (Hildebrand et al., 1999). GSTYb1 is highly expressed in the distal and convoluted tubules of the murine renal tubules and is released into the urine upon injury (Eger et al., 1997; Sundberg et al., 1994a; Sundberg et al., 1994b). The low level in utero BaP exposure did not alter urinary RPA1 or GSTYb1 levels in either $\mathrm{C} 57$ or D2N mice (Figure 3). Taken together these findings indicated 
Figure 3. BaP exposure does not induce collecting duct or distal tubular injury. Urine was collected from 12-month old offspring C57 and D2N-AHR mice exposed to either $0.1,0.5 \mathrm{mg} / \mathrm{kg} \mathrm{BaP}$ or MCT oil in utero. Absorbances from enzyme immunoassay detection of RPA1 and GSTYb1 were normalized to internal controls expressed as relative units. Statistical significance was calculated by ANOVA and LSD post hoc tests $p<0.05$. Panels $A$ an $B, B a P$ exposure does not alter relative amounts of RPA1 or GSTYb1 presence in urine. 
Figure 3

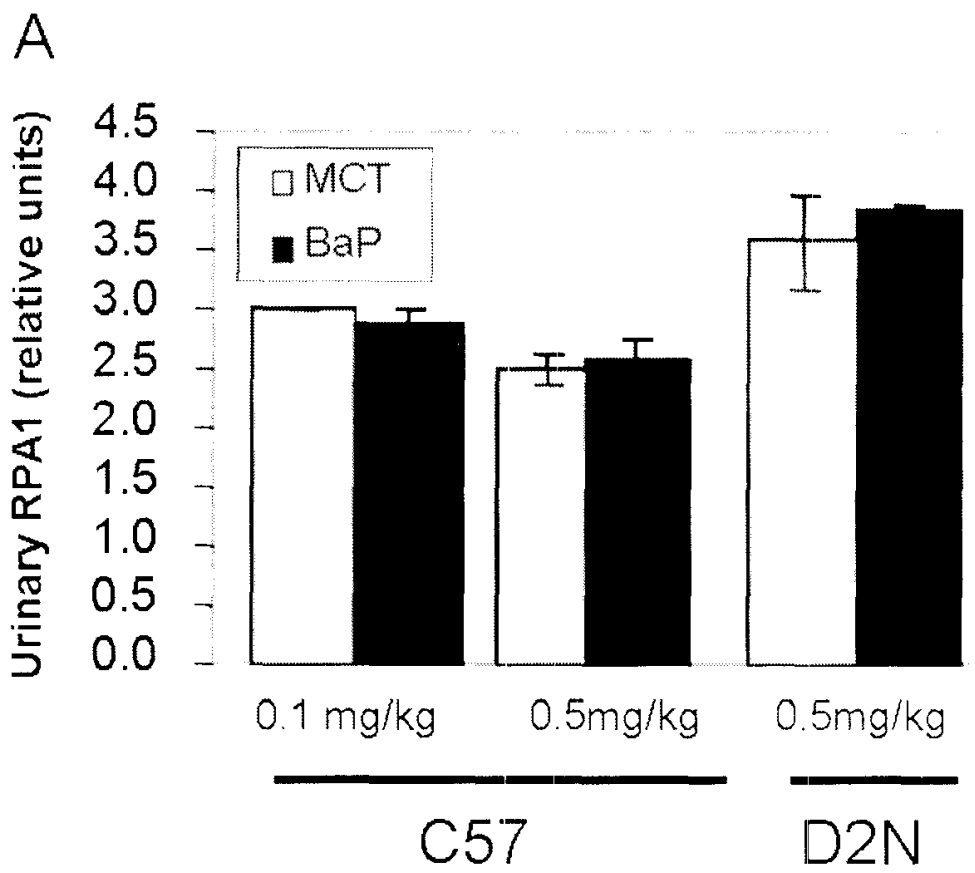

B

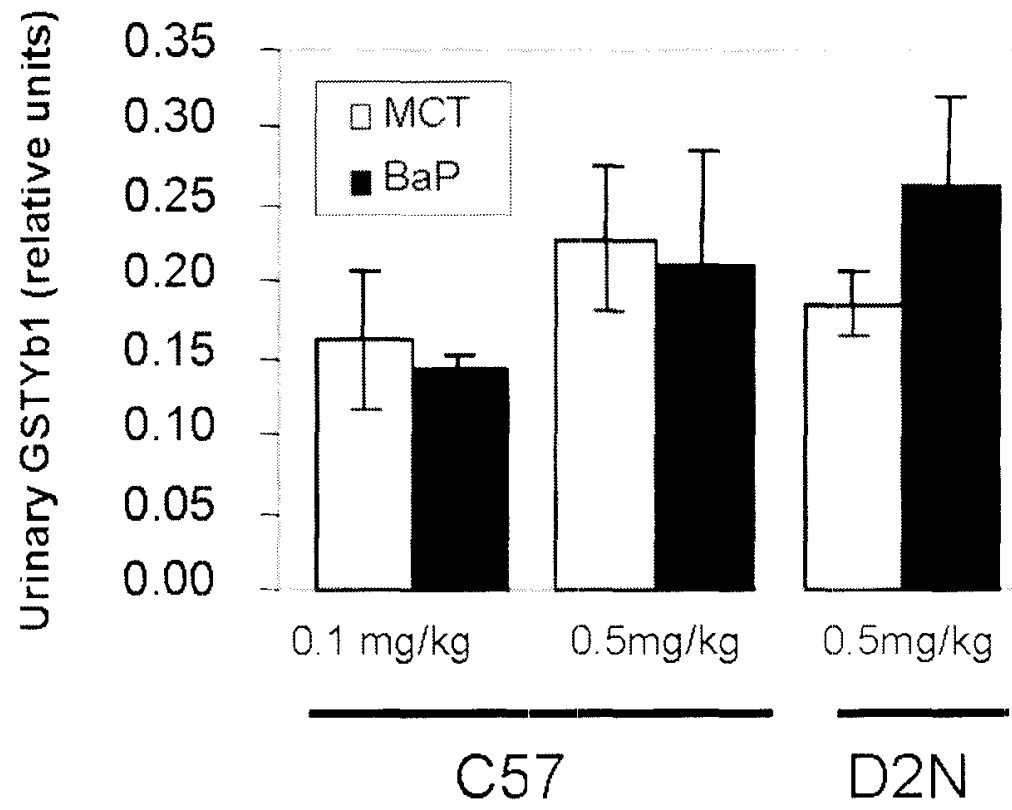


that in utero BaP-exposure results in renal deficits consistent with glomerular nephropathy.

\section{In utero BaP exposure decreases AHR protein expression}

Consistent with known AHR biology, in utero exposure to BaP resulted in dose-dependent decreases in AHR protein in C57 (Figures 4A and B). Loss of receptor protein correlated with AHR variant-specific reductions in glomerular and podocyte number, as well as increased urinary albumin. These results suggest that environmentally relevant in utero $\mathrm{BaP}$ exposures are sufficient to activate AHR and in doing so, promote degradation, likely altering constitutive signaling.

\section{Discussion}

Extensive in utero exposure studies have identified PAHs as potent modulators of fetal development that exert teratogenic and embryotoxic effects, (Lummus and Henningsen, 1995; MacKenzie and Angevine, 1981a; Rodriquez et al., 1999; Wells et al., 1997; Winn and Wells, 1997). However, the subtle effects stemming from environmentally relevant concentrations of PAHs have yet not being characterized. The known differences in murine and human renal development make accurate comparisons of $\mathrm{BaP}$ exposure challenging. Our exposure regimen accounts for the normalization of species differences in renal developmental duration and approximates likely maternal exposures that are 
Figure 4. AHR expression correlates with deficits in nephrogenesis.

Kidneys were resected from 7-day old C57 mice exposed to either $0.1,0.5 \mathrm{mg} / \mathrm{kg}$ $\mathrm{BaP}$ or MCT oil in utero. Tissue was fixed in $4 \%$ paraformaldehyde and processed for modified Hematoxylin and Eosin staining, to visualize differentiated structures. Serial sections were processed for immunohistochemical analysis of AHR protein and counter stained with hematoxylin. AHR signal was calculated by creating a thresholded value for AHR signal accounting for nonspecific signal from negative control serial sections processed without primary antibody and primary antibody neutralized with blocking peptide. Sum density was normalized to area and statistical significance was calculated by ANOVA and LSD post hoc tests $p<0.05$. Panels $A$ and $B$ show that exposure to BaP decreases $A H R$ protein levels in a dose-dependent manner. Scale represents $200 \mu \mathrm{m}$. 
Figure 4

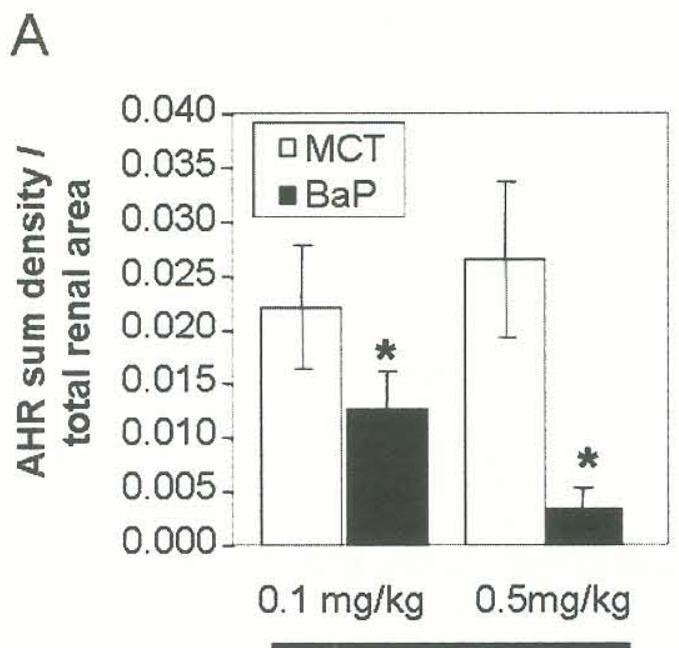

C57

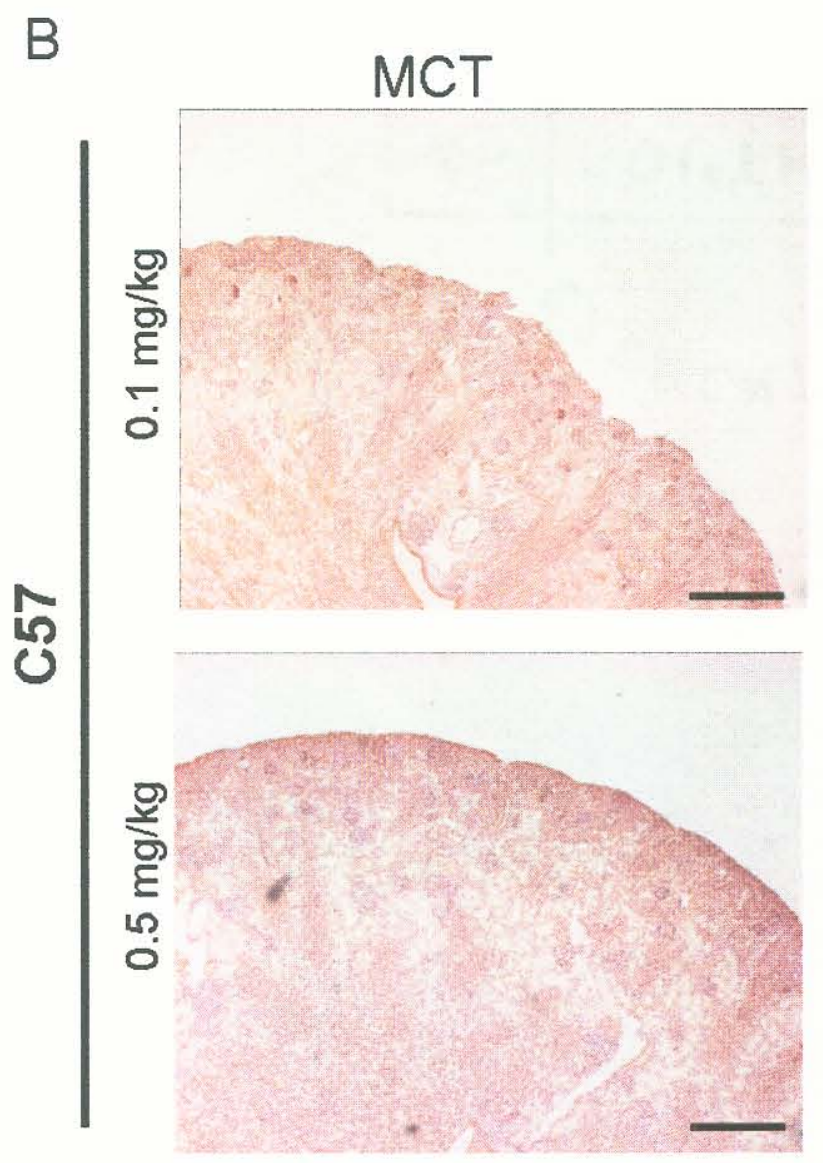

$\mathrm{BaP}$

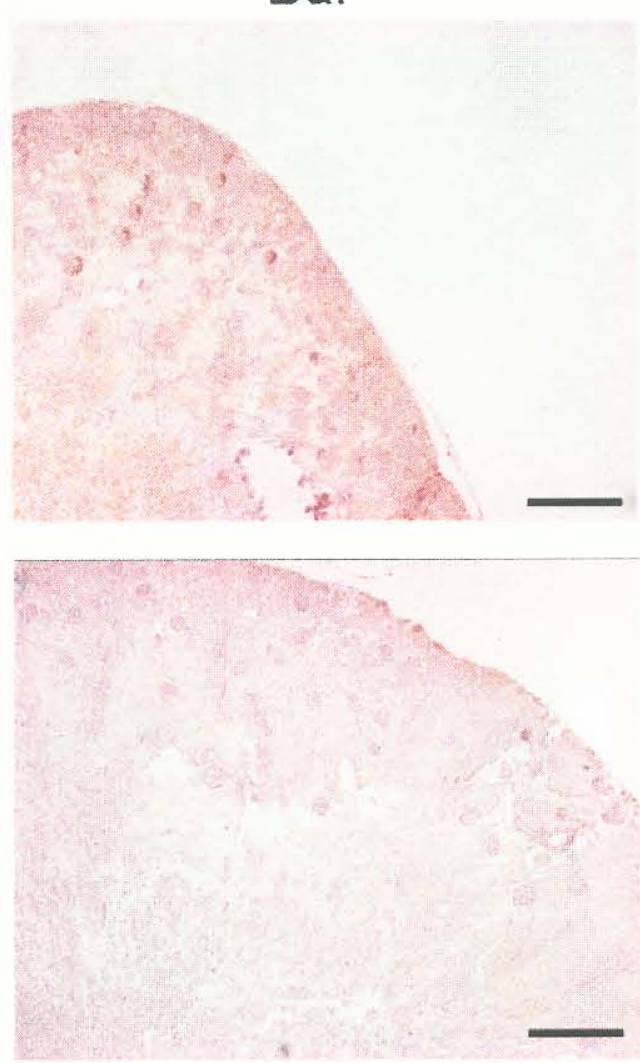


functionally relevant to human polymorphisms affecting PAH metabolism and AHR signaling (Sasaki et al., 2006; Wong et al., 2001a; Wong et al., 2001b).

Evidence is presented here that maternal exposure to $\mathrm{BaP}$ inhibits renal development in offspring via an AHR allele-specific mechanism characterized by decreases in glomerular and podocyte numbers that compromise glomerular filtration. Thus, the bioavailabity of PAHs presents a ready source for agents that can modify important early determinants of renal development. Specifically, the data shown here indicate that BiaP exposures of 0.1 and $0.5 \mathrm{mg} / \mathrm{kg}$ inhibit murine nephrogenesis specifically in C57 but not D2N mice. These findings are in agreement with previous work demonstrating that repeated exposure to environmentally-relevant concentrations of $\mathrm{BaP}$ inhibit nephrogenesis of murine metanephric cultures as evidenced by decreases in the number of renal differentiated structures and molecular markers of cell differentiation (Falahatpisheh and Ramos, 2003; Nanez and Ramos, 2007). Classical studies used gross endpoints such as fertility, fetal resorptions, death and severe developmental gonadal and immune deficits (Harrison et al., 1994; Lummus and Henningsen, 1995; MacKenzie and Angevine, 1981a; Nicol et al., 1995; Rodriquez et al., 1999; Wells et al., 1997; Winn and Wells, 1997). While our exposure regimen did not result in gross embryonic deficiencies, as measured by resorption, death, or morphological organ abnormalities, subtle BaP-induced differences become manifest as decreased glomerular numbers and 
compromised renal function. These deficits are relevant to the development of adult onset renal disease (Sasaki et al., 2006; Zandi-Nejad et al., 2006).

Our model in which maternal BaP insult results in decreases in podocyte number that impair glomerular filtration is strikingly similar to clinical manifestations seen in conditions such as focal segmental glomerulosclerosis (FSGS) or diabetic nephropathy (Hara et al., 2001; Hayden et al., 2005; Kim et al., 2001; Wolf et al., 2005). Podocytes are terminally differentiated cells that line the glomerular basement membrane (GBM) acting as a filter-mediated sizeselectivity and must structurally encompass the entire glomerular surface with foot processes to maintain proper filtration (Wiggins, 2007). Podocytopenia results in denudation of the GBM that compromise glomerular function initiating a cycle of injury and continued podocyte loss leading to progressive renal failure. In fact, glomerulopathies are the most common causes of end-stage renal disease worldwide (Hricik et al., 1998). The impact of in utero BaP exposure on other glomerular cell populations such as mesangial and endothelial cells remains to be determined. Of interest, are findings from in vivo studies in female Sprague-Dawley rats exposed by gavage to weekly $10 \mathrm{mg} / \mathrm{kg}$ BaP resulted in progressive elevations in total urinary protein, protein/creatinine ratios and microalbuminuria (Nanez et al., 2005). The nephropathic response involved early reductions in mesangial cell numbers coupled to podocyte injury as evidenced by effacement of foot processes. 
Of importance to our work is the evidence linking PAH exposures to renal injury. In humans, strong associations have been reported between occupational aromatic hydrocarbon exposures and glomerular diseases (Hotz, 1994; Robertson, 1998), smoking and albuminuria in nondiabetic patients (PintoSietsma et al., 2000), and a higher risk of renal dysfunction and renal cancer (Bertazzi et al., 1989). Taken together with our findings, PAHs may affect renal development and may predispose offspring to further injury resulting from repeated exposure in adulthood.

AHR signaling is implicated in BaP-induced toxicity as D2N mice expressing the nonresponsive $\mathrm{AHR}^{\mathrm{d}}$ allele are protected from nephrogenic deficits. Since the AHR ${ }^{d}$ allele is functionally null and BaP-induced deficits correlate to AHR protein expression, it is likely that loss of the AHR protein and constitutive signaling regulates nephrogenesis irrespective of nuclear translocation, association with ARNT, or AHRRE transcriptional activation. AHR transcriptional activity is regulated by association of complexes including AHRARNT, NF-kappa B, AP-1, and glucocorticoid, all of which participate in numerous signaling pathways potentially relevant to nephrogenesis. Our work has identified AHR as a regulator of the Wilms' tumor transcription factor, a master regulator that initiates genetic events regulating early nephrogenesis. Loss of the AHR through ligand activation, genetic mutation, or siRNA-mediated degradation results in dysregulation of WT1 mRNA isoforms and loss of renal cell differentiation markers (Falahatpisheh and Ramos, 2003; Falahatpisheh and 
Ramos, 2007; Nanez and Ramos, 2007). Recent studies have proposed that AHR acts as an E3 ligase associating with proteasomal complexes consisting of DDB1, ARNT, TBL3 and CUL4B promoting degradation of sex steroid hormone receptors (Ohtake et al., 2007). These findings suggest a scenario in which AHR regulates proteasomal degradation of factors necessary for renal development, a hypothesis that remains to be tested.

Expression of AHR is conserved across vertebrate and invertebrate species, many of which are not normally exposed to classical aromatic ligands (Hahn, 2002; Hahn et al., 2006). Our findings showing a correlation between the loss of AHR expression and compromised development (Nanez and Ramos, 2007), are in agreement with deficits seen in AHR-/- mice. However, these findings are not consistent with decreased levels of AHR protein seen in D2N mice-treated with $\mathrm{BaP}$ in utero. Thus, the nature of molecular signaling events regulated by AHR remain to be established. Irrespective of this observation, the collective evidence supports the hypothesis that AHR acts as an important nuclear transcription factor during embryogenesis and throughout maturity in multiple organisms. AHR-/- mice exhibit deficits in vascular structures stemming from a failure of the ductus venosus to close and result in reduced liver weight, microvesicular fatty metamorphosis, prolonged extramedullary hematopoeisis, and portal hypercellularity (Fernandez-Salguero et al., 1995; Lahvis et al., 2000; Lahvis et al., 2005; Schmidt et al., 1996). Of direct relevance to our studies are experiments in which AHR-/- mice were found to have cardiac hypertrophy and 
elevated mean arterial pressure, conditions known to arise from compromised renal function (Lund et al., 2003; Lund et al., 2006). We have previously shown that AHR- $/$ - mice exhibit delayed nephrogenesis resulting in compromised renal development, as evidenced by nephropenia and decreases in renal vasculature (Falahatpisheh and Ramos, 2003; McMillan and Bradfield, 2007b). Small interfering RNA (siRNA) mediated degradation of AHR results in decreases in renal cell differentiation markers (Falahatpisheh and Ramos, 2007). The mechanisms by which AHR signaling regulates renal cell differentiation and nephrogenesis remains poorly understood and, therefore, additional studies are warranted. 


\section{CHAPTER 3}

\section{AHR SIGNALING AND NEPHROGENESIS: A MUTANT AHR ALLELE PROTECTS THE DEVELOPING KIDNE:Y FROM HYDROCARBON-INDUCED DEFICITS IN RENAL CELLULAR DIFFERENTIATION}

\section{Summary}

The molecular mechanisms governing ontogenesis are regulated by somatic and environmental factors in utero that activate or repress the expression of numerous genetic elements. The aryl hydrocarbon receptor (AHR) is an important ligand-activated nuclear transcription factor involved in the regulation of cellular differentiation. Environmental hydrocarbons acting as AHR ligands deregulate receptor signaling to induce deleterious developmental deficits in several organ systems, including the kidney. Using a metanephric kidney organ culture system we have shown that benzo(a)pyrene (BaP), an AHR ligand, inhibits nephrogenesis in embryonic day 11.5 (Ell.5) C57BL/6J (C57) mouse embryos and alters -KTS/+KTS WT1 mRNA ratios after a consecutive four-day exposure. Because WT1 (Wilms' tumor suppressor-1) functions as a master switch in the regulation of renal cell differentiation, here we tested the 
hypothesis that modulation of AHR signaling by BaP mediates changes in WT1 splice variant ratios and expression of downstream effectors of WT1 leading to deficits in nephrogenesis. The data show that altered WT1 mRNA ratios in C57BL/6J mice expressing the $\mathrm{AHR}^{\mathrm{b}}$ allele correlated with a reduction in the mRNAs of multiple WT1 transcriptional targets (Synd1, Pax2, Egfr and Rara), as well as downstream markers of renal cell differentiation (Sfrp1, Igf1r, Igf2r, Wnt4, Lhx1, and E-cad). Metanephroi from B6.D2N-AHRd/J mice expressing an AHR variant allele of reduced ligand-binding affinity were spared deficits in renal cell differentiation following $\mathrm{BaP}$ treatment. We conclude that $\mathrm{BaP}$ disrupts nephrogenesis in vivo by altering the expression of downstream WT1 targets secondary to changes in the abundance of WT1 mRNA splice variants. These findings establish a link between WT1 and AHR signaling in the regulation of renal cell differentiation during the course of metanephric development.

\section{Introduction}

Embryonic development requires orchestration of temporally precise genetic events that culminate in the formation of a complete organism. The molecular mechanisms responsible for ontogenesis are regulated by environmental and somatic factors in utero that activate or repress the expression of numerous genetic elements. Recent studies have identified the 
aryl hydrocarbon receptor (AHR) as an important nuclear transcription factor during embryogenesis and throughout maturity in multiple organisms. AHR is involved in the regulation of development of vascular structures and liver (Fernandez-Salguero et al., 1995; Lahvis et al., 2005; Schmidt et al., 1996), with AHR-/- exhibiting cardiomegaly and hypertension, as well as renal and ocular deficits (Falahatpisheh and Ramos, 2003; Fernandez-Salguero et al., 1997; Lund et al., 2003; Lund et al., 2006; McMillan and Bradfield, 2007b). The discovery of endogenous substrates of AHR has further strengthened the hypothesis that AHR functions as a critical developmental regulator (McMillan and Bradfield, 2007a). Potent environmental chemicals acting as AHR ligands disrupt normal signaling to exert deleterious effects during embryonic development (Mackenzie and Angevine, 1981b; Ng et al., 2006b; Ng et al., 2006c; Shum et al., 1979; Yu et al., 2006). While the mechanism of AHR-mediated regulation of xenobiotic metabolism is well understood, the details of AHR function during development remain unclear.

AHR is a transcription factor activated by hydrophobic aromatic hydrocarbons. Upon ligand binding, AHR dissociates from two molecules of the chaperone heat shock protein 90 (HSP90), and binds the X-associated protein 2 (XAP2) and p32. The resulting complex exposes a nuclear localization sequence that facilitates nuclear translocation. This complex in turn binds to the aryl hydrocarbon receptor nuclear translocator (ARNT or HIF1 $\beta$ ) resulting in DNA binding to sequences containing 5'-GCGGGGGCG-3' and (TCC) $)_{n}$ repeats 
(Denison and Nagy, 2003). Translocation of the AHR-ligand complex to the nucleus triggers its proteolytic degradation regardless of ARNT binding (Roberts and Whitelaw, 1999). AHR is also regulated by negative feedback inhibition in which the nuclear AHR complex activates transcription of the AHR repressor protein (AHRR) responsible for binding the xenobiotic response element (XRE) and preventing transcriptional activation by AHR (Mimura et al., 1999).

A role for AHR in renal development was established in experiments showing that AHR-null mice exhibit deficits in renal condensation, appearance of differentiated structures and cellular proliferation (Falahatpisheh and Ramos, 2007). Renal development proceeds in three morphologically distinguishable stages pronephros, mesonephros, and metanephros (Pohl et al., 2000). The kidney derives from the nephric ridge in the intermediate mesoderm. The pronephric duct gives rise to the pronephros which elongates to form the Wolffian duct. The Wolffian duct develops into the ureteric bud setting the stage for the key event in nephrogenesis where mesonephric mesenchyme is stimulated to undergo condensation and mesenchymal-to-epithelial cell transition. A host of genes including, WT1, glial cell derived neuronal factor (GDNF) and bone morphogenic protein-7, are responsible for regulating this process (Kuure et al., 2000). While removing any one of a host of proteins can result in impaired nephrogenesis, the molecular mechanisms involved have yet to be fully characterized. 
Of interest is the central role of Wilms' tumor suppressor gene (WT1) in nephrogenesis. WT1 encodes a $\mathrm{Cis}_{2}-\mathrm{His}_{2}$ zinc fingered protein that functions via transcriptional regulation of target genes, including insulin-like growth factor II, syndecan-1, epidermal growth factor receptor and retinoic acid receptor- $\alpha$ (Hosono et al., 1999). In addition to its role in tumorigenesis, WT1 is considered a "master switch" that regulates mesenchymal-to-epithelial differentiation during renal development (Avner, 1993). Visceral epithelial cells (podocytes) are the only cell type in adult kidney that retains WT1 expression. Homozygous WT1 -/mice do not develop normally, die in utero, and do not show differentiation of metanephric kidney from the existing pronephros (Kreidberg et al., 1993b). WT1 activity can be regulated in cis by different ratios of its own splice variants, or in trans by proteins such as bone marrow zinc finger 2 (BMZF2) (Lee et al., 2002). Perhaps the most studied regulatory mechanism involves the formation of WT1 \pm KTS splice variants. KTS splice variants originate from the insertion of a lysinethreonine-serine between the third and fourth zinc finger. The resulting change modifies the WT1 DNA binding specificity leading to activation and/or repression of target genes (Menke et al., 1998b). In humans, reduced WT1 +KTS mRNA isoforms result in severe kidney and gonad developmental deficits, collectively known as Frasier syndrome (Barbaux et al., 1997). Changes in exon 5 splice variants have also been associated with deficits in renal differentiation (Iben and Royer-Pokora, 1999). Addition of 17 amino acids in exon 5 creates an mRNA isoform that regulates transactivation (Wang et al., 1995). N-terminal residues 1- 
182 encode a dimerization region implicated in the regulatory mechanism exerted by dominant negative mutants (Englert et al., 1995; Reddy et al., 1995).

The regulation of renal cell differentiation is controlled by synchronized expression of multiple genes involved in control of the developmental programming. Sfrp1 is a mesenchymal marker expressed in the metanephric medullary and cortical stroma that acts as an inhibitor of the mesenchymal-toepithelial cell transition (Yoshino et al., 2001a). Igf2r is chiefly expressed during the early stages of nephrogenesis whereas Igf $1 \mathrm{r}$ is expressed ubiquitously throughout renal maturation (Dong Van Huyen et al., 2003). Loss of function of either receptor compromises renal differentiation (Feld and Hirschberg, 1996). Wnt4 is expressed in the condensed mesenchyme specifically around the ureter tip and in pretubular aggregates (for review see (Vainio et al., 1999). Wnt4 deficiency inhibits epithelial tubule development resulting in dysfunctional nephrons (Stark et al., 1994). Lim-1 is a homeobox transcription factor essential for early kidney morphogenesis. Lim-1 is chiefly expressed in the ureteric bud, induced mesenchymal aggregates, and differentiated structures (comma and Sshaped bodies) (Barnes et al., 1994; Karavanov et al., 1998). Murine models with Lim-1 deficiency are born headless and without kidneys despite the presence of other organs (Shawlot and Behringer, 1995b). E-cad is a general marker of the mesenchymal-to-epithelial cellular transition and is expressed in the ureteric bud epithelium, distal tubule progenitor cells and the majority of differentiated tubular epithelium (Cho et al., 1998). 
The present study was conducted to evaluate the role of AHR signaling in murine nephrogenesis. This work builds upon previous studies establishing a novel link between AHR and post-transcriptional regulation of WT1 (Falahatpisheh and Ramos, 2003). Evidence is presented that the low affinity $\mathrm{AHR}^{\mathrm{d}}$ allele abrogates BaP-induced molecular changes in the developing kidney, including changes in WT1 splicing and expression of WT1 downstream effectors involved in regulation of renal cell differentiation.

\section{Methods}

\section{Metanephric Culture}

Day 11.5 mouse embryos were surgically dissected from C57BL/6J $A H R^{b}$ wild type and B6.D2N-AHR ${ }^{d / J}$ mice. Embryonic kidneys were dissected and cultured on $0.45 \mathrm{~mm}$ polyethylene trephalate cyclopore cell culture inserts (Fisher) for 1-4 days. The use of transparent membranes allowed for direct visualization of tissue by phase contrast microscopy. Kidney explants were maintained at the liquid-gas interface in a solution consisting of a 1:1 mixture of Dulbecco's modified Eagle's medium (DMEM) and F12 supplemented with $10 \%$ fetal bovine serum (FBS) and a $5 \mathrm{X}$ concentration of MITO serum extender (Becton Dickinson, Bedford MA). Explants were allowed to equilibrate for 1 day before initiation of experiments. Seven or more embryonic kidney explants from 
4 dams were placed on individual inserts and exposed daily to $3 \mu \mathrm{M} \mathrm{BaP}$ or an equivalent volume of DMSO with or without $20 \mathrm{nM}$ a-napthoflavone for 1,3 , and 4 days. The $3 \mu \mathrm{M} \mathrm{BaP}$ dose is an environmentally relevant exposure that provides optimum activation of the AHR receptor (Bowes and Ramos, 1994). At the end of each time point, the explants were fixed in situ for evaluation.

\section{Cell culture}

mK4 cells were cultured in DMEN (Cellgro) supplemented with $10 \%$ fetal bovine serum (Atlanta Biologicals) and 1\% antibiotic-antimycotic (Gibco). Cells were seeded at staggered time intervals at an initial density of $40 \mathrm{cell} s / \mathrm{mm}^{2}$ to adjust final densities in culture. After equilibration for 24 hours, cells were exposed $3 \mu \mathrm{M}$ BaP or DMSO for 9,15 , or 51 hours.

\section{Histology and Morphometric Analysis;}

Metanephroi were fixed in $4 \%$ paraformaldehyde at $4^{\circ} \mathrm{C}$ for 16 hours, immobilized in Histogel (Richard Allen Scientific) and embedded in paraffin. 4 $\mu \mathrm{m}$ serial sections were cut and processed for modified hematoxylin and eosin staining allowing for visualization of differentiated structures. Images of at least 5 metanephroi per treatment group were captured at $5 \mathrm{X}$ with an Axiovert 200 inverted microscope (Zeiss) and stored as .zvi files. Glomeruli, S-shaped, and comma-shaped bodies were quantified using manual functions on Axiovision Rel 4.1 (Zeiss). 


\section{Immunohistochemistry}

AHR was detected using a rabbit polyclonal antibody (B-1) (Biomol) applied overnight at $4^{\circ} \mathrm{C}$ in a solution containing $0.3 \%$ Triton $\mathrm{X}$ and $10 \%$ goat serum (Vector Laboratories). Primary antibody was detected using a goat antirabbit biotinylated secondary antibody (Molecular Probes), amplified with ABCelite $\AA$, developed with $\mathrm{DAB}$, and counterstained with Mayer's Hematoxylin (Vector Laboratories).

\section{Quantitative PCR}

Total RNA was extracted using TRIzol ${ }^{\circledR}$ and cDNA was synthesized using SuperScript II (Invitrogen, Carlsbad, CA) as per manufacturer's instructions. Quantitative PCR using primers specific for WT1 isoforms was performed to detect differences in the ratio of WT1 splice variants ( \pm KTS) in response to AHR ligand treatment (Falahatpisheh and Rarnos, 2003; Falahatpisheh and Ramos, 2007). All other primers were designed using Beacon Designer 5.1 to create amplicons from 150-300 base pairs with an average melting temperature of $55^{\circ} \mathrm{C}$

si RNA

Ambion Silencer Pre-designed siR2NA for AHR (162529 and 88685), WT1 (18990 and 100360), and a scrambled negative control (\#4635) were transfected using Fugene 6 (Roche, Indianapolis, IN). Briefly, $150 \mathrm{nM}$ of each specific siRNA was incubated with transfection reagent daily and applied to cells seeded at a 
density of 60 cells $/ \mathrm{mm}^{2}$ for three days. Seventy-two hours after treatment, cells were harvested in situ for further analysis.

\section{Statistical Analysis}

Statistical significance was determined using Students t-test, ANOVA, Wilcoxon Rank Sums, LSD, and Tukey post hoc tests at the $p<0.05$ level.

\section{Results}

Deficits in metanephric differentiation by BaP are linked to AHR signaling

The activation of $\mathrm{AHR}$ signaling by $\mathrm{BaP}$ culminates in transcriptional activation/repression of target genes, and proteasomal degradation of AHR protein (for reviews see (Denison and Nagy, 2003; Fujii-Kuriyama and Mimura, 2005; Pocar et al., 2005). To examine the role of AHR signaling in BaP-induced deficits in nephrogenesis, metanephric explants from C57-AHR ${ }^{\mathrm{b}}$ (C57) and D2N$\mathrm{AHR}^{\mathrm{d}}$ (D2N) mice were challenged with $3 \mu \mathrm{M} \mathrm{BaP}$ for 1,3 , or 4 days. C57 and $\mathrm{D} 2 \mathrm{~N}$ mice are isogenic strains that differ in AHR ligand binding affinity due to a single nucleotide substitution that replaces valine for alanine at codon 375 in the ligand binding domain resulting in a 5-fold reduction in ligand binding affinity (Poland et al., 1994). Quantitative reverse transcriptase polymerase chain reaction (QRT-PCR) analysis of C57 metanephroi revealed time-dependent 
induction of Cyp1a1 mRNA, while D2N metanephroi expressing the variant AHR allele showed a markedly abrogated response (Table 1). A 4-day BaP exposure resulted in compromised metanephric development (Figure 1A), as evidenced by decreased number of glomeruli (Figure 1B), and predominance of lesser differentiated, comma and S-shaped bodies (Figure 1C). To determine if ligandbinding mediated deficits in renal cell differentiation, D2N mice expressing the $\mathrm{AHR}^{\mathrm{d}}$ allele, or $\alpha$-napthoflavone ( $\left.\alpha-N F\right)$, a competitive inhibitor of the AHR ligand binding site, were tested. Interference with AHR-ligand interactions prevented BaP-induced deficits in renal differentiation. a-NF alone modulated renal development outcomes (Figure 1B). Immunohistochemical analysis showed a decrease in AHR protein after 4 days of $\mathrm{BaP}$ exposure in $\mathrm{AHR}^{\mathrm{b}}$ mice (Figures $2 \mathrm{~A}$ \& B). In sharp contrast, the abundance of $A \mathrm{HR}^{\mathrm{d}}$ was not influenced by hydrocarbon treatment (Figure $2 \mathrm{~A}$ ). The $\mathrm{AHR}^{\mathrm{d}}$ phenotype, or co-treatment with $a-N F$, prevented the loss of AHR protein, suggesting that modulation of nephrogenesis by BaP directly correlates with depletion of AHR protein. This finding is consistent with the hypothesis that AHR is required for renal developmental signaling, and that disruption of nephrogenesis requires AHR ligand binding, activation of AHR signaling, and protein degradation.

Next, the expression of mesenchymal (Sfrp1) and epithelial (Igf1r, Wnt4, Lhx1, and E-cad) markers of differentiation was monitored by QRT-PCR to determine if morphological deficits correlated with modulation of these genes. Markers of renal epithelial cell differentiation (Igf1r, Wnt4, Lhx1, and E-cad) were 
Table 1. E 11.5 metanephroi were resected from C57 and D2N-AHR ${ }^{d}$ mice and cultured on $0.45 \mu \mathrm{m}$ inserts for 4 days in the presence of $3 \mu \mathrm{M} \mathrm{BaP}$ or DMSO. At 1,3 , and 4 days individual metanephroi were homogenized and processed for RNA isolation and cDNA synthesis. Quantitative PCR was performed with values representing $\triangle \Delta C T$ normalized fold change relative to DMSO control (dashed line). Variance represents standard deviation between no less than 6 metanephroi per group. Statistical significance was calculated using a Wilcoxon rank sum. Cyp1a1 mRNA was inudced.in a dose and time-dependent manner in both $\mathrm{C} 57$ and D2N mice but to a greater extent in $\mathrm{C} 57$. 
Table 1. Cyp1a1 mRNA Expression

Normalized fold change

Exposure (days) relative to DMSO Control

$\begin{array}{ccc} & 1 & 154.34 \pm 20.8 \\ \text { C57 } & 3 & 455.09 \pm 30.62 \\ & 4 & 730.00 \pm 24.00 \\ & 1 & 1.82 \pm 0.27 \\ \text { DBA } & 3 & 12.44 \pm 2.03 \\ & 4 & 39.49 \pm 1.20\end{array}$


Figure 1. BaP inhibits nephrogenesis via an AHR allele-specific mechanism. E 11.5 (days post conception) metanephroi were resected from C57 and D2N-AHR ${ }^{d}$ mice and cultured on $0.45 \mu \mathrm{m}$ inserts for 4 days in the presence of $3 \mu \mathrm{M}$ BaP or DMSO $\pm \alpha-N F$. At 4 days, metanephroi were fixed in $4 \%$ paraformaldehyde and processed for modified hematoxylin and eosin staining to visualize differentiated structures. Panel A, BaP-exposed metanephroi show less morphologically distinct differentiated structures compared to $\mathrm{C} 57$ control. Expression of the AHR ${ }^{d}$ allele in D2N mice abrogates BaP-induced deficits. Co-treatment with the competitive inhibitor a-NF also inhibited BaP effects. B, quantitation of number of glomeruli normalized to area from serial sections of no less than 6 metanephroi per group. C, quantitiation of comma and S-shaped bodies normalized to area. Statistical significance was calculated by ANOVA and LSD post hoc tests at a $p<0.05$ level. 
Figure 1
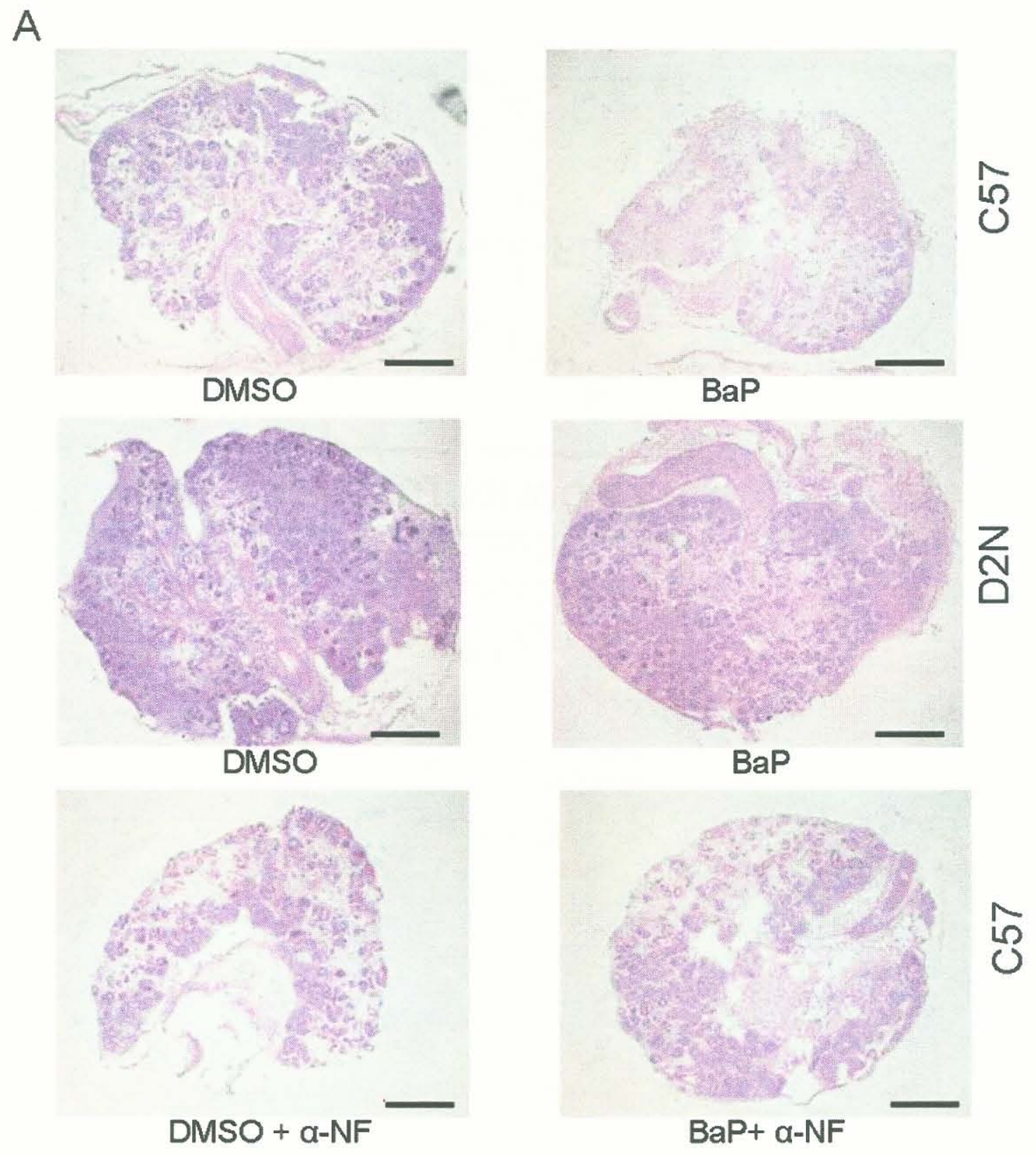
Figure 1

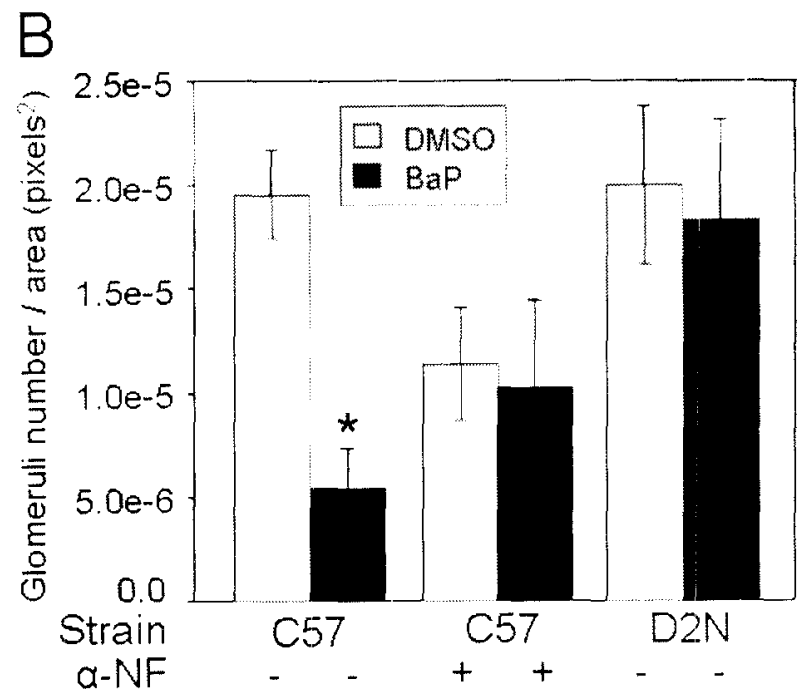

C

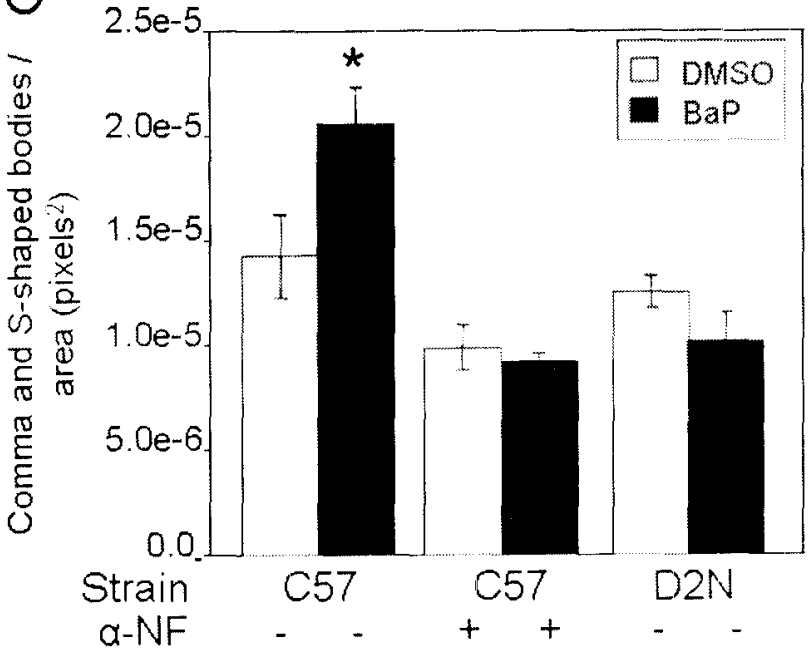


Figure 2. AHR expression correlates with nephrogenesis. E 11.5 metanephroi were resected from $\mathrm{C} 57$ and D2N-AHR ${ }^{\mathrm{d}}$ mice and cultured on 0.45 $\mu \mathrm{m}$ inserts for 4 days in the presence of $3 \mu \mathrm{M} \mathrm{BaP}$ or DMSO $\pm \alpha-N F$. At 4 days, metanephroi were fixed in $4 \%$ parafomaldeyde and processed for modified hematoxylin and eosin staining, to visualize differentiated structures. Serial sections were processed for immunohistochemical analysis of AHR protein and counterstained with hematoxylin. AHR signal was calculated by creating a thresholded value for AHR signal accounting for nonspecific signal from negative control serial sections processed without primary antibody and primary antibody neutralized with blocking peptide. Sum density was normalized to area and statistical significance was calculated by ANOVA and LSD post hoc tests $p<$ 0.05. Panels $A$ and $B$ show that exposure to $B a P$ decreases $A H R$ protein levels. Protein expression was similar in C57 and D2N metanephroi, however D2N or aNF co-treated metanephroi were not sensitive to BaP-induced deficits in AHR protein expression. 
Figure 2
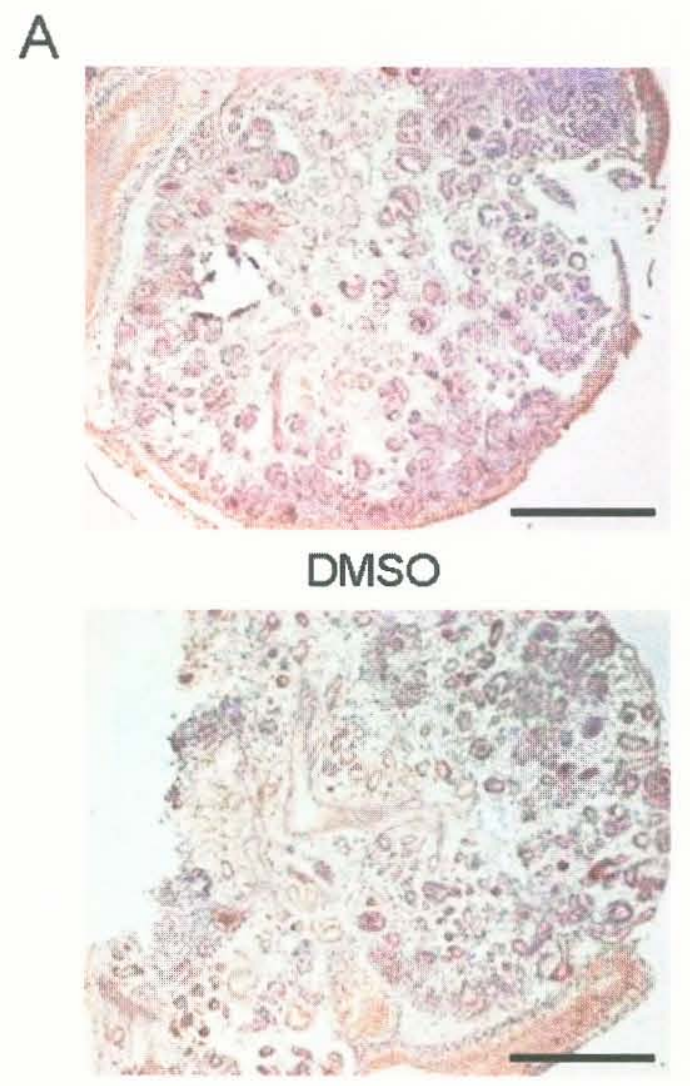

DMSO
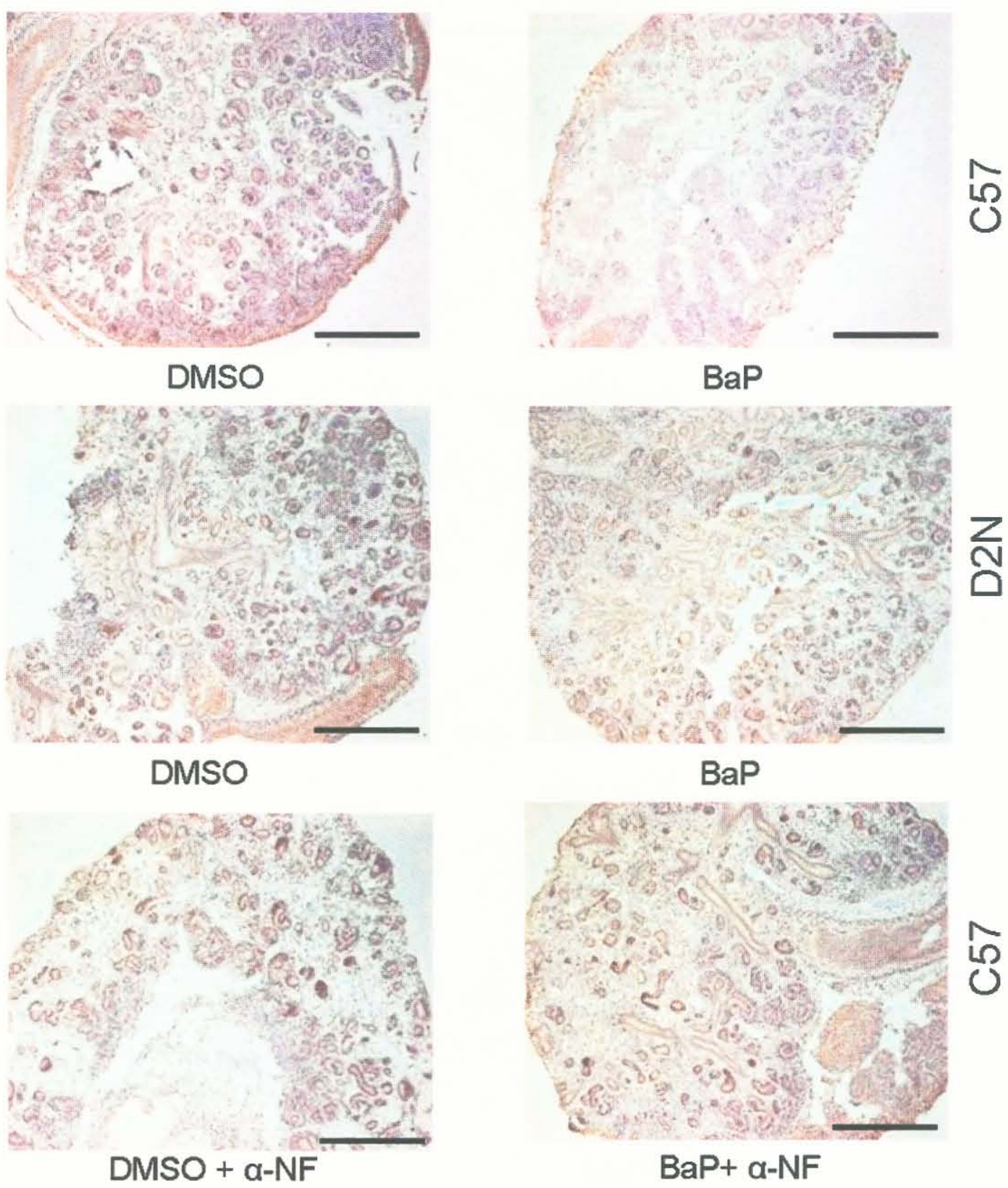

$\mathrm{BaP}$

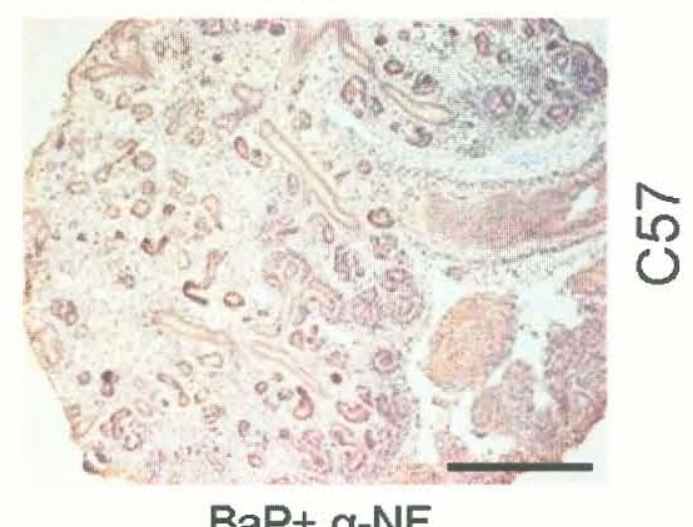

$\mathrm{BaP}+\alpha-\mathrm{NF}$ 
Figure 2

B

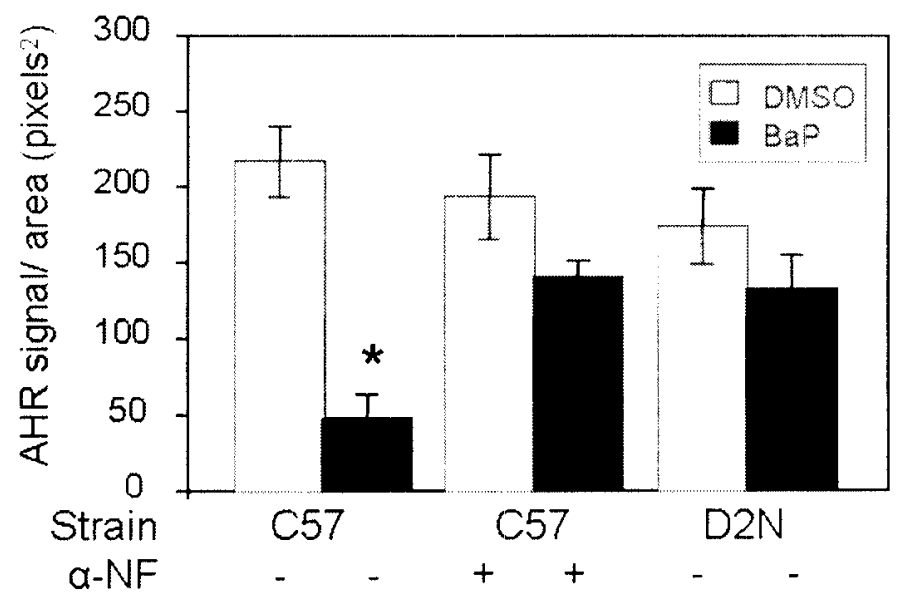


induced to varying degrees relative to vehicle control after 1 day of BaP exposure (Figure 3A). In C57 metanephroi, Igf1r, Igf2r, Wnt4, Lhx1, and E-cad mRNA levels were decreased after 3 and 4 days of BaP treatment (Figure $3 \mathrm{~A}$ ). No timedependent changes in the expression of the mesenchymal marker Sfrp1 were seen in C57 mice where downregulation was seen at all time-points compared to vehicle (Figure 3A). Metanephroi expressing the $A H R^{d}$ allele showed variable degrees of induction in Sfrp1, Igf1r, Wnt4 and E-cad throughout the exposure and were spared reductions of all markers examined, except Lhx1 (Figure 3B) These results suggest that nephrogenic deficits induced by BaP require activation, and integrity, of AHR signaling.

The AHR allele mediates disruption of WT1 mRNA splice variants by BaP

Previous work established that exposure of C57 mouse metanephric kidney cultures to BaP for 4 days modulates the expression of -KTS/+KTS WT1 mRNA variants (Falahatpisheh and Ramos, 2003). A 4-day BaP exposure of C57 metanephroi resulted in 3- and 7-fold induction of +KTS and -KTS variants, respectively (Figures $4 A$ and $B$ ). Expression of the +17 aa or -17 aa was not altered by BaP treatment (Figures $4 \mathrm{C}$ and $\mathrm{D}$ ). Consistent with the known WT1 transcriptional repressive activity, significant reductions in the relative expression of several WT1 targets including: Synd1, Pax2, Egfr, and Rara, were observed (Figure 4E). D2N-AHR ${ }^{d}$ metanephroi exposed to $3 \mu \mathrm{M} \mathrm{BaP}$ did not exhibit changes in any of the WT1 mRNA splice variants (Figures 5A-D) or WT1 targets (Figure 5E), except for Rara where the pattern of gene regulation was reversed 
Figure 3. BaP exposure downregulates markers of renal cell differentiation.

E 11.5 metanephroi were resected from C.57 and D2N-AHR ${ }^{d}$ mice and cultured on $0.45 \mu \mathrm{m}$ inserts for 4 days in the presence of $3 \mu \mathrm{M} \mathrm{BaP}$ or DMSO. At 1, 3, and 4 days individual metanephroi were homogenized and processed for RNA isolation and cDNA synthesis. Quantitative PCR was performed with values representing $\triangle \triangle \mathrm{CT}$ normalized fold change relative to DMSO control (dashed line). Variance represents standard deviation between no less than 6 metanephroi per group. Statistical significance was calculated using a Wilcoxon rank sum. Panels $A$ and $B$ show, that at 4 days $B a P$ exposure, significant decreases in differentiation markers were seen in C57, but not in D2.N metanephroi. 
Figure 3
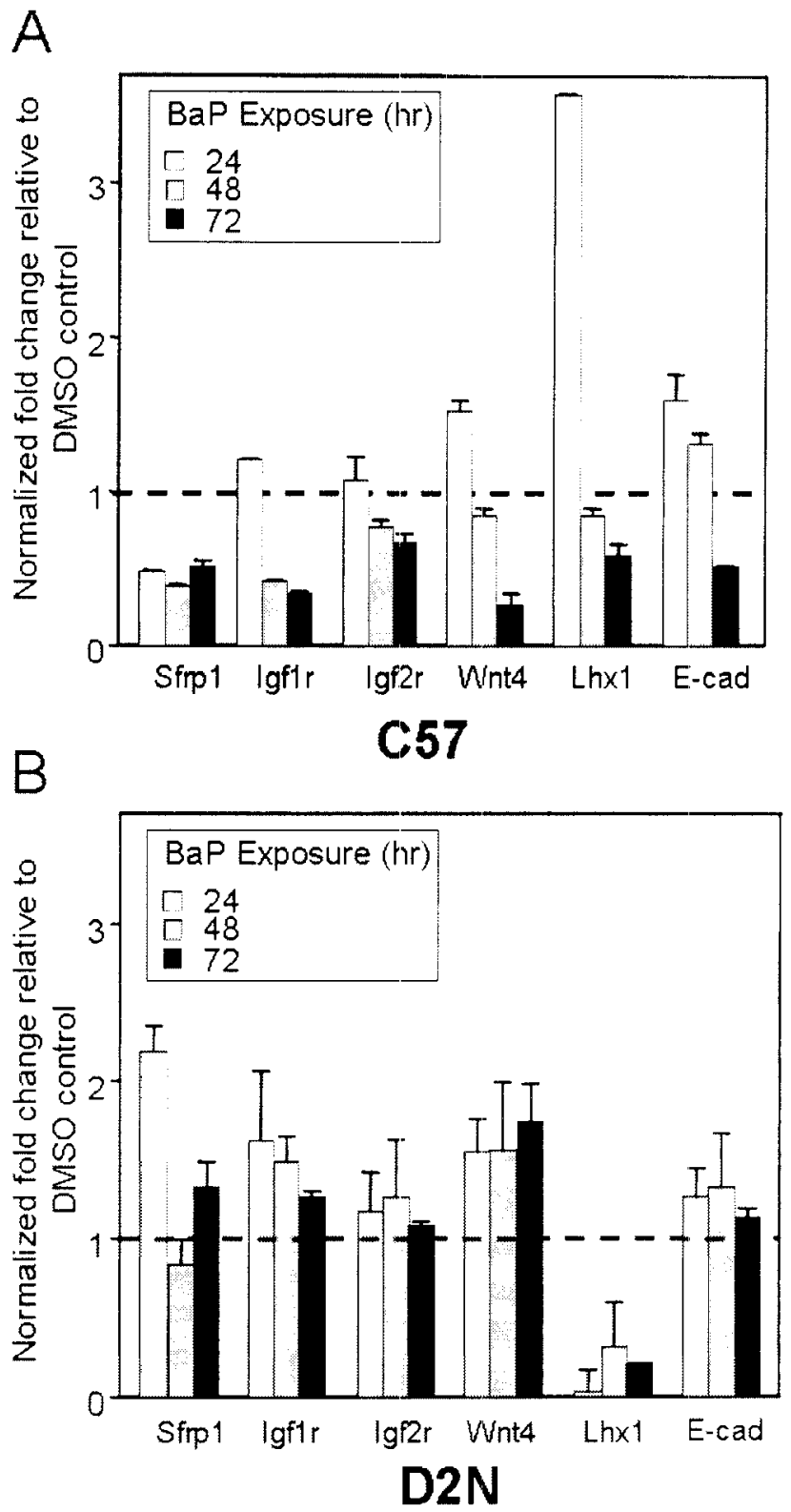
Figure 4. BaP-induced WT1 dysregulation correlates with loss of known WT1 targets. E 11.5 metanephroi were resected from C57 mice and cultured on $0.45 \mu \mathrm{m}$ inserts for 4 days in the presence of $3 \mu \mathrm{M}$ BaP or DMSO. On days 1,3 and 4 , individual metanephroi were homogenized and processed for RNA isolation and cDNA synthesis. Quantitative PCR was performed with values representing $\triangle \Delta C T$-normalized fold change relative to DMSO control (dashed line). Variance represents standard deviation between no less than 6 metanephroi per group. Statistical significance was calculated using a Wilcoxon rank sum. Panels A-D, primers specific for each splice were used to compare the abundance of splice variants from BaP treated and DMSO control samples. No changes were seen in total WT1 mRNA expression. Panels A and B, Significant increases were seen in -KTS compared to +KTS after 4 days BaP exposure. Panels $C$ and $D$, no significant changes were seen in +17 aa or -17 aa isoforms. Panel E shows that downregulation of known WT1 targets correlated with changes in WT1 isoform abundance. 
Figure 4

A

B
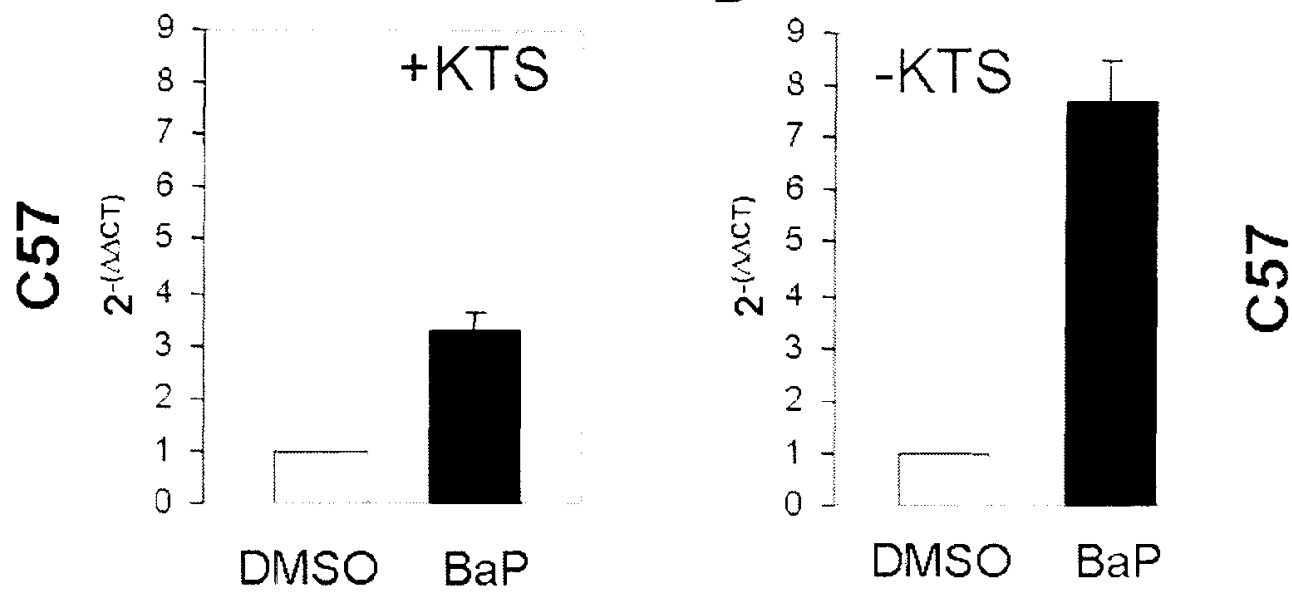

C

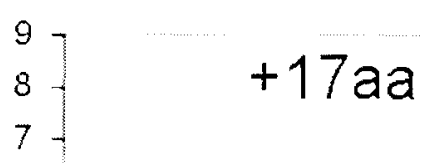

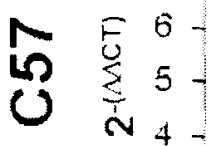

N 4 .

3.

2 -

1 -

0 -

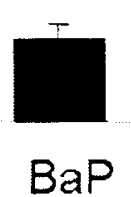

DMSO BaP

D
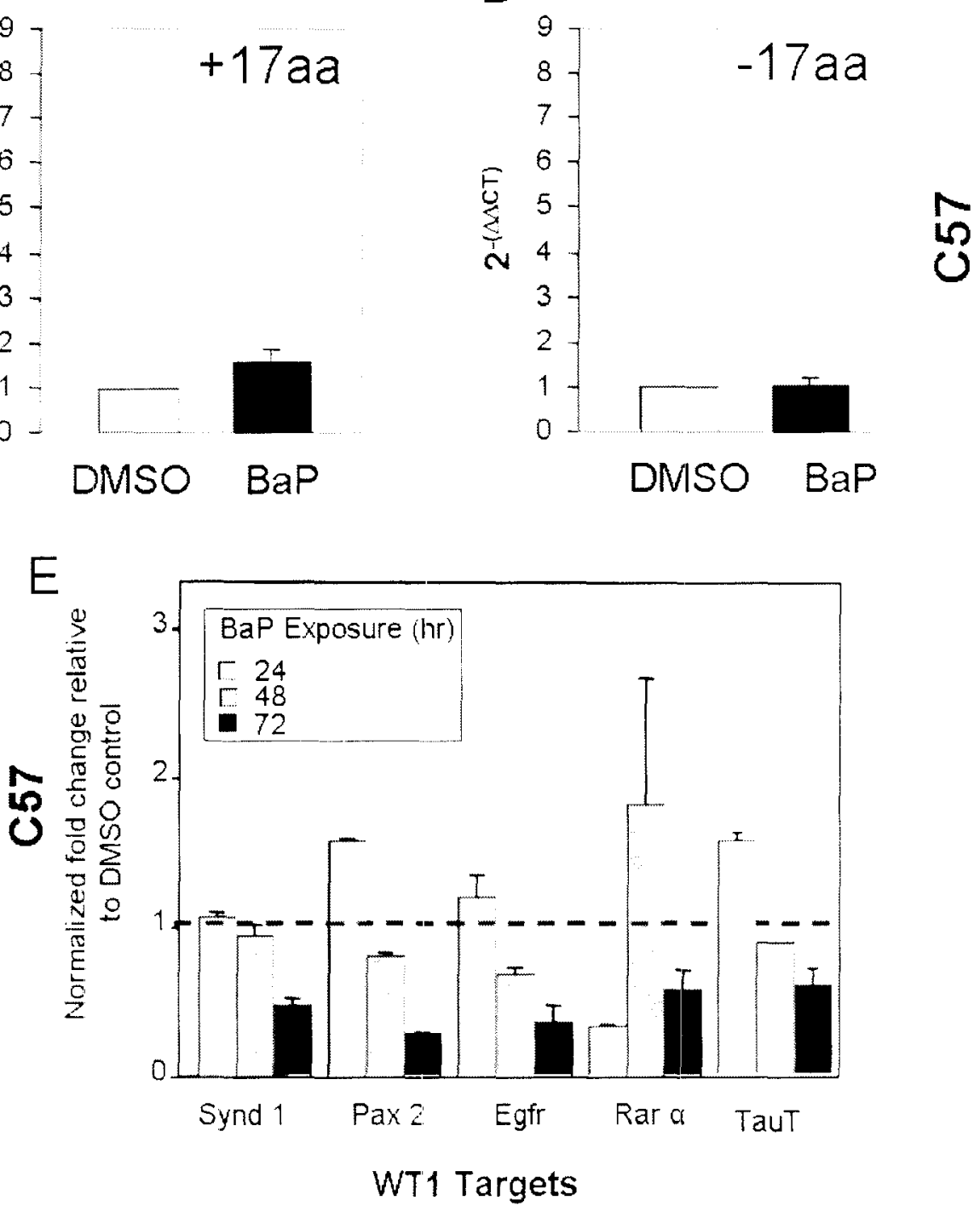
Figure 5. BaP-induced WT1 dysregulation is AHR allele-specific. E 11.5 metanephroi were resected from D2N-AHRd mice and cultured on $0.45 \mu \mathrm{m}$ inserts for 4 days in the presence of $3 \mu \mathrm{M} \mathrm{BaP}$ or DMSO. On days 1,3 , and 4 , individual metanephroi were homogenized and processed for RNA isolation and cDNA synthesis. Quantitative PCR was performed with values representing $\Delta \Delta C T$-normalized fold change relative to DMSO control (dashed line). Variance represents standard deviation between no less than 6 metanephroi per group. Statistical significance was calculated using a Wilcoxon rank sum. Panels A-D, primers specific for each splice were used to compare the abundance of splice variants from BaP treated and DMSO control samples. No changes were seen in total WT1 mRNA expression. Panels A - D, expression of the AHR ${ }^{d}$ allele abrogates BaP-induced modulation of WT1 mRNA isoforms in D2N mice. Panel E, downregulation of known WT1 targets correlates with changes in WT1 isoform abundance. 
Figure 5
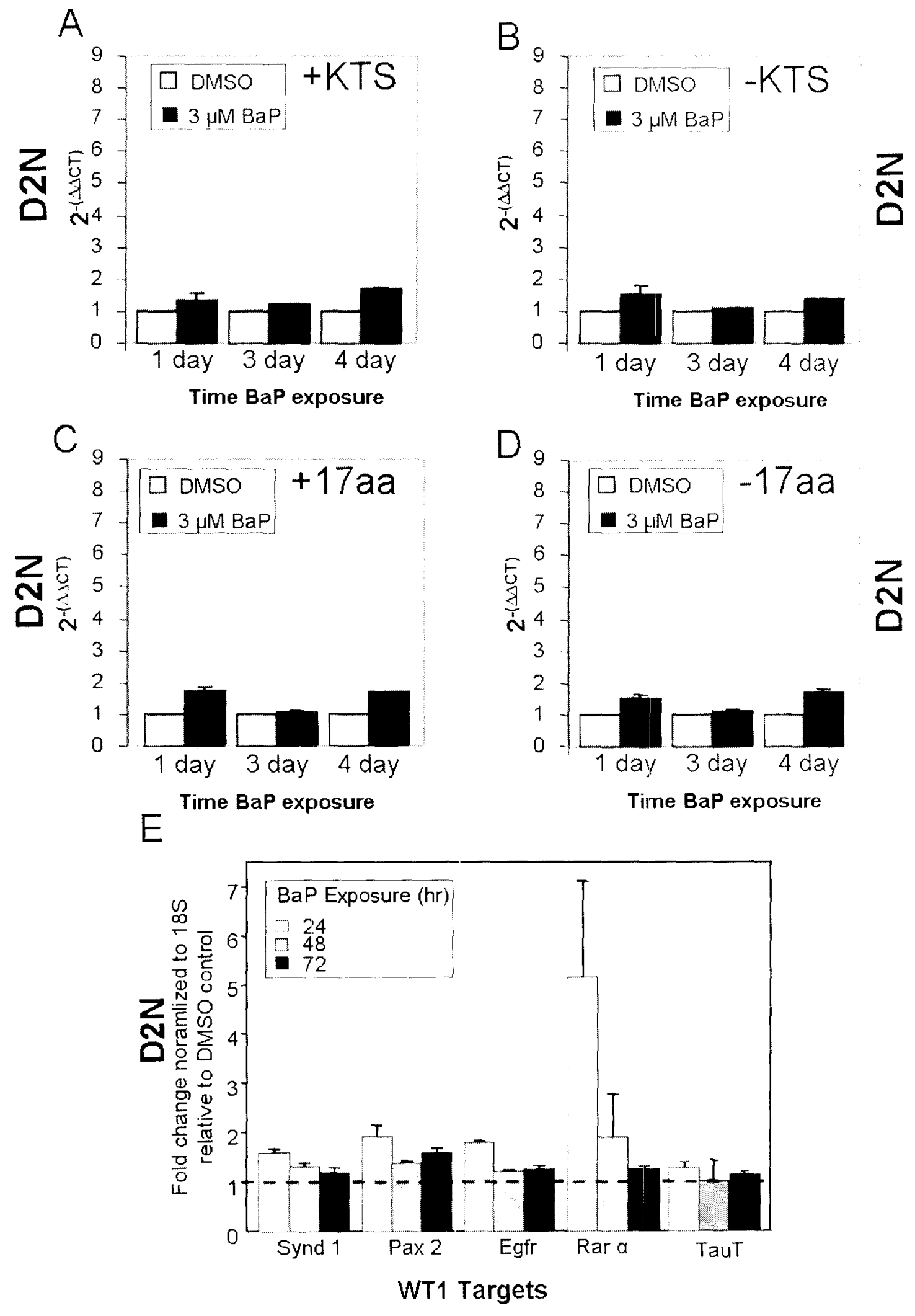
compared to the C57 strain. C57 metanephroi exhibited decreases in taurine transporter mRNA and these changes were absent in D2N metanephroi exposed to $\mathrm{BaP}$ (compare Figures $4 \mathrm{E}$ and $5 \mathrm{E}$ ).

mK4 metanephric cells are sensitive to modulation of WT1 mRNA isoforms by BaP and other AHR ligands

To develop a model amenable to molecular manipulation, mouse mK4 cells derived from metanephric tissue were exposed to $3 \mu \mathrm{M} \mathrm{BaP}$ as a function of time (Valerius et al., 2002). mK4 cells exhibited time-dependent induction of Cyp1a1, with maximal effects seen $51 \mathrm{hr}$ after hydrocarbon exposure (Figure 6A). Sfrp1, Igf1r, Igf2r, Wnt4, and E-cad were downregulated by BaP as a function of time (Figure 6B), with the most prominent effects seen for Sfrp1, Igf1r, Wnt4 and E-cad. Interestingly, Sfrp1 and Lhx1, markers of the mesenchymal phenotype contain AHR response elements in their promoter regions (data not shown), and showed a biphasic response. Changes in markers of renal cell differentiation correlated with changes in +KTS and - KTS where 2- and 5-fold inductions in mRNA expression, respectively, were observed (Figures 7A and B). The expression of the +17 aa isoform exhibited a biphasic, response, with induction seen at 9 and 15 hours and a return to baseline at 51 hours (Figures 7C and D). As in the metanephric model, AHR protein loss (Figure 7E) and dysregulation of WT1 splice variants correlated with reductions in WT1 target mRNAs, as evidenced by reductions in Pax2, Egfr, and Rara at 51 hours of BaP exposure (Figures 7F). 
Figure 6. $\mathrm{mK} 4$ cells are sensitive to BaP induced changes in differentiation markers. mK4 cells were seeded and exposed at various times to $3 \mu \mathrm{M} \mathrm{BaP}$ or DMSO to allow for harvest at equal density. At 9,15 , and 51 hours cells were processed for RNA isolation and cDNA synthesis. Quantitative PCR was performed with values respresenting $\Delta \Delta C T$-normalized fold change relative to DMSO control (dashed line). Variance represents standard deviation amongst at least three biological replicates. Statistical significance was calculated using a Wilcoxon rank sum. Panel A shows that $\mathrm{mK} 4$ cells display classical CYP1A1 profile induction with a maximal response at 51 hours. Panel B shows that after 51 hours of $\mathrm{BaP}$ exposure $\mathrm{mK} 4$ cells downregulated markers of differentiation. 
Figure 6

$A$

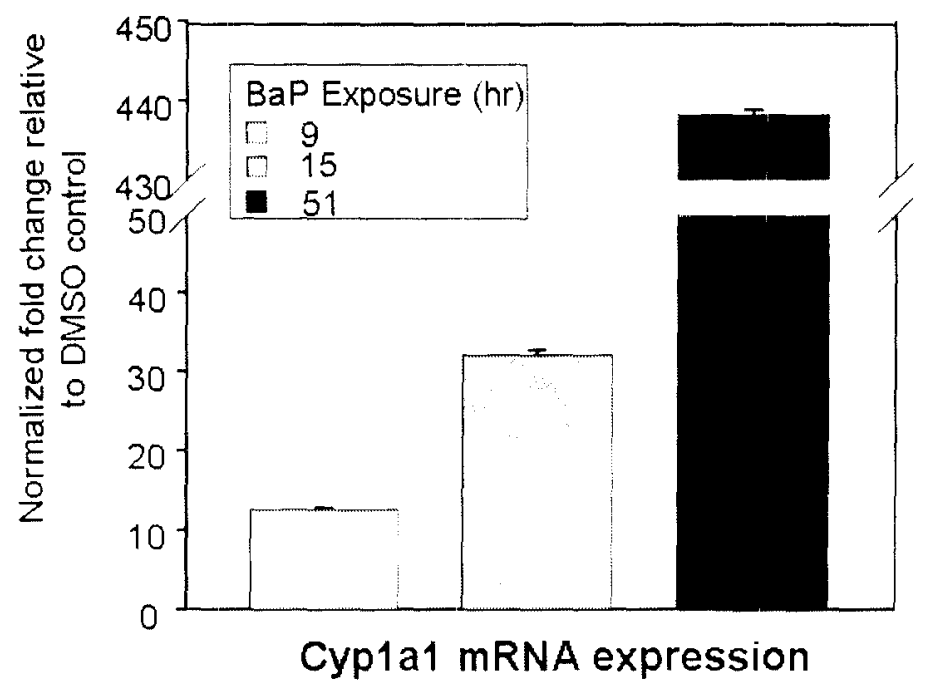

B

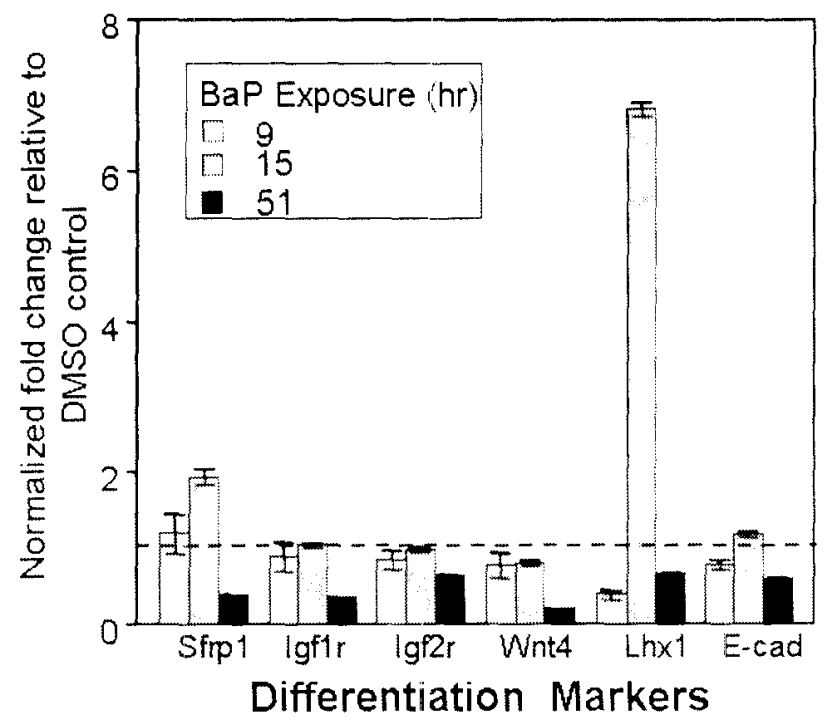


Figure 7. $\mathrm{mK} 4$ cells are sensitive to BaP induced modulation of WT1

status. mK4 cells were seeded and exposed at various times to $3 \mu \mathrm{M} \mathrm{BaP}$ or DMSO to allow for harvest at equal density. At 9,15 , and 51 hours cells were processed for RNA isolation and cDNA synthesis. Quantitative PCR was performed with values representing $\Delta \Delta \mathrm{CT}$-normalized fold change relative to DMSO control (dashed line). Variance represents standard deviation amongst at least three biological replicates. Statistical significance was calculated using a Wilcoxon rank sum. Panels $A$ and $B, 51$ hours of $B a P$ exposure induces - KTS. Panels $C$ and $D,+17$ aa is increased at 9 and 15 hours. Panel $E$, Western blot analysis of WT1 targets shows loss of protein for known WT1 markers. Panel F, Inductions in -KTS WT1 mRNA isoform correlate with decreases in WT1 targets. 
Figure 7

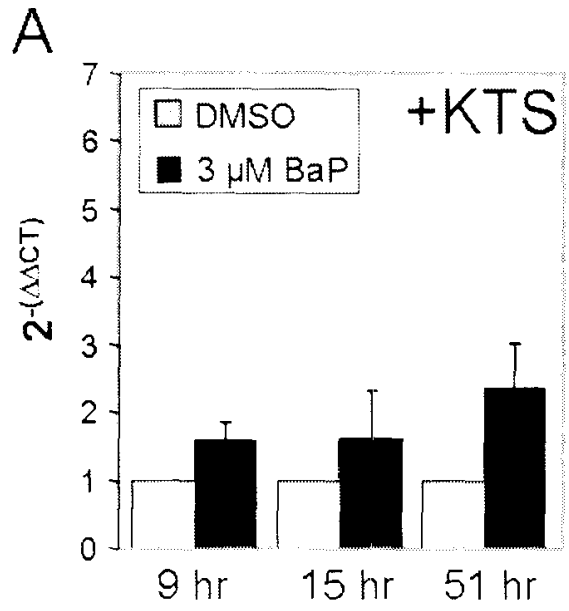

Time BaP exposure

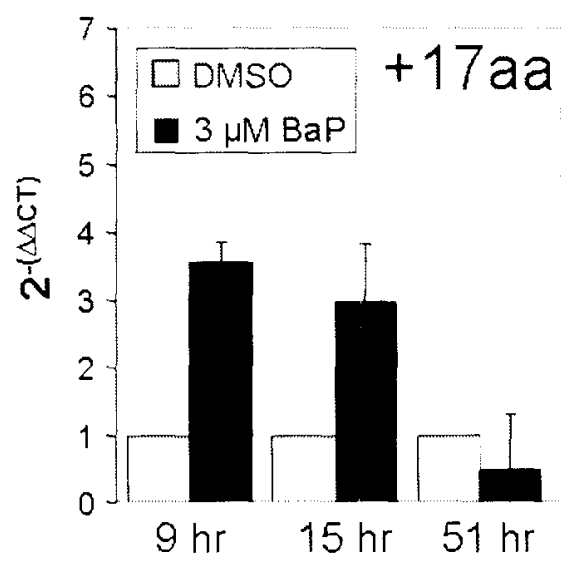

Time BaP exposure
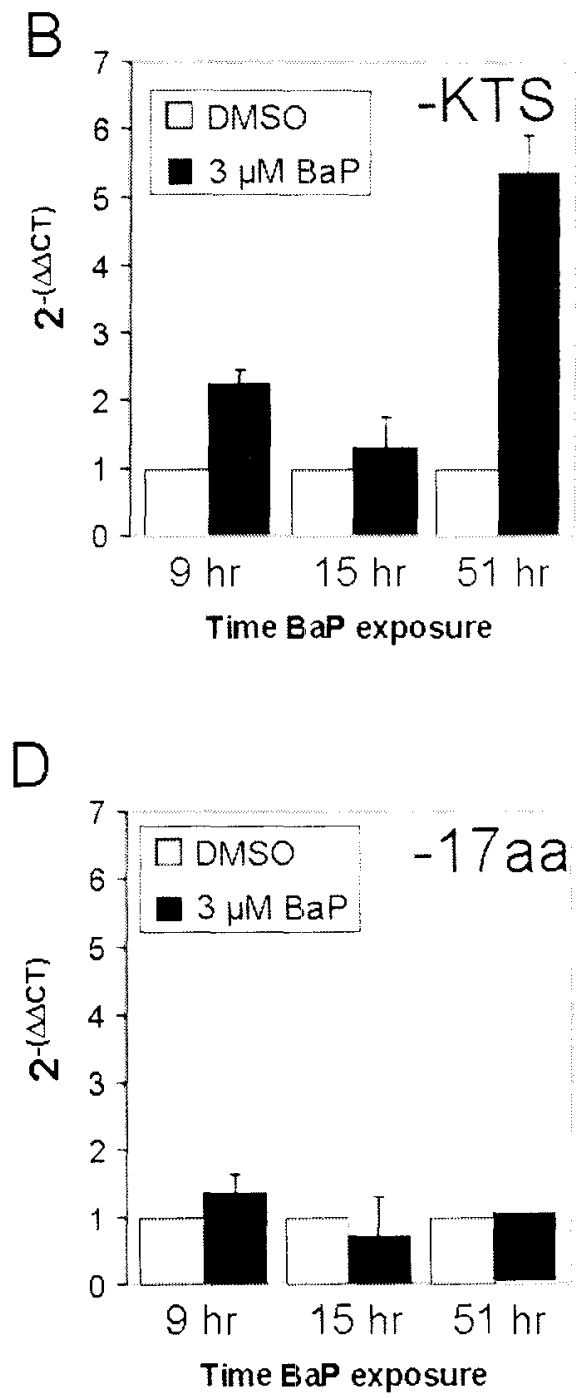
Figure 7

$E$

AHR

a-Tub

$$
\begin{array}{cccc}
\text { DMSO } & 9 & 15 & 51 \\
3 \mu \mathrm{m} & \text { BaP (hours) }
\end{array}
$$

F

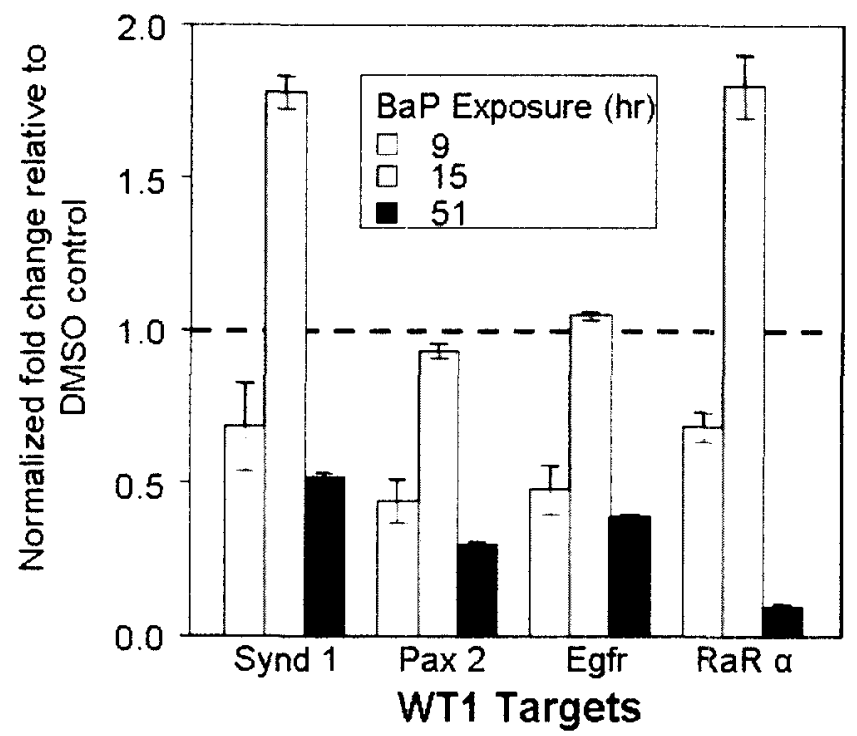


To determine if nephrogenic deficits were seen with other AHR ligands, mK4 cells were challenged with varying concentrations of TCDD ranging from 0.1 $\mathrm{pM}$ to $1 \mathrm{nM}$ for 48 hours. Integrity of AHR signaling was confirmed by induction of Cyp1a1 mRNA (Figure 8A). Both 100 pM and 1 nM TCDD decreased AHR protein (Figures 8B and C). Exposure to $100 \mathrm{pM}$ TCDD reduced expression of differentiation markers Sfrp1, Igf1r, Igf2r, and Wnt4 (Figure 8D). The loss of differentiation markers correlated with altered expression of WT1 splice variants (Figure 8E). WT1 target genes, Synd1, Pax2, Egfr, and Rara were also downregulated by TCDD exposure (Figure 8F).

\section{Discussion}

Evidence continues to mount implicating AHR in proliferation, development, adhesion and migration, and proteasomal degradation (for reviews see (Barouki et al., 2007; Ohtake et al., 2007)). In this regard, previous studies in this laboratory have shown that repeated exposure of cultured metanephros to $3 \mu \mathrm{M} \mathrm{BaP}$ results in significant impairment of metanephric development (Falahatpisheh and Ramos, 2003). Such deficits required AHR activation and appears to be linked to the protein degradation machinery of target cells in the developing kidney. While the E3 ligase activity of AHR has yet to be fully 
Figure 8. Alternative AHR ligands moderate differentiation markers and WT1 status. mK4 cells were seeded and exposed to TCDD or vehicle for 51 hours to allow for harvest at equal density. Cells were processed for RNA isolation, cDNA synthesis and protein analysis. Quantitative PCR was performed with values representing $\Delta \triangle \mathrm{CT}$ normalized fold change relative to DMSO control (dashed line). Variance represents standard deviation amongst at least three biological replicates. Statistical significance was calculated using a Wilcoxon rank sum. Panel A, dose-dependent Cyp1a1 mRNA induction. Panels B and C, western blot analysis reveals a dose-dependent loss of AHR protein. Panel D, TCDD exposure results in loss of renal cell differentiation markers. Panel E, +17aa WT1 mRNA is induced. Panel F, WT1 dysregulation correlates to loss of WT1 targets. 
Figure 8

B

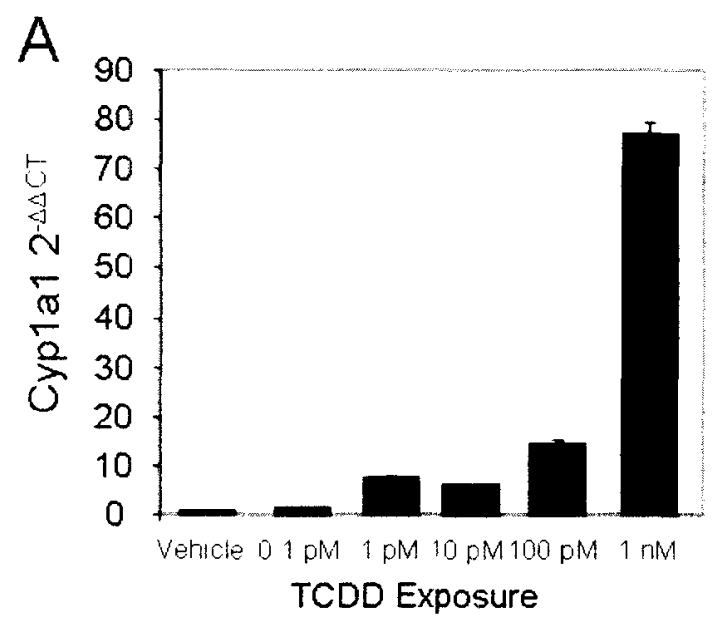

AHR

$a-T u b$

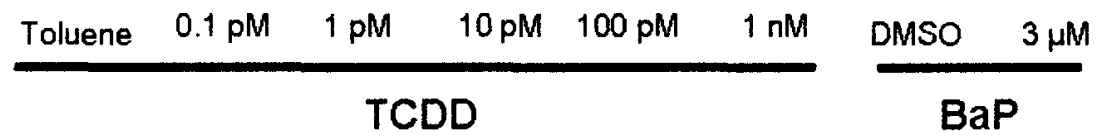

C

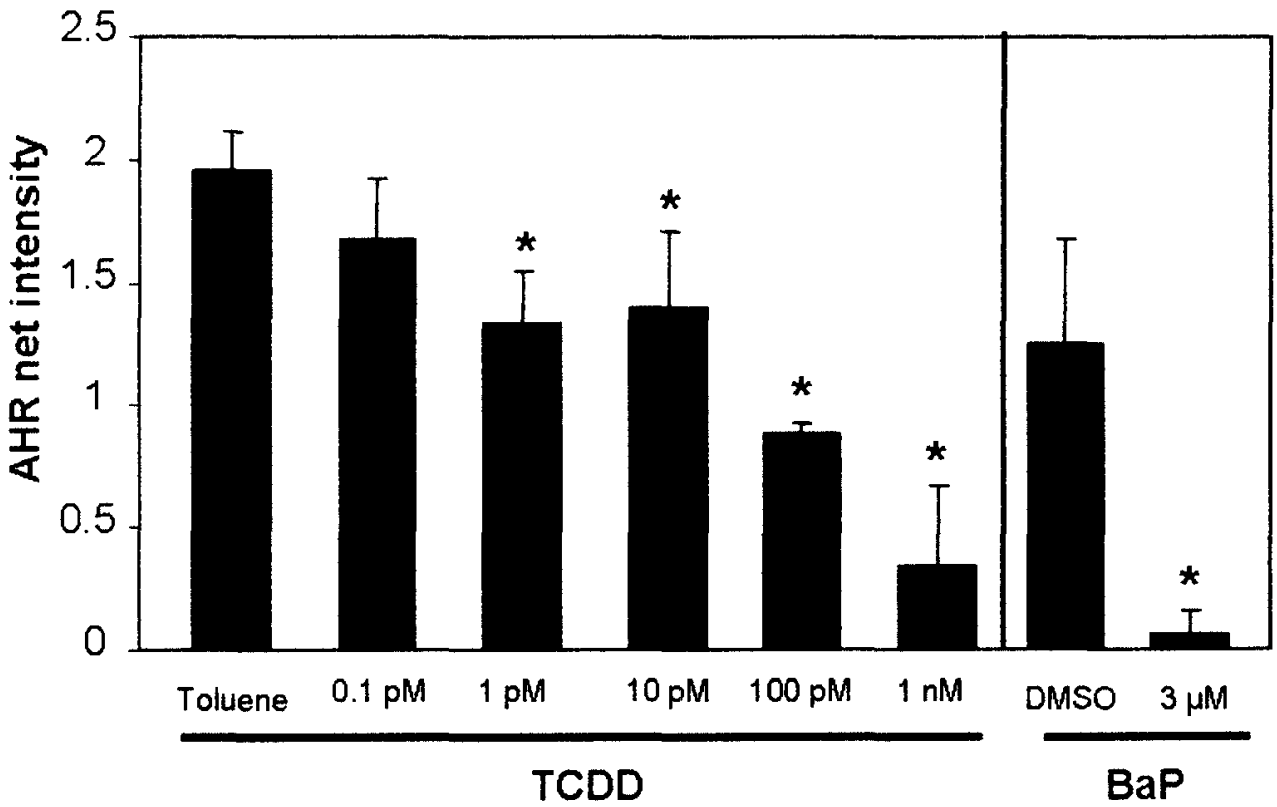


Figure 8

D

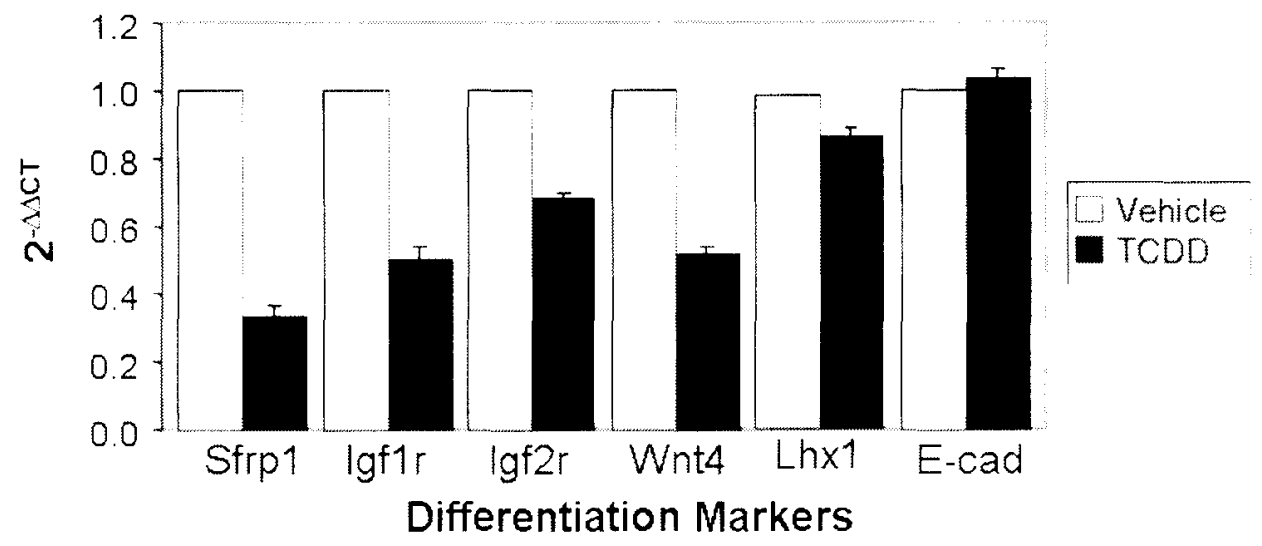

$E$

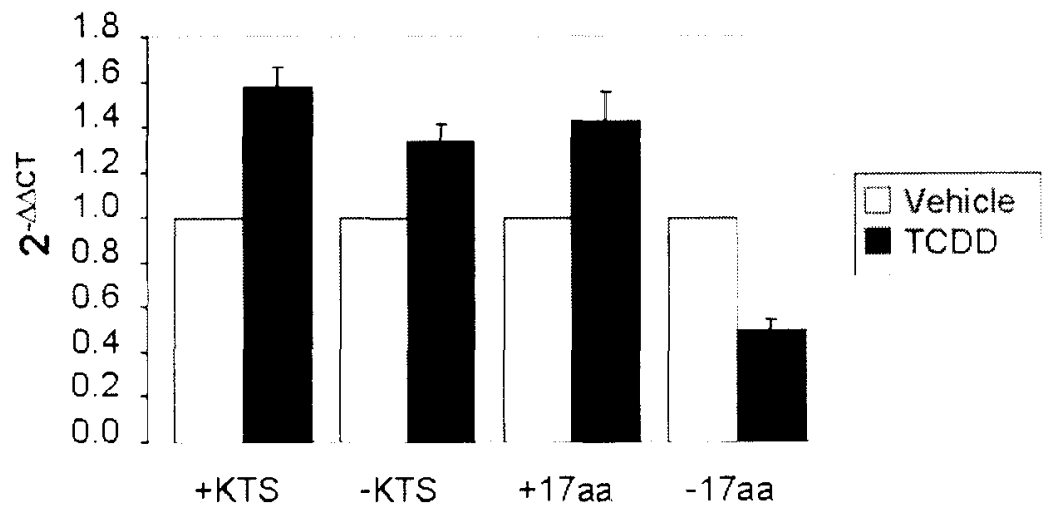

WT1 isoforms

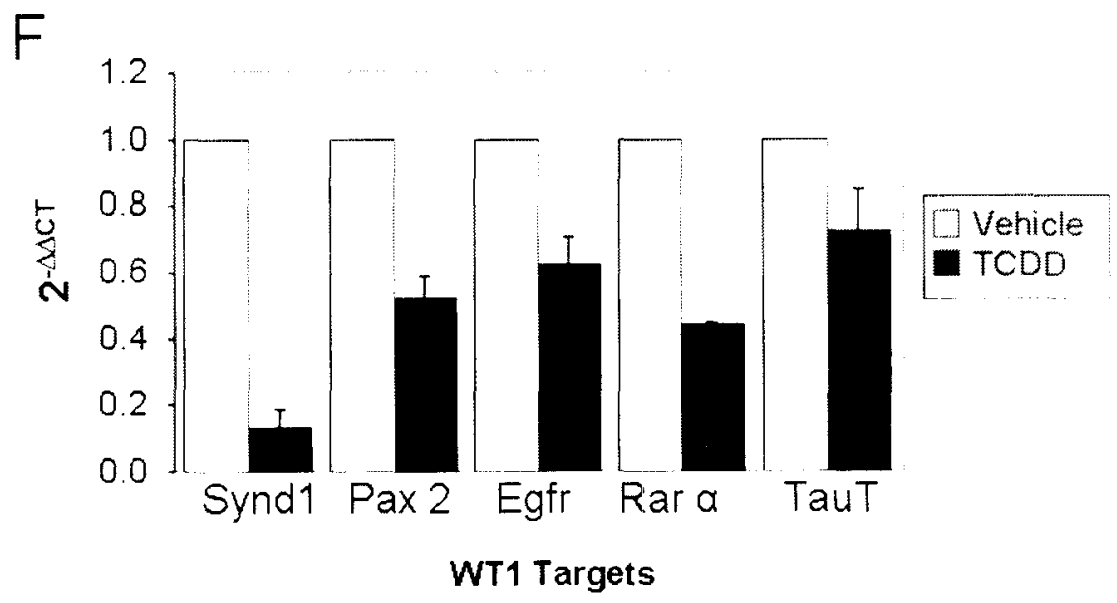


characterized (Ohtake et al., 2007), AHR ubiquitination and degradation can occur both in the cytoplasm (Song and Pollenz, 2002), or upon nuclear translocation (Roberts and Whitelaw, 1999), and ARNT/DNA binding (Song and Pollenz, 2003). Mutation of the ligand binding domain in the $A \mathrm{HR}^{\mathrm{d}}$ allele decreases ligand binding affinity by 5 -fold compared to $\mathrm{AHR}^{\mathrm{b}}$ and decreases nuclear translocation (Poland et al., 1994), transcriptional activation and degradation upon ligand binding. Similarly, AHR antagonists, such as $\alpha-N F$, do not induce the conformational changes necessary for translocation and/or degradation of $A H R^{b}$ (Henry and Gasiewicz, 2003). These relationships define a scenario in which constitutive AHR plays a regulatory role in renal development that is compromised upon ligand-mediated degradation of receptor protein. In fact, AHR-null mice exhibit delayed nephrogenesis and compromised renal development (Falahatpisheh and Ramos, 2003), and these changes can be reproduced by downregulation of AHR protein upon ligand binding. Of importance to the work presented here are studies in which AHR-null mice were found to be hypertensive, a condition that is directly related to deficits in renal function (Lund et al., 2003; Lund et al., 2006).

Our model detailing a constitutive function for AHR in regulation of nephrogenesis via WT1 is supported by the finding that prevention of AHR loss pharmacologically, or by the use of the genetically-resistant D2N strain, prevented renal developmental deficits in BaP-treated metanephros, and restored normal ratios of WT1 mRNA isoforms. Mice overexpressing the -KTS 
isoform have severely compromised renal development, evidenced by increased stromal tissue, decreases in tubular epithelium and glomerular tuft, and altered podocyte structure (Hammes et al., 2001). While the exact molecular consequences resulting from alterations of the four abundant and biologically relevant isoforms of WT1 remain to be fully defined, it is well-established that the -KTS mRNA encodes a protein that participates in transcriptional activity, and that protein encoding +KTS mRNA associates with splicing factors (Davies et al., 1998; Hastie, 2001). In both metanephros and mK4 cell cultures, BaP-induced increases in -KTS resulted in downregulation of differentiation markers, a finding consistent with the morphological deficits seen in -KTS transgenic mice. Restoring WT1 mRNA splice variant ratios via $\mathrm{AHR}^{\mathrm{d}}$ expression did not alter renal differentiation markers. Transgenic mice expressing solely +17 aa WT1 do not exhibit gross renal nephropathy (Natoli et al., 2002), but have deficits in other organ systems such as the olfactory system (Wagner et al., 2005).

The complexity of AHR functions in different transcriptional complexes such as the classical AHR- ARNT, NF-kappa B, AP-1, and glucocorticoid receptor provides possible mechanisms for regulation of WT1 splicing. Of these, the glucocorticoid receptor has been shown to associate with PGC-1, a known regulator of co-transcriptional splicing (Knutti et al., 2001). Another possibility involves direct binding of AHR to the WT1 promoter which contains two consensus AHR responsive elements within a regulatory region known to regulate transcription-coupled splicing events (Cohen et al., 1997). Ongoing 
experiments are examining whether AHR binds to the WT1 promoter to alter the splicing machinery directly (Bentley, 2002), or indirectly by altering elongation efficiency (Eperon et al., 1988; Roberts et al., 1998). Because AHR itself associates with proteasomal complexes consisting of DDB1, ARNT, TBL3 and CUL4B, and has E3 ligase activity (Ohtake et al., 2007), AHR may also regulate proteasomal degradation of factors required for WT1 splicing.

The reduction of selected renal differentiation markers seen in organ and cell culture is consistent with the loss of AHR protein mediated by BaP, TCDD, or small interfering RNA (data not shown). All markers examined play central roles in nephrogenesis. Lhx1-null mice do not progress past mesonephric development (Shawlot and Behringer, 1995a). Wnt4 is implicated in early nephron development and differentiation (Stark et al., 1994). Inhibition of the Igf1 $\mathrm{r}$ impairs kidney growth, as evidenced by a reduction in nephrons and altered ureteric bud branching (Murphy and Barron, 1993; Wada et al., 1993). E-cad-null mice have decreased nephron number due to a failure of proper fusion of the metanephric mesenchyme to the ureteric bud (Mah et al., 2000). Sfrp1, most likely through Wnt interactions, plays an important role in the mesenchymal to epithelial cell transition manifested during tubule formation and bud branching (Yoshino et al., 2001b). While these markers have yet to be examined in AHRnull mice, the preservation of AHR expression and constitutive signaling via expression of $\mathrm{AHR}^{\mathrm{d}}$ or treatment with antagonist, restores their expression. These findings associate functional molecular consequences resulting from 
disruption of constitutive AHR functions with the regulation of renal cell differentiation.

The discovery of novel functions of AHR during nephrogenesis, coupled to molecular interactions of AHR with WT1, highlight a potential mechanism for compromised renal development following AHR signaling interference. The bioavailability of PAHs presents a ready source for agents that can modify important early determinants of renal cell differentiation. Of importance to our work are studies linking AHR genetic polymorphisms to tobacco smoke induceddecreases in birth weight (Sasaki et al., 2006). Low birth weight compromises renal development resulting in increases in microalbuminuria, proteinuria, decreased glomerular filtration rate, and the occurrence of end stage renal disease (for review see (Zandi-Nejad et al., 2006)). Other stressors, such as diet and environmental exposures, are associated with lower birth weights and deficits in renal development (Everson et al., 1988; Nelson et al., 1999; Roquer et al., 1995; Zandi-Nejad et al., 2006). Preliminary findings indicate that exposure of pregnant $\mathrm{C} 57$ dams to $\mathrm{BaP}$ to environmentally-relevant concentrations, inhibits renal development (Nanez, et al, unpublished).

The present studies identify a novel role for AHR in the regulation of murine nephrogenesis. Expression of the $A H R^{d}$ allele preserves the integrity of AHR signaling and abrogates hydrocarbon-induced dysregulation of WT1 mRNA isoforms thus preserving renal cell differentiation signals. Thus, endogenous and 
exogenous modulators of AHR signaling during critical periods of nephrogenesis may alter the genetic programming of renal development. 


\section{CHAPTER 4}

\section{LOSS OF THE ARYL HYDROCARBON RECEPTOR PROTEIN ALTERS WT1 MRNA ISOFORM EXPRESSION AND DOWNSTREAM EFFECTORS OF RENAL CELL DIFFERENTIATION}

\section{Summary}

A key event in nephrogenesis is the transdifferentiation of mesenchymal cells to epithelial cells, a tightly regulated process controlled by the Wilms' tumor transcription factor (WT1). WT1 activity can be regulated in cis by different ratios of its mRNA splice variants, or in trans by accessory proteins recruited to regulatory regions of the gene. Recently, a novel link between AHR and posttranscriptional regulation of WT1 isoform expression was characterized in this laboratory. The aryl hydrocarbon receptor (AHR) is an important ligand-activated nuclear transcription factor involved in the regulation of cellular differentiation. AHR-null mice exhibit systemic developmental deficits including nephropenia. Previous work has shown that exposure to environmental hydrocarbons that 
function as AHR ligands deregulate receptor signaling via proteasomal degradation and induce receptor-specific deregulation of WT1 mRNA splice variant ratios in murine metanephric organ and cell. This work led us to hypothesize that disruption of receptor signaling following loss of AHR protein results in WT1 dysregulation thereby altering expression of WT1 targets and renal cell differentiation genes. Evidence is presented here showing that AHR degradation mediated by small interfering RNA results in downregulation of $+\mathrm{KTS}$ and induction of the -KTS mRNA splice variants. WT1 dysregulation correlates with reductions in WT1 transcriptional targets (Synd1 Pax2, Egfr and Rara), as well as downstream markers of renal cell differentiation (Sfrp1, Igf1r, Igf2r, Wnt4, Lhx1, and E-cad). We conclude that AHR regulation of nephrogenesis occurs through preservation of WT1 mRNA isoform ratios within the kidney.

\section{Introduction}

The aryl hydrocarbon receptor (AHR) is a member of the basic helix loop helix Per ARNT Sim (bHLH-PAS) family of transcription factors and initially characterized for its role in the regulation of xenobiotic metabolizing enzymes (XMEs) necessary for removal of exogenous chemicals. While bHLH-PAS proteins share similar structure and function, the AHR is the only member of the family known to be regulated by ligand activation. Environmental chemicals such as polycyclic aromatic hydrocarbons act as AHR ligands leading to the 
dissociation of two molecules of the chaperone heat shock protein 90 (HSP90), $\mathrm{X}$-associated protein 2 (XAP2) and p32. The resulting conformational change in AHR exposes a nuclear localization sequence that facilitates nuclear translocation. In the nucleus, AHR dissociates from the chaperone complex and binds the aryl hydrocarbon receptor nuclear translocator (ARNT) resulting in association of the major groove DNA sequence containing 5'-GCGGGGGCG-3' and $(\mathrm{TCC})_{\mathrm{n}}$ repeats (Denison and Nagy, 2003). Translocation of the AHR-ligand complex to the nucleus can also trigger its proteolytic degradation regardless of ARNT binding (Roberts and Whitelaw, 1999). Depending upon cell type, AHR can be regulated by negative feedback inhibition in which the nuclear AHR complex activates transcription of the AHR repressor protein (AHRR) responsible for binding the xenobiotic response element (XRE) (Mimura et al., 1999). AHR transcriptional activity is regulated by association of complexes including AHRARNT, NF-kappa B, AP-1, and glucocorticoid receptor all of which, in turn, participate in numerous signaling pathways potentially relevant to nephrogenesis (Abbott et al., 1994; Hankinson, 2005; Hoffer et al., 1996; Miller et al., 2000; Tian et al., 2002).

Of relevance to this study are novel links identifying AHR as a regulator of the Wilms' tumor transcription factor (WT1), a master switch transcriptional regulator which initiates genetic events necessary for early nephrogenesis (Scharnhorst et al., 2001). Loss of the AHR through ligand activation, genetic mutation, or siRNA-mediated degradation results in dysregulation of WT1 mRNA 
isoforms and loss of renal cell differentiation markers (Falahatpisheh and Ramos, 2003; Falahatpisheh and Ramos, 2007; Nanez and Ramos, 2007). Studies using AHR -/- mice have led to findings that support the scenario in which AHR acts as an important nuclear transcription factor during embryogenesis and throughout maturity in multiple organisms. AHR -/- mice exhibit cardiac hypertrophy and elevated mean arterial pressure (Lund et al., 2003; Lund et al., 2006) that correlate to inductions in endothelin-1 and angiotensin II, known regulators of cardiomyocyte hypertrophy and hypertension (Gavras and Gavras, 2002; Yamazaki et al., 1996). Of relevance to renal biology is that angiotensin II is a potent renal vasoconstrictor that constricts efferent arterioles and increases blood pressure. Our laboratory has shown that AHR-null mice exhibit delayed nephrogenesis resulting in compromised renal development as evidenced by nephropenia and decreases in renal vasculature (Falahatpisheh and Ramos, 2003; McMillan and Bradfield, 2007b). Thus, it is possible that developmental renal deficits in AHR -/- mice stem from disruption of AHR signaling implicit in nephrogenesis.

Nephrogenesis proceeds in three morphologically distinguishable stages pronephros, mesonephros, and metanephros (Pohl et al., 2000). The precursor structure for renal development derives from the nephric ridge in the intermediate mesoderm. The pronephric duct gives rise to the pronephros which elongates to form the Wolffian duct. The Wolffian duct develops into the ureteric bud stimulating the mesonephric mesenchyme to undergo condensation and 
transdifferentiate from mesynchemal-to-epithelial cells. A cascade of genetic events regulated by proteins including WT1, glial cell derived neuronal factor (GDNF) and bone morphogenic protein-7, are responsible for regulating this process (Kuure et al., 2000). While homozygous deletion or mutation of either of these genes impairs nephrogenesis, the molecular mechanisms involved have yet to be fully characterized.

WT1 encodes a $\mathrm{Cis}_{2}-\mathrm{His}_{2}$ zinc fingered protein that functions via transcriptional regulation of target genes, including insulin-like growth factor II (Igf2r), syndecan-1 (Synd1), epidermal growth factor receptor (Egfr) and retinoic acid receptor- $\alpha(\operatorname{Rar} \alpha)$ in the early stages of renal development (Hosono et al., 1999). Homozygous WT1-/- mice do not develop normally, die in utero, and fail to differentiate into metanephric kidney from the existing pronephros (Kreidberg et al., 1993b) due to a failure of cells to undergo mesenchymal-to-epithelial differentiation (Avner, 1993). WT1 protein expression is strongest in the differentiating structures of the developing kidney; however, visceral epithelial cells (podocytes) are the only cell type that retains WT1 expression in mature kidneys.

Of importance to the present work is that WT1 activity is regulated in cis by different ratios of its own splice variants, or in trans by proteins such as bone marrow zinc finger 2 (BMZF2) or Pax2 (Lee et al., 2002). Perhaps the most studied regulatory mechanism involves the formation of WT1 +KTS/-KTS splice 
variants. KTS splice variants originate from the insertion of a lysine-threonineserine between the third and fourth zinc finger. The resulting change modifies the WT1 DNA binding specificity leading to activation and/or repression of target genes (Menke et al., 1998b). In humans, reduced WT1 +KTS mRNA isoforms result in severe kidney and gonad developmental deficits, collectively known as Frasier syndrome (Barbaux et al., 1997). Changes in exon 5 splice variants have also been associated with deficits in renal differentiation (Iben and Royer-Pokora, 1999). Addition of 17 amino acids in exon 5 creates an mRNA isoform that regulates transactivation (Wang et al., 1995). N-terminal residues 1-182 encode a dimerization region implicated in the regulatory mechanism exerted by dominant negative mutants (Englert et al., 1995; Reddy et al., 1995). We propose that disruption of AHR signaling resulting from receptor degradation deregulates WT1 resulting in an induction in the -KTS mRNA splice variant, thus altering the sequence of genetic events necessary for nephrogenesis.

Evolutionary comparison has shown that the $A h r$ is conserved across vertebrate and invertebrate species, many of which are not exposed to classical aromatic ligands (Hahn, 2002; Hahn et al., 2006). Evidence is mounting to support the idea that AHR functions as a key regulator of ontogenesis. In this study we demonstrate that degradation of the AHR results in dysregulation of WT mRNA splice variant ratios resulting in downregulation of direct genetic WT1 targets and renal cell differentiation markers. 


\section{Methods}

\section{Animals/Kidney Harvest}

C57BL/6J wild type mice were purchased from Jackson Laboratory (Bar Harbor, Maine) and placed under standard housing conditions. The care, breeding, and handling of animals were conducted in accord with NIH guidelines. Kidneys were resected, fixed in $4 \%$ paraformaldehyde, and embedded in paraffin.

\section{Immunohistochemistry}

WT1 (180) (Santa Cruz Biotechnology, Santa Cruz, CA) and AHR rabbit polyclonal antibodies (Biomol Research Laboratories Inc., Plymouth Meeting, PA) were applied overnight at $4^{\circ} \mathrm{C}$ in a solution containing $0.3 \%$ Triton $\mathrm{X}$ and $10 \%$ goat serum (Vector Laboratories, Burlingame, CA). Primary antibodly was detected using a goat anti-rabbit biotinylated secondary antibody (Molecular Probes), amplified with $A B C$ elite $(\mathbb{E})$, developed with diaminobenzidine (DAB) and 3, 3', 5, 5'-tetramethylbenzidine (TMB), respectively (Vector Laboratories, Burlingame, CA). Images were captured on a Zeiss Axiovert 300 microscope using Axiovision Rel 4.3 software interface (Carl Zeiss Inc.. Maple Grove, MN).

\section{Cell Cultures/ siRNA Transfection}


mK4 cells were kindly provided by Dr. Stephen Potter (Children's Hospital Medical Center, Cincinnati, OH). Cells were cultured in Dulbecco's modified Eagle's medium supplemented with $10 \%$ fetal bovine serum. Two different techniques were used for siRNA knockdown of AHR and WT1 targets. 1) mK4 cells were cultured and seeded at 30 cells $/ \mathrm{mm}^{2}$ in 6 well plates. Using DharmaFECT 2, mK4 cells were transfected with 150nM pooled siRNA AHR (ID\# L-044066-00-0005), WT1 (ID\# L040686-01-0005) or nonspecific sequence. After 4 days, cells were harvested for RNA isolation, cDNA synthesis, and protein extraction. 2) Hairpin siRNA was designed using verified Silencer Pre-designed siRNA directed towards AHR (ID\#188990) and WT1 (ID\# 100360). The resulting structures were cloned into a pSilencer 4.1 CMV hygro vector (Ambion, Austin, TX) and transfected into mK4 cells using Fugene 6. pSilencer 4.1 CMV hygro Negative Control (Ambion) was used to measure nonspecific effects. Stably transfected cells were selected for using $800 \mu \mathrm{g} / \mu$ l hygromycin over a 3 week period. Cells were harvested for RNA isolation, cDNA synthesis, and protein extraction.

\section{Protein Extraction and Western Analysis}

Total protein extraction was performed with M-PER reagent (Pierce Biotechnology, Rockford, IL) as described by the manufacturer. Protein concentration was normalized to bovine serum albumin and measured by Bradford analysis. Protein samples were heated at $70^{\circ} \mathrm{C}$ for 10 minutes and run on $4-12 \%$ NuPage $\AA$ Bis-Tris gels for one hour at 200 volts (Invitrogen, 
Carlsbad, CA). Proteins were transferred to PDVF membranes and probed with WT1 (180) (Santa Cruz Biotechnology, Santa Cruz, CA) and AHR rabbit polyclonal antibodies (Biomol Research Laboratories Inc., Plymouth Meeting, PA). Signal was visualized with secondary antibodies conjugated to horseradish peroxidase developed by SuperSignal $\circledast$ West Dura (Pierce Biotechnology, Rockford, IL). Values represent averages of at least three independent experiments.

\section{RNA Isolation and Real time PCR Amplification}

Total RNA was extracted from the cells using Trizol $($ according to manufacturer's specifications. Reverse transcription of RNA was carried out using iScript ${ }^{\mathrm{TM}}$ cDNA Synthesis Kit (Bio-Rad, Hercules, CA). Real time PCR amplification was performed using the iCycler Detection System (Bio-Rad, Hercules, CA). For each run, $25 \mu$ of $2 X$ SYBR Supermix (Bio-Rad, Hercules, CA) and $300 \mathrm{nM}$ for both forward and reverse primers in a total volume of $50 \mu \mathrm{l}$ were mixed. Quantitative PCR using primers specific for WT1 isoforms was performed to detect differences in the ratio of WT1splice variants ( $\left.{ }_{\mathrm{K}} \mathrm{KTS}\right)$ as follows. -KTS forward 5'-AGCTCAAAAGACACCAAAGGAG-3', reverse 5'GGGCTTTTCACCTGTATGAG-3'; +KTS reverse 5'-

GAAGGGCTTTTCACTTGTTTTAC-3'; -17aa 5'-CCTGAGGACGCCCTACAGC-3' reverse 5'-TGTGCCGTGGCCCTTTAAGG-3'; +17aa 5'CTGTGCCGTGGTTGCTCTGC-3'. All other primers were designed using 
Beacon Designer 5.1 to create amplicons from $150-300$ base pairs with an average melting temperature of $55^{\circ} \mathrm{C}$ depicted in Table 1.

Quantification was performed using comparative $\left(\Delta \mathrm{C}_{T}\right)$ method as described by Peinnequin et al. (2004). Briefly, the $\mathrm{C}_{\mathrm{T}}$ indicates the fractional cycle number for which the amount of amplified target reaches a fixed threshold. This amount is a constant depending on the primer set. The difference $\left(\Delta C_{T}\right)$ between the $\mathrm{C}_{T}$ of the target gene $\left(\mathrm{C}_{T_{\mathrm{t}}}\right)$ and the reference gene $\left(\mathrm{C}_{T_{\mathrm{r}}}\right)$ depends on the RNA relative copy number between the target and the reference gene. When the PCR have been properly optimized, the PCR efficiencies are close to one and the amount of target $\left(\mathrm{X}_{N}\right)$, normalized to an endogenous reference is given by the equation: $X_{N}=K \times 2^{-\Delta C T}$ were $K$ is a constant according to the target and reference primer sets (Lestaevel et al., 2003). The normalization to a calibrator (18S RNA) allows reduction of the previous equation by removing the constant $\mathrm{K}$. Under these conditions, the amount of target normalized to an endogenous reference and relative to a calibrator $\left(X_{N, C}\right)$ is given by the equation: $X_{N, C}=2^{-\triangle \Delta C T}$ where $\Delta \Delta \mathrm{C}_{\mathrm{T}}$ is the difference between the $\Delta \mathrm{C}_{\mathrm{T}}$ of the sample and the $\Delta \mathrm{C}_{\mathrm{T}}$ of the calibrator. The $\Delta \mathrm{C}_{T}$ method is based on i) similar amplification efficiency rates between target and reference genes and ii) PCR efficiency close to 1

\section{Matrigel Cell culture}

mK4 cells were cultured in Dulbecco's modified Eagle's medium supplemented with $10 \%$ fetal bovine serum and $5 \mathrm{X}$ mito serum extender (Becton 
Table 1: PCR Primers

Gene Forward Primer 5'-3'

\begin{tabular}{|c|c|c|}
\hline Gene & Forward Primer 5'-3' & Reverse Primer 5'-3' \\
\hline $18 \mathrm{~S}$ & CGTCTGCCCTATCAACTTTCG & GCCTGCTGCCTTCCTTGG \\
\hline Cyp1a1 & TCGTGTCAGTAGCCAATGTC & GCATCCAGGGAAGAGTTAGG \\
\hline Taurine Transporter & CATCCATCGTCATTGTGTC & AAGTTGGCAGTGCTAAGG \\
\hline Syndecan 1 & GAGAACAAGACTTCACCTTTG & AGCACTTCCTTCCTGTCC \\
\hline Paired box gene 2 (Pax2) & AGGTTTACATCTGGTCTGG & TAGGAAGGACGCTCAAAG \\
\hline $\begin{array}{l}\text { Epidermal growth factor } \\
\text { receptor (Egfr) }\end{array}$ & GAGGAGGAGAGGAGAACTG & GGTGGGCAGGTGTCTTTG \\
\hline $\begin{array}{c}\text { Retinoic acid receptor } \alpha \\
\text { (Rara) }\end{array}$ & CCCAGAAGACTAAAGTTGAC & TGGCAGGTAGTTGTGATG \\
\hline $\begin{array}{l}\text { Secreted frizzled-related } \\
\text { sequence protein } 1 \\
\text { (Sfrp1) }\end{array}$ & GCAGTTCTTCGGCTTCTA & ATGGAGGACACACGGTTG \\
\hline $\begin{array}{l}\text { Insulin-like growth factor I } \\
\text { receptor (lgf } 1 r)\end{array}$ & GTCCCTCAGGCTTCATCC & GAGCAGAAGTCACCGAATC \\
\hline $\begin{array}{c}\text { Insulin-like growth factor } 2 \\
\text { receptor }(\lg r 2 r)\end{array}$ & AGTATGTGAACGGCTCTG & TCTGTGATTGTCTGGATAGG \\
\hline $\begin{array}{l}\text { Wingless-related MMTV- } \\
\text { integration site } 4(\mathrm{Wnt} 4)\end{array}$ & GTAGCCTTCTCACAGTCCTTTG & GGTACAGCACGCCAGCAC \\
\hline $\begin{array}{l}\text { Lim homeobox protein } 1 \\
\text { (Lhx1) }\end{array}$ & ACCTAAGCAACAACTACAATC & AACACGGGAGTAGAAAGC \\
\hline E-Cadherin & CGACCCTGCCTCTGAATCC & CTTTGTTTCTTTGTCCCTGTTGG \\
\hline
\end{tabular}


Dickinson, San Jose, CA). Cells were seeded at $200 \mathrm{cells} / \mathrm{mm}^{2}$ in 6 well cell culture dishes coated with $500 \mu$ l of Matrige $(\circledR)$ (Becton Dickinson, San Jose, CA). Fresh media was added every three days. Renal bodies were harvested after two weeks for further analysis in situ.

Analysis of glomerular bodies was performed by seeding mK 4 cells in 6 well cell culture dishes coated with $100 \mu \mathrm{l}$ of Matrigel $($ in Dulbecco's modified Eagle's medium supplemented with $10 \%$ fetal bovine serum lacking $5 \mathrm{X}$ mito serum extender. After 4 days cultures were fixed in 50:50 Acetone methanol and processed for immunocytochemistry using WT1 rabbit polyclonal (180) and Thy1.1 mouse monoclonal antibodies visualized by goat anti rabbit 488 and goat anti-mouse 568 AlexaFluor (Molecular Probes-Invitrogen, Carlsbad, CA).

\section{WT1 expression vectors and selection}

pSRV plasmids containing all four WT1 splice variants from the 17aa/KTS sites including WT1A(-/-), WT1B(+//), WT1 $\mathrm{C}(-/+)$, and WT1D(+/+) were created as described by Haber et al. (Haber et al., 1992). WT1 sequences were excised with $\mathrm{Bgl} \mathrm{II}$ and inserted into p3XFLAG-CMV-14 expression vector (Sigma-Aldrich, St. Louis, MO). mK4 cells were transfected with $1 \mu \mathrm{g}$ of each splice variant and control vector. mK4 cells expressing vector derived gene products were selected for with Geneticin $®$ treatment (Invitrogen, Carlsbad, CA). WT1 splice variant overexpression was verified by QRT-PCR. 


\section{Results}

\section{AHR and WT1 colocalize in developing and adult glomeruli}

AHR protein is a ubiquitous protein expressed primarily in lung, thymus, liver, gut mucosa, kidney, and urogenital tissue (Jain et al., 1998).

Immunohistochemical (IHC) analysis revealed ubiquitous expression of the AHR throughout all parts of renal tissue with strongest signals occurring in glomeruli and endothelial cells of major renal vascular structures (Figures $1 \mathrm{~A}, \mathrm{~B}$, and C). Dual IHC analysis for AHR and WT1 was employed to determine cellular specificity and colocalization. In adult glomeruli, the strongest AHR signal colocalized with WT1 staining in the podocyte population (Figures 2A and B). In glomeruli of developing metanephros, AHR and WT1 colocalization occurs in the majority of the cell populations (Figures $1 \mathrm{C}$ and $\mathrm{D}$ ). This pattern is consistent with the known role for WT1 in mesenchymal-to-epithelial cell transition and the emerging role for AHR in renal development (Falahatpisheh and Ramos, 2003; Falahatpisheh and Ramos, 2007; Nanez and Ramos, 2007; Scharnhorst et al., 2001).

\section{siRNA-mediated Degradation of AHR Downregulates Renal Cell Differentiation Markers}

We have previously shown that exposure of metanephric organ cultures to $\mathrm{BaP}$, an environmental hydrocarbon that acts as an AHR ligand, inhibits nephrogenesis in a manner dependent upon AHR protein expression 
Figure 1. AHR expression in adult mouse kidney. AHR expression is highest in epithelial cells of renal vasculature and in glomerular podocytes. Immunohistochemical analysis of AHR protein as visualized by $3,3^{\prime}-$ diaminobenzidine (DAB) staining of formalin-fixed paraffin-embedded adult mouse kidney sections. In Panel A, AHR signal (brown color) localizes to the cells lining the renal vasculature. Panels $B$ and $C$ compare AHR and WT1 signal, respectively in adult glomeruli. Intense AHR signal (denoted by arrows) shows patterns indicative of podocyte expression when compared to WT1 signal. 
Figure 1
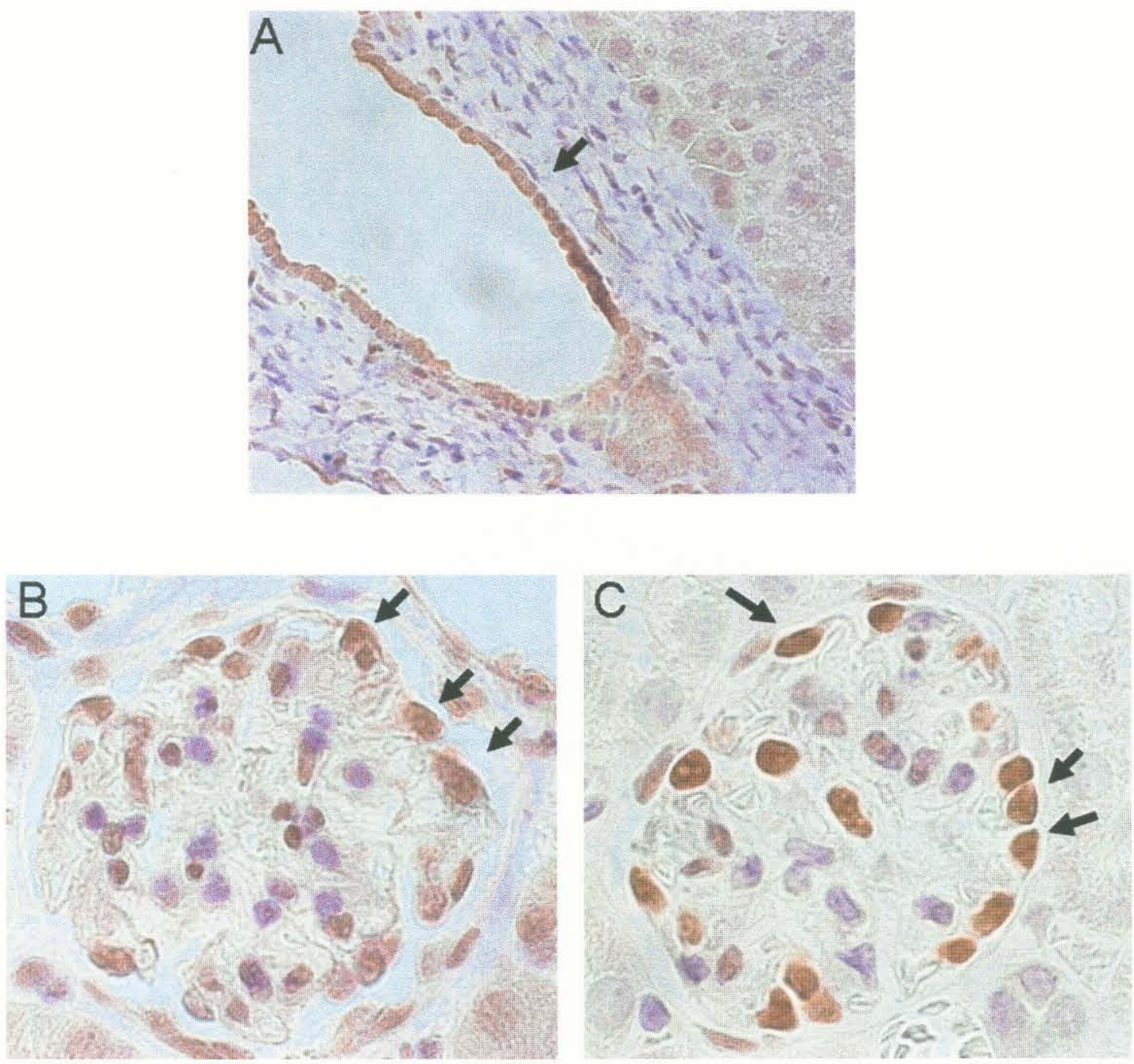
Figure 2. AHR colocalizes with WT1 expression. AHR and WT1 colocalize in the developing and adult kidney. Dual immunohistochemical analyses of AHR and WT1 proteins visualized using 3, 3', 5, 5'-tetramethylbenzidine (TMB) and NovaRED, respectively. Panels $A$ and $C$ are representative images taken of glomeruli from adult C57BL/6J mouse kidneys or E11 cultured metanephros cross-sections. In panel B, maximal AHR signal (blue color denoted by arrow) was measured using Zeiss Axiovision Rel 4.3 image analysis software. Positive AHR signal is outlined in red. AHR-WT1 colocalization was defined by a positive nuclear WT1 signal in proximity to the outlined AHR signal, as denoted by arrows. Because of the diffuse nature of the AHR signal throughout metanephroi in panel D, AHR-WT1 colocalization was determined by overlay of AHR and WT1signals. Normalized signal was always $<10 \%$ in sections stained separately with individual antibodies. Panels $E$ and $F$ are serial cross sections of C57BL/6J E11 cultured metanephroi analyzed for AHR alone or AHR-WT1 colocalization as described. The scale in panels A-D was $25 \mu \mathrm{m}$, and in panels $E$ and $F 100 \mu \mathrm{m}$. 
Figure 2
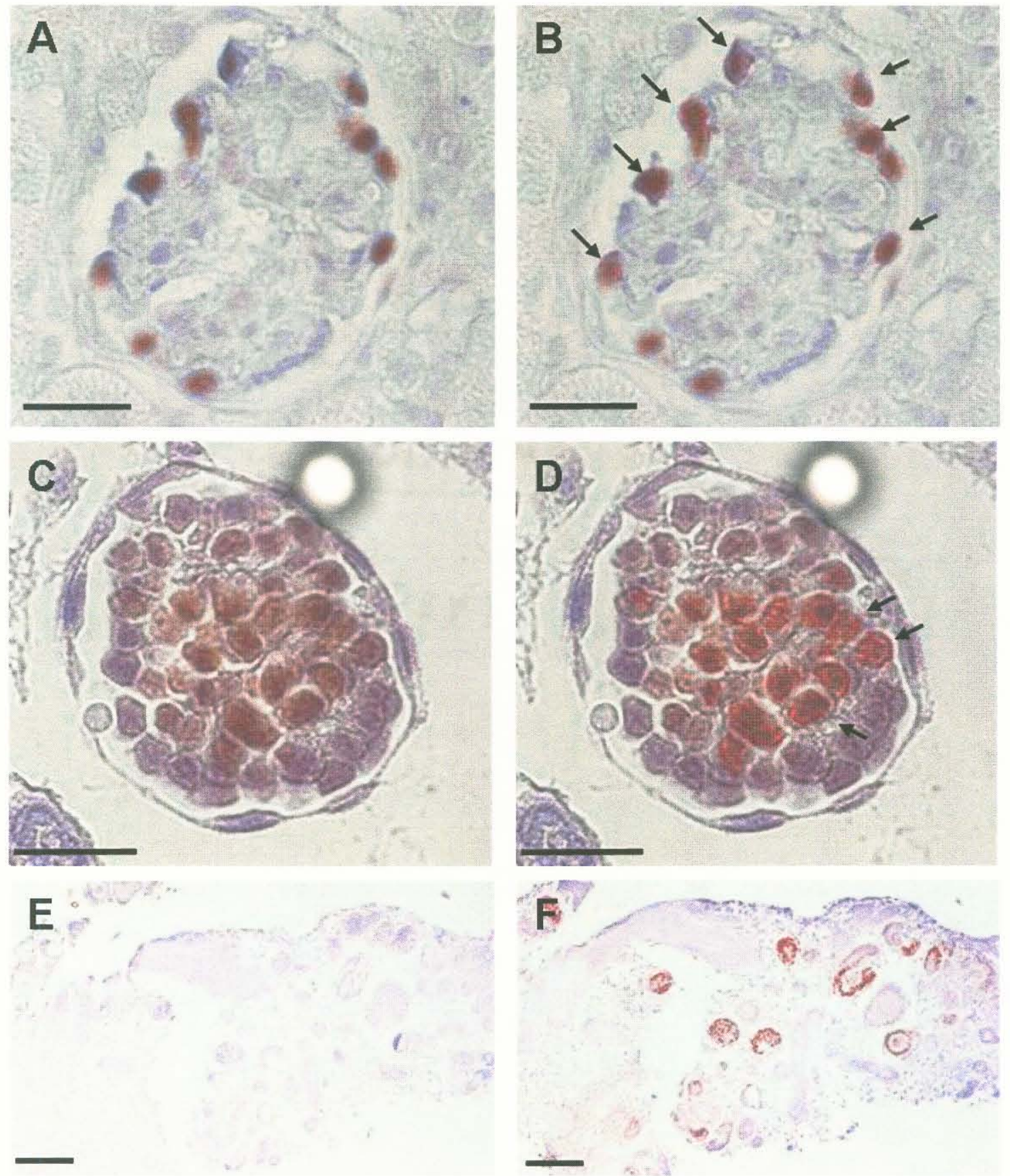
(Falahatpisheh and Ramos, 2003; Falahatpisheh and Ramos, 2007; Nanez and Ramos, 2007). In this model, BaP binds AHR resulting in nuclear translocation thus promoting transcription of AHRE containing xenobiotic metabolizing enzymes, and culminates in proteasomal degradation of AHR protein. To determine the effects of AHR degradation irrespective of ligand activation, mK4 cells of metanephric cell lineage were exposed to pooled siRNA directed against AHR. Seventy two hour siRNA exposure resulted in loss of AHR protein and mRNA (Figures $3 A, B$, and $C$ ). Exposure to AHR siRNA and subsequent loss of AHR protein resulted in downregulation of Igf1r, Wnt4, Igf2r, and E-cad as determined by quantitative RT-PCR (QRT-PCR) (Figure 3D). The mesenchymal marker Sfrp1 and Lhx1 did not change (Figure 3D). To determine if constitutive AHR degradation altered markers of renal cell differentiation, we examined mK4 cells expressing the pSilencer 4.1 CMV hygro vector containing a sequence that when transcribed produces a 19-mer hairpin with a loop and 3' terminal overhang that is processed by cellular machinery to produce siRNA (vsiRNA) (Paddison et al., 2002). After selection with hygromycin, cell populations expressing AHR vsiRNA exhibited reductions in AHR protein and mRNA (Figures $4 A, B$, and $C$ ). Constitutive AHR degradation resulted in decreased expression of Sfrp1, Igf1r, $\operatorname{lgf} 2 \mathrm{r}$, Lhx1 and E- cad mRNA (Figure 4D). However, unlike conventional siRNA, AHR vsiRNA resulted in an induction of Wnt4 and reductions in Sfrp1 and Lhx1. These differences may be due to separate functionally redundant transcriptional pathways activated after prolonged AHR degradation. 
Figure 3. siRNA-mediated degradation of AHR downregulates renal cell differentiation markers. $\mathrm{mK} 4$ cells were cultured and seeded at $30 \mathrm{cells} / \mathrm{mm}^{2}$ in the presence of $150 \mathrm{nM}$ pooled siRNA targeted for $\mathrm{AHR}$ or a nonspecific sequence for 3 days. Cells were processed for RNA isolation, cDNA synthesis, and protein extraction. Quantitative PCR was performed with values representing $\triangle \triangle C T$-normalized fold change relative to DMSO control (dashed line). Variance represents standard deviation between at least three biological replicates. Statistical significance was calculated using a Wilcoxon rank sum. In panels $A-C$, siRNA directed towards $A H R$ results in loss of $A H R$ protein and mRNA. In panel D, loss of AHR results in downregulation of Igf1r, Wnt4, Igf2r, and E-cad. 
Figure 3

A

AHR

$a-$ Tub

siRNA $\underset{\text { Non }}{\text { specific }}$ AHR WT1

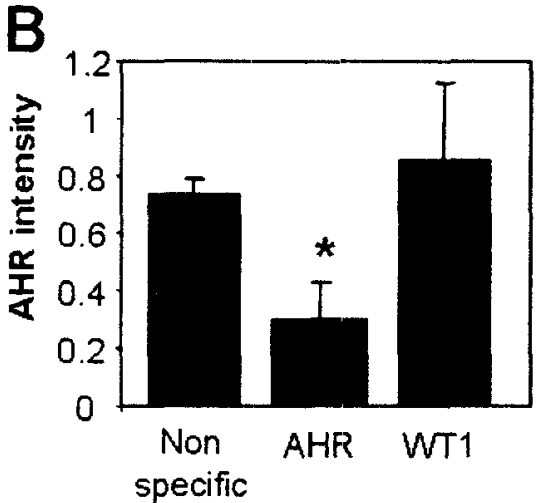

SIRINA

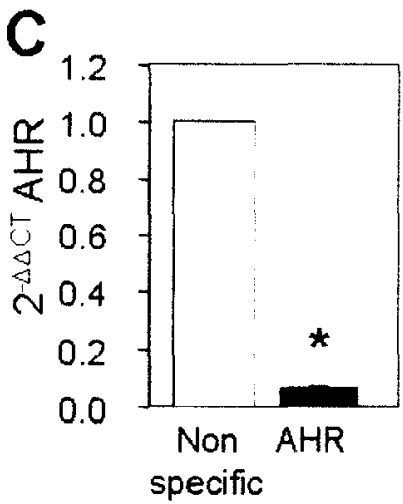

SiRNA

D 1.2

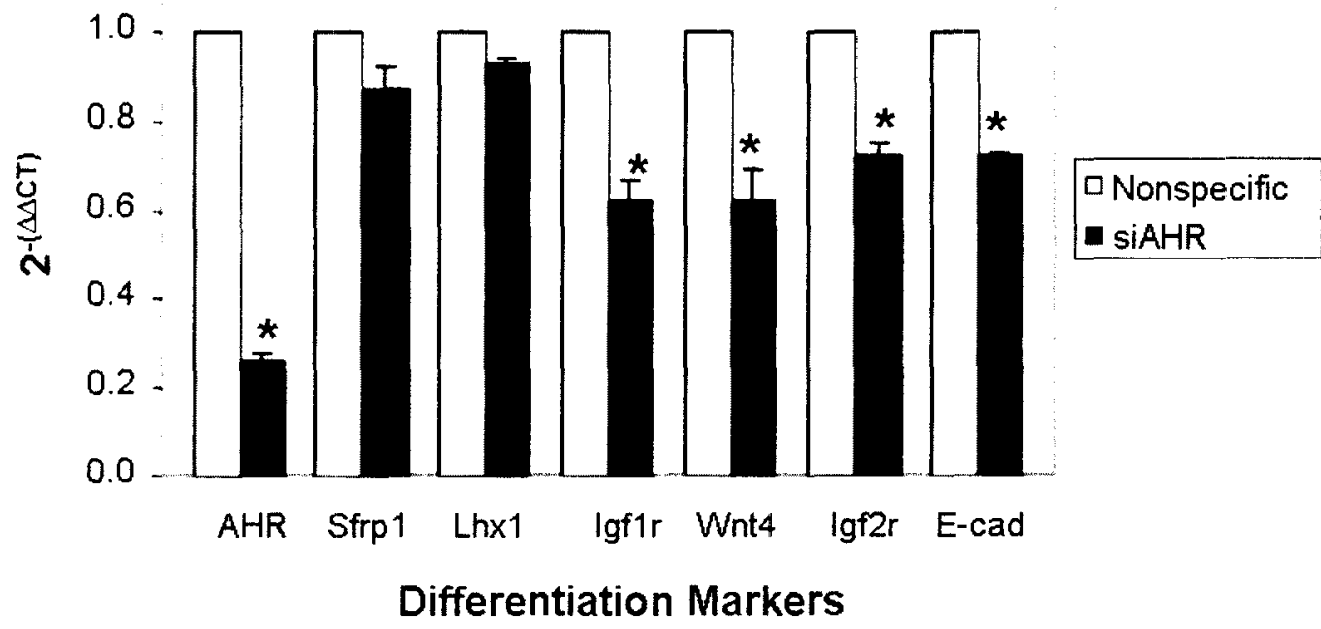


Figure 4. Vector-mediated siRNA degradation of AHR downregulates renal cell differentiation markers. $\mathrm{mK} 4$ cells were seeded at 50 cells $/ \mathrm{mm} 2$ and transfected with vectors containing siRNA sequences targeted for WT1 or a nonspecific. After two weeks selection with hygromycin, mK4 populations containing stable siRNA expression were processed for RNA isolation, cDNA synthesis, and protein extraction. Panel A and B, Western blot analysis of AHR protein normalized to a-tubulin loading control. siRNA directed towards AHR results in loss of AHR protein. Panel C and D, Quantitative PCR was performed with values representing $\Delta \Delta C T$-normalized fold change relative to DMSO control (dashed line). Variance represents standard deviation between at least three biological replicates. Statistical significance was calculated using a Wilcoxon rank sum. vsiRNA reduces Sfrp1, Igf1r, Igf2r, Lhx1, and E-cad. 
Figure 4

A

\title{
AHR
}

\author{
$a-$ Tub
}

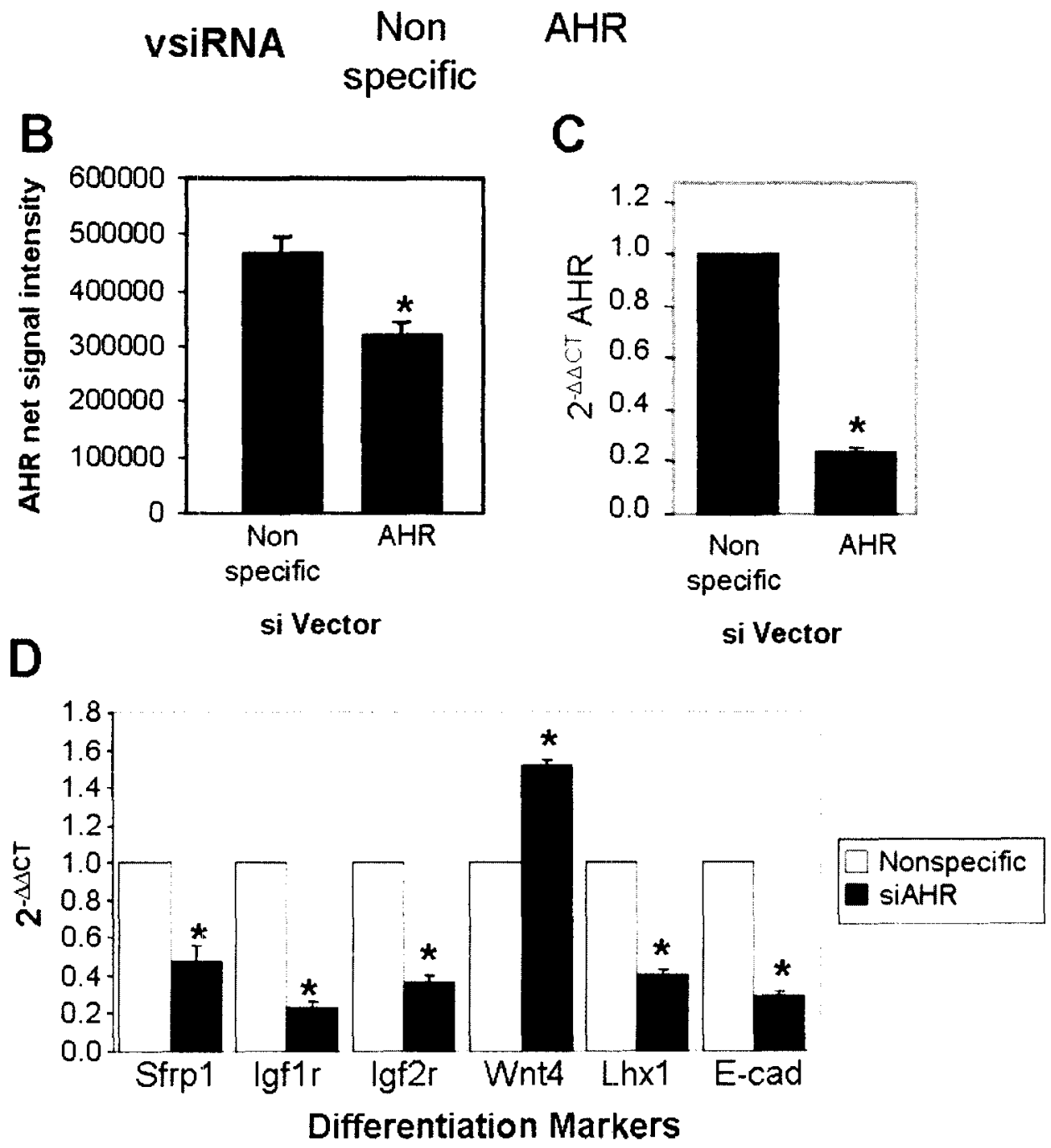


siRNA-mediated Degradation Induces -KTS WT1 mRNA and Downregulates

\section{Direct WT1 Transcriptional Targets}

Our next set of experiments examined whether AHR degradation was solely responsible for WT1 dysregulation. Using QRT-PCR, we measured relative amounts of -KTS and +KTS WT1mRNA, as well as, known WT1 transcriptional targets directly regulated by WT1 (Synd1 Pax2, Egfr, Rara, TauT) (Han and Chesney, 2003; Ryan et al., 1995; Saxen, 1987; Vainio et al., 1999). Both siRNA and vsiRNA-mediated AHR degradation resulted in an induction in KTS and reduction in +KTS (Figures $5 \mathrm{~A}, \mathrm{~B}, \mathrm{C}$, and D). In agreement with the known role of -KTS WT1 as a transcriptional suppressor (Menke et al., 1998a), AHR siRNA resulted in decreased expression of known targets Synd1, Pax2, Egfr, Rara, and TauT (Figure 5E). Constitutive vsiRNA-mediated AHR degradation resulted in selective downregulation of Synd1, Egfr, and TauT (Figure 5F). In contrast to siRNA, AHR vsiRNA did not alter Pax2 and induced Rara (Figure 5F). These results implicate a pathway whereby disruption of AHR signaling via protein degradation shifts WT1 mRNA isoform ratios towards -KTS resulting in functional changes in known WT1 transcriptional targets.

siRNA-mediated Degradation of WT1 Downregulates Direct Transcriptional Targets and Renal Cell Differentiation Markers

To compare the molecular consequences of elevated -KTS mRNA to loss of WT1 protein, mK4 cells exposed to WT1 siRNA were examined for mRNA expression of renal cell differentiation markers and known WT1 targets. WT1 
Figure 5. Loss of WT1 protein correlates with -KTS induction and target gene downregulation. In panels $\mathrm{A}, \mathrm{B}$, and $\mathrm{E}, \mathrm{mK} 4$ cells were cultured and seeded at 30 cells $/ \mathrm{mm}^{2}$ in the presence of $150 \mathrm{nM}$ pooled siRNA targeted for WT1 or a nonspecific sequence for 3 days. Cells were processed for RNA isolation and cDNA synthesis. Quantitative PCR was performed with values representing $\triangle \Delta C T$-normalized fold change relative to DMSO control. Variance represents standard deviation between at least three biological replicates. Statistical significance was calculated using a Wilcoxon rank sum. siRNA directed towards AHR resulted in -KTS induction and +KTS reductions that correlated to loss of WT1 target Synd1, Pax2, Egfr, Rara and TauT mRNA expression. In panels $\mathrm{C}, \mathrm{D}$, and $\mathrm{F}, \mathrm{mK} 4$ cells were transfected with vsiRNA directed towards AHR. vsiRNA selection resulted in cell populations with induced expression of -KTS and decreased expression of +KTS. WT1 targets Synd1, Egfr and TauT were downregulated while Pax2 remained unchanged and Rara was induced. 
Figure 5
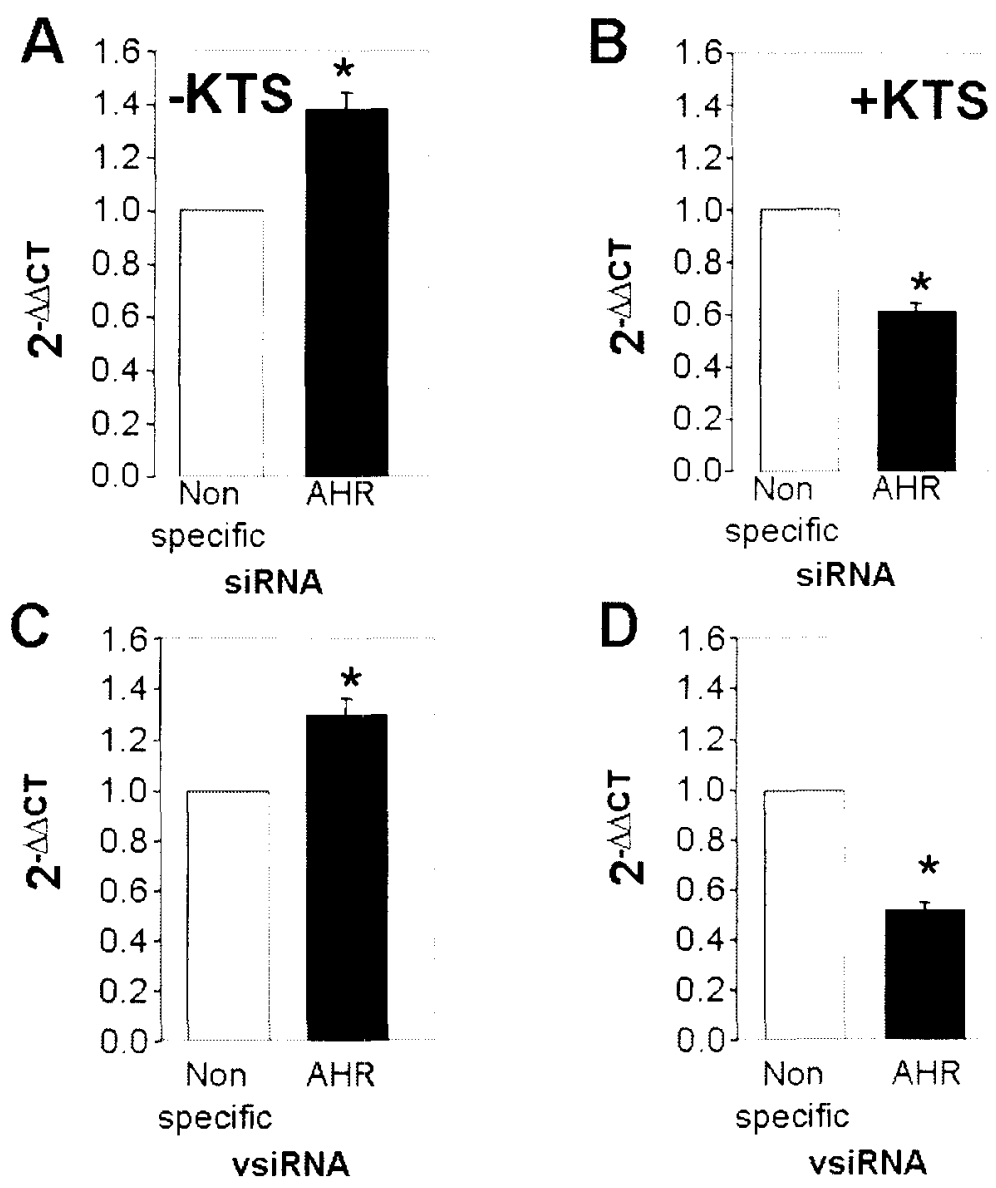
Figure 5
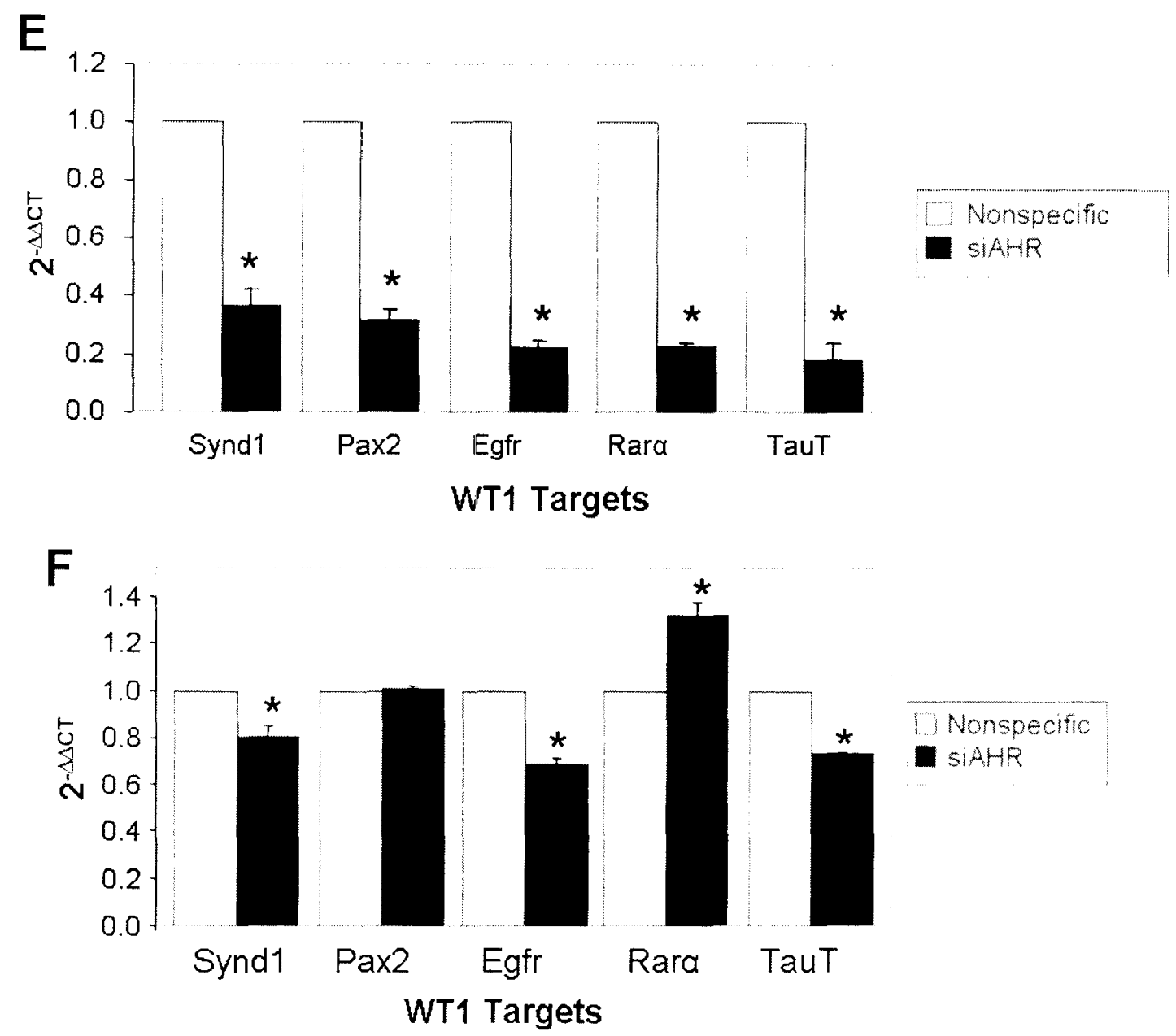
siRNA resulted in downregulation of WT1 protein and mRNA (Figures $6 \mathrm{~A}$ and $\mathrm{B}$ ). Similar to results seen with AHR siRNA-mediated -KTS induction, loss of WT1 protein resulted in downregulation of renal cell differentiation markers Sfrp1, Igf1r, Igf2r, Wnt4, and E-cad (Figure 6C). Lhx1 remained unchanged (Figure 6C). WT1 siRNA reduced mRNA expression of the WT1 transcriptional targets Synd1, Pax2, Egfr, and Rara (Figure 6D).

\section{Overexpression of WT1 mRNA Isoforms Downregulates WT1 Ttarget Genes}

To determine the effects of specific WT1 isoforms on WT1 target genes, cDNA sequences containing the four variants -17aa/-KTS (WT1A), -17aa/+KTS (WT1C), and +17aa/+KTS (WT1D) were cloned into a p3XFLAG-CMV-14 expression vector. mK4 cells stably expressing the vector were selected with G418 and assayed for molecular endpoints of WT1 and target gene expression. Figures $7 A-D$ shows confirmatory evidence that selected populations specifically expressed WT1A, WT1B, and WT1D mRNA isoforms in amounts significantly higher than empty vector. Compared to empty vector, expression of WT1A, C and D resulted in downregulation of Synd1, Pax2, Egfr, and Rara mRNA as evidences by QRT-PCR (Figures 7E and F). Although splice variant-specific expression of WT1 protein was not measured, the data indicate that the amounts of WT1 proteins produced by CMV promoter-driven overexpression are sufficient to downregulate WT1 targets, regardless of mRNA isoform. 
Figure 6. siRNA-mediated degradation of AHR downregulates renal cell differentiation markers. mK4 cells were cultured and seeded at $30 \mathrm{cells} / \mathrm{mm}^{2}$ in the presence of $150 \mathrm{nM}$ pooled siRNA targeted for WT1 or a nonspecific sequence for 3 days. Cells were processed for RNA isolation, cDNA synthesis, and protein extraction. Quantitative PCR was performed with values representing $\triangle \Delta C T$ normalized fold change relative to DMSO control (dashed line). Variance represents standard deviation between at least three biological replicates. Statistical significance was calculated using a Wilcoxon rank sum. In panels $A$ and $B$, siRNA directed towards AHR results in loss of AHR protein and mRNA. In panel C, WT1 siRNA results in downregulation of the differentiation markers Sfrp1, Igf1r, Igf2r, Wnt4, and E-cad. In panel D, WT1 siRNA results in downregulation of the WT1 targets Synd1, Pax2, Egfr, and Rara. 
Figure 6
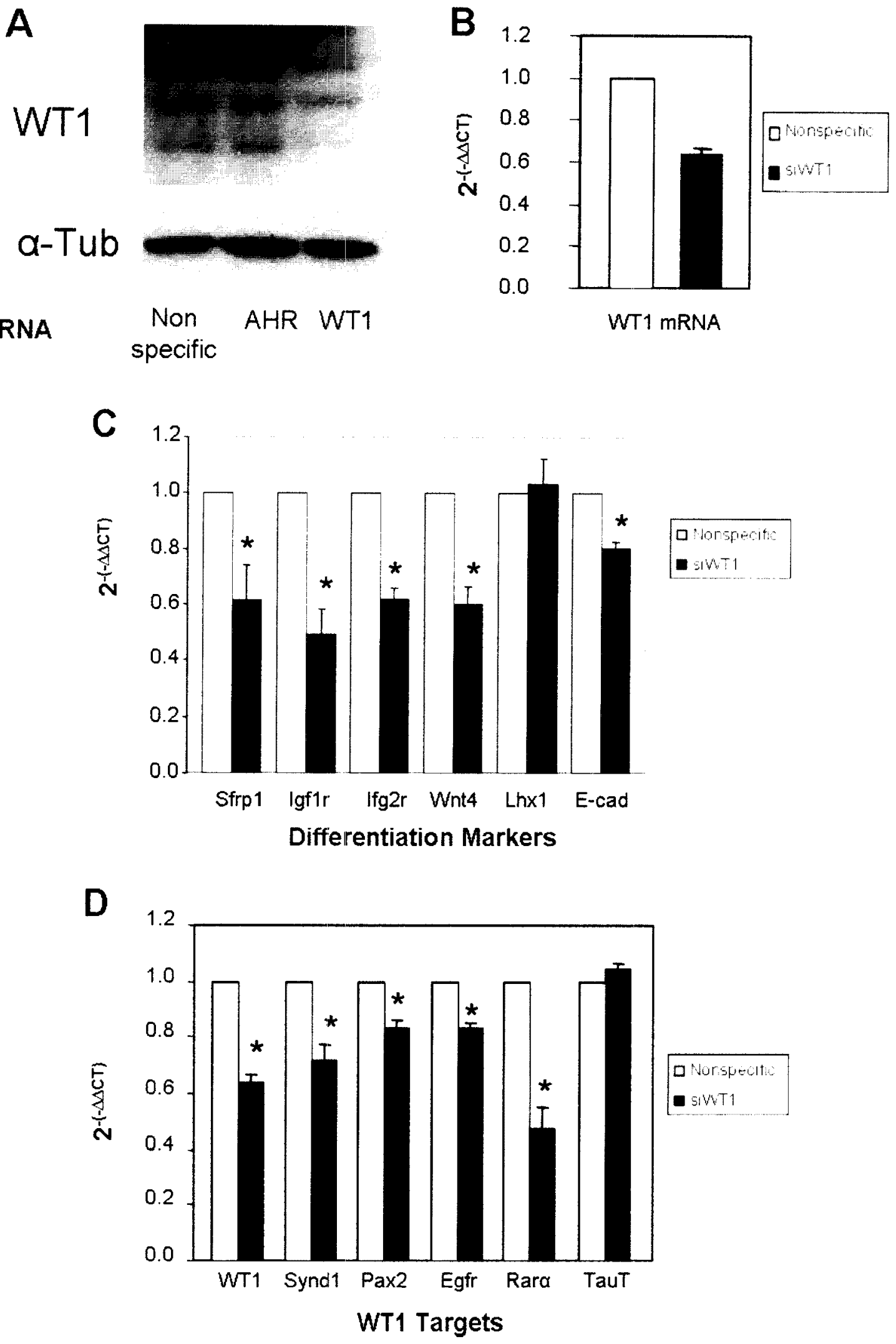
Figure 7. Overexpression of WT1 mRNA isoforms downregulates WT1 targets. P3XFLAG-CMV-14 expression vectors containing all four WT1 splice variants from the $17 \mathrm{aa} / \mathrm{KTS}$ sites including WT1A(-/-), WT1B $(+/-)$, WT1 $\mathrm{C}(-/+)$, and WT1D $(+/+)$ were transfected into mK4 cells. mK4 cells expressing vector derived gene products were selected by antibiotic resistance and examined for expression of WT1 splice variants by QRT-PCR, panels A, B, C, and D. Panels $E$ and $F$, overexpression of WT1 constructs downregulates WT1 targets Synd 1 , Pax2, Egfr, and Rara. 
Figure 7

A

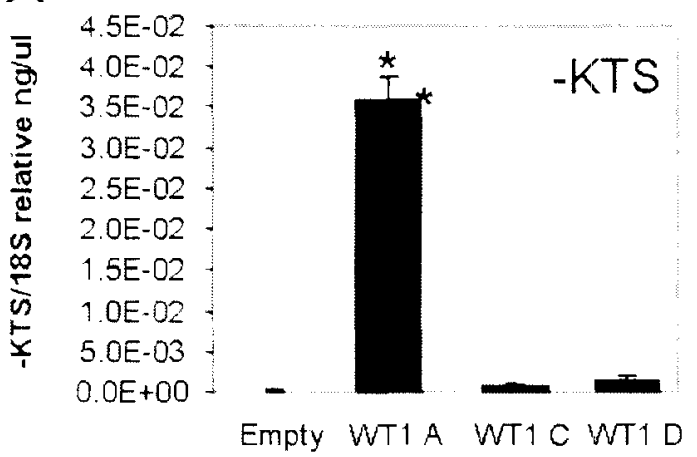

C

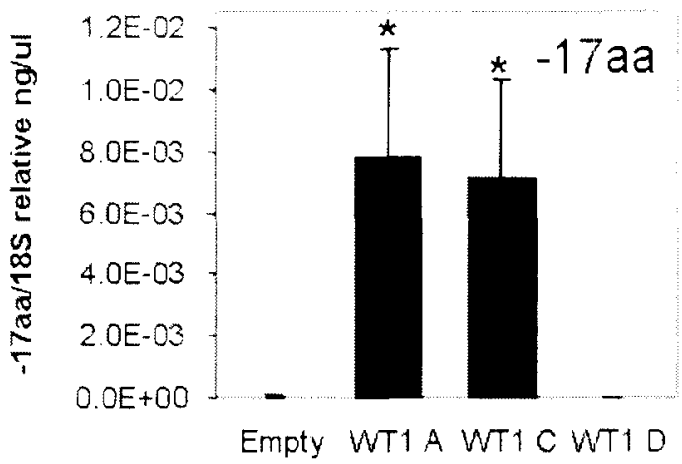

Vector

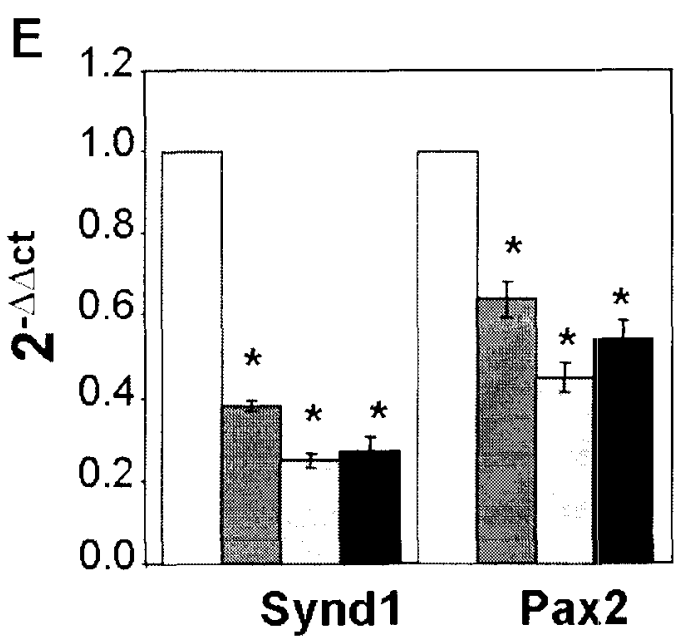

B

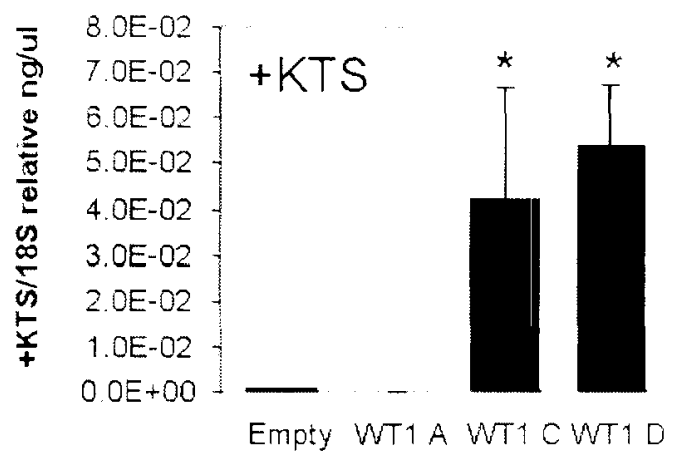

D

Vector
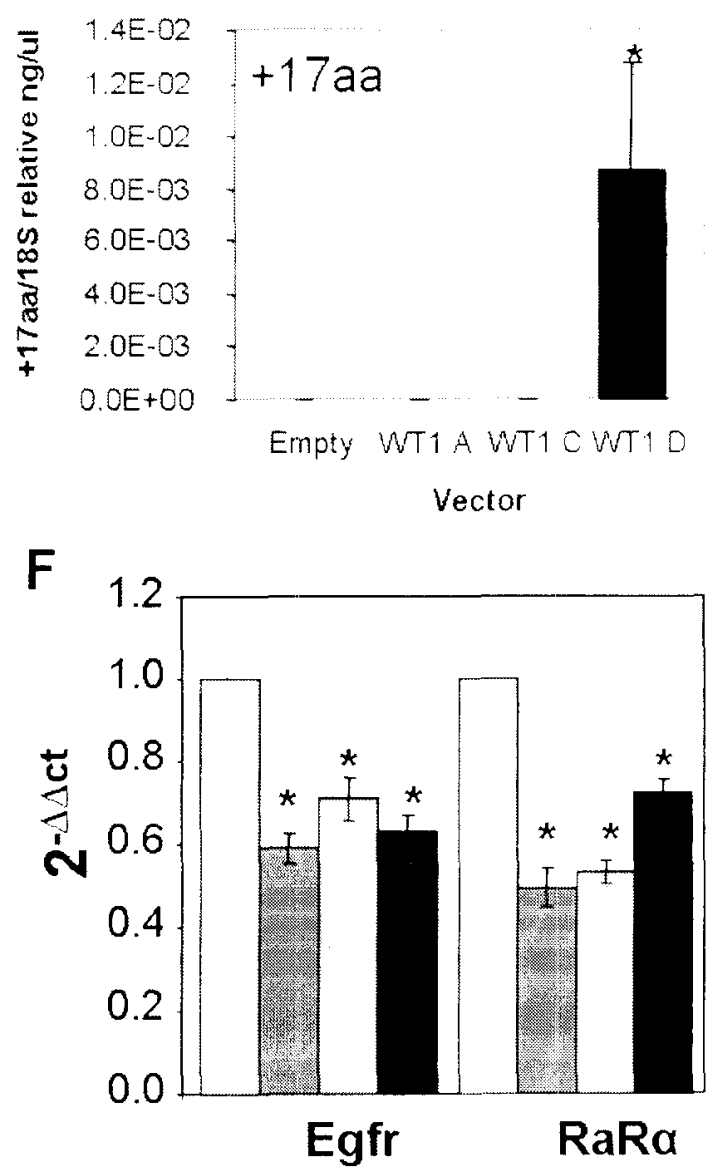


\section{Matrigel Induces Formation of Metanephric Structures in mK4 Cells}

To develop an in vitro model better-suited to examine the effects of WT1 splice variants on nephrogenesis, mK4 cells were seeded on Matrigel, a matrix known to induce differentiation and aggregation of various cell types into structures reminiscent of in vivo tissue ( for review see (Schmeichel and Bissell, 2003)). Culture of mouse mammary epithelial cells in matrigel promotes the formation of morphologically distinctive mammary alveoli which, in response to lactogenic hormones, produce milk (Li et al., 1999; Lin and Bissell, 1993; Sympson et al., 1994). The data shown in Figures $8 \mathrm{~A}-\mathrm{C}$ show that after 4 days in culture, mK4 cell cultures developed aggregates resembling metanephric bodies that closely resembled developing kidneys resected from E15 embryos (Figure 8D). Removal of mito serum extender from the media resulted in a typical cell culture monolayer with a ring of cellular growth that contained bodies morphologically resembling glomeruli (Figure 9A). Immuncytochemistry for markers specific for glomerular podocytes (WT1) and mesangial cells (Thy 1.1) revealed positive staining in glomerular structures (Figure 9B, C, and D). Collectively these data indicate that upon stimulation by matrix-related factors, nascent cell lines derived from metanephric cell cultures can provide a useful model for studying the molecular genetics of nephrogenesis. 
Figure 8. mK4 cells cultured on Matrigel form metanephric aggregates. mK4 cells were cultured in full media plus mito serum extender in 6 well cell culture dishes coated with $500 \mu \mathrm{l}$ of Matrigel $\otimes$. Panels A -C, metanephric renal bodies developed after 4 days of culture and showed morphological similarities to E15 metanephroi, panel D. 
Figure 8

A

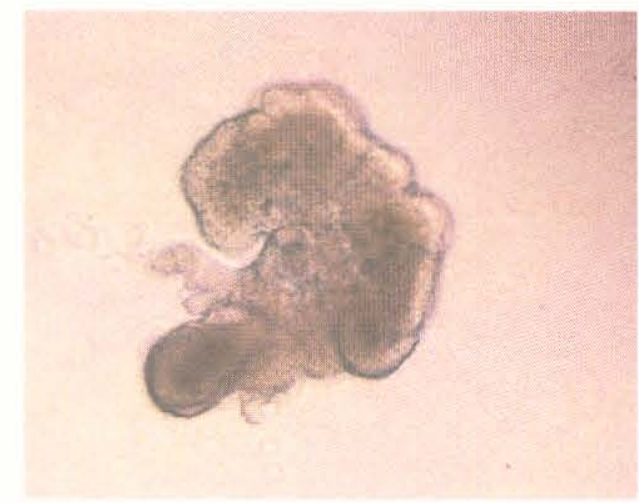

C

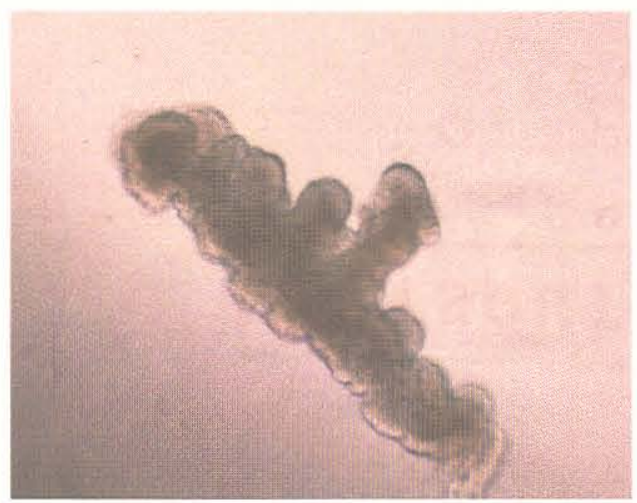

B

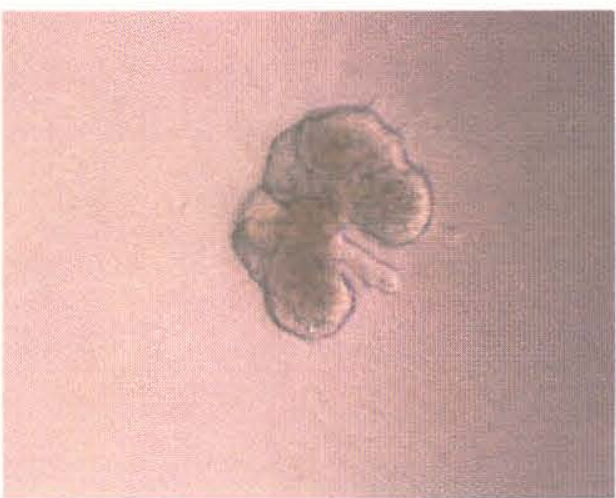

D

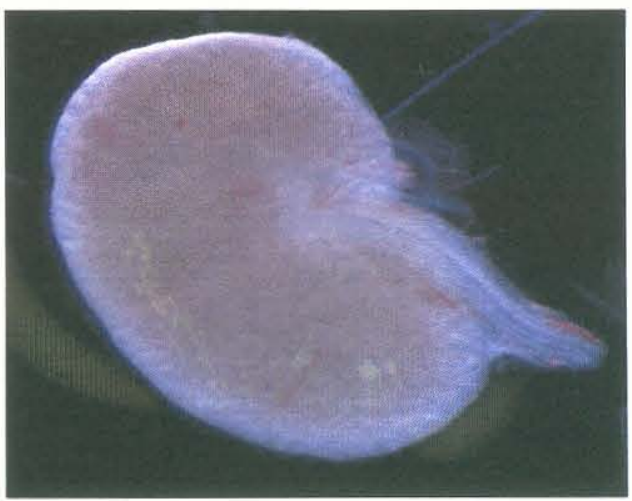


Figure 9. mK4 cells cultured on Matrigel form glomerular specific structures. mK4 cells were cultured in full media in 6 well cell culture dishes coated with $100 \mu$ l of Matrigel®. After 4 days, cultures were processed for immunocytochemical identification of glomerular markers. WT1 rabbit polyclonal (180) and Thy1.1 mouse monoclonal antibodies were visualized by goat antirabbit 488 and goat anti-mouse 568 AlexaFluor. Panel A, DAPI stained nuclei form a monolayer ring of cells around the outer portion of the cell culture well with morphologically visible glomeruli. Panel B, C, and D, WT1 signal (green) and Thy1.1 (red) colocalize in suspected glomerular structures. Arrows denote glomerular structures. 
Figure 9

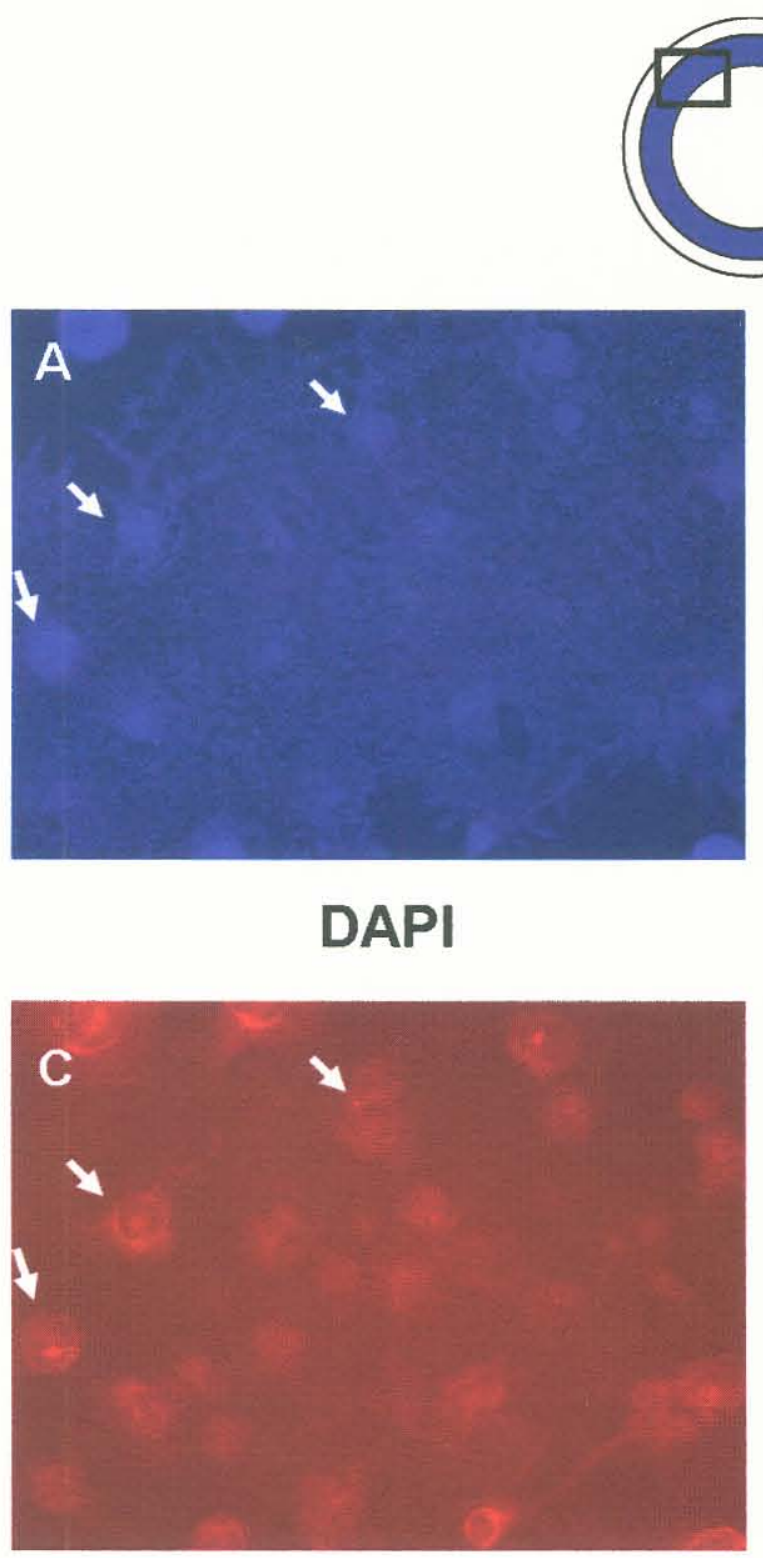

Thy1.1
Cell culture well

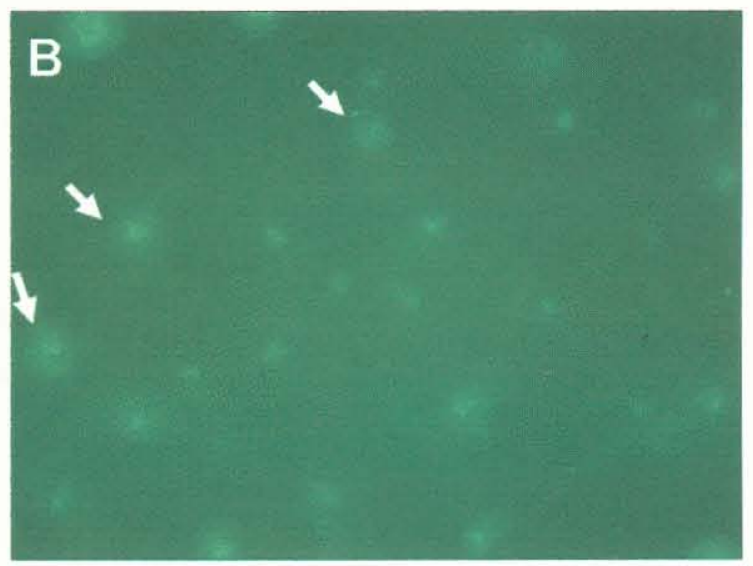

WT-1

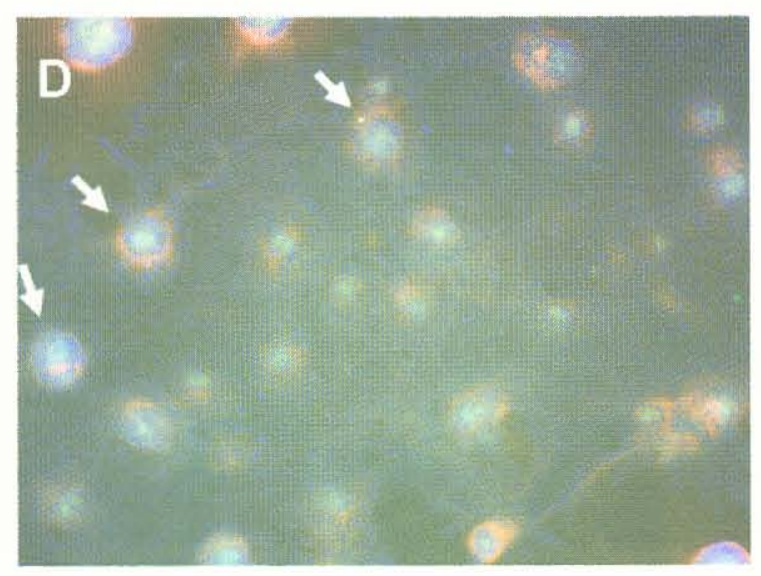

Merged 


\section{Discussion}

The emerging role for AHR in nephrogenesis is intriguing and emphasizes the need to understand the complexity of AHR signaling beyond xenobiotic metabolism (Falahatpisheh and Ramos, 2003; Fernandez-Salguero et al., 1995; Lahvis et al., 2000; Lahvis et al., 2005; McMillan and Bradfield, 2007b; Schmidt et al., 1996). Previous work in this laboratory has shown that exogenous ligands of the AHR inhibit nephrogenesis via a mechanism requiring conservation of AHR signaling and preservation of WT1 mRNA splice variant ratios and expression of renal cell differentiation markers (Falahatpisheh and Ramos, 2003; Falahatpisheh and Ramos, 2007; Nanez and Ramos, 2007).

In the present study we show that disruption of AHR signaling is linked to the protein degradation machinery of target cells in the developing kidney and results in disruption of genetic signaling implicit in nephrogenesis. AHR ubiquitination and degradation occurs both in the cytoplasm (Song and Pollenz, 2002), or upon nuclear translocation (Roberts and Whitelaw, 1999), and ARNT/DNA binding (Song and Pollenz, 2003). However, members of the proteasomal complex and the series of events leading to degradation have yet to be fully characterized. Recent work has shown that AHR functions as an E3 ligase and associates with proteasomal complexes consisting of DDB1, ARNT, TBL3 and CUL4B in the degradation of steroid sex receptors (Ohtake et al., 2007). Thus, it is likely that AHR participates in an auto-regulatory loop that 
promotes proteasomal degradation. In fact, the ubiquitin E3 ligase Itch, a family member of the Nedd4/Rsp5p HECT domain-containing family has been shown to participate in auto-ubiquitination facilitating its own proteasomal degradation (Mouchantaf et al., 2006).

siRNA-mediated reduction in AHR protein results in a decrease of renal markers of differentiation in agreement with pharmacological models of AHR degradation (Nanez and Ramos, 2007). This is the first known report indicating that a reduction in AHR protein is sufficient to disrupt expression of molecular markers necessary in nephrogenesis. Wnt4 is implicated in early nephron development and differentiation (Stark et al., 1994). Sfrp1, most likely through Wnt interactions, plays an important role in the mesenchymal to epithelial cell transition manifested during tubule formation and bud branching (Yoshino et al., 2001b). Lhx1-null mice do not progress past mesonephric development (Shawlot and Behringer, 1995a). Inhibition of the Igf1r impairs kidney growth, as evidenced by a reduction in nephrons and altered ureteric bud branching (Murphy and Barron, 1993; Wada et al., 1993). E-cad-null mice have decreased nephron number due to a failure of proper fusion of the metanephric mesenchyme to the ureteric bud (Mah et al., 2000). Conservation of AHR expression and constitutive signaling via expression of the nonresponsive $A H R^{d}$ allele, which does not degrade upon ligand binding, or treatment with antagonist, maintains expression of renal cell differentiation markers (Nanez and Ramos, 2007). These findings associate functional molecular consequences resulting 
from disruption of constitutive AHR functions with the regulation of renal cell differentiation.

We have shown that siRNA-mediated degradation of AHR results in induction of the -KTS WT1 mRNA splice variant. Depending upon cell type and stimulus, AHR functions in a diverse array of transcriptional complexes including the classical AHR-ARNT, NF-kappa B, A.P-1, and glucocorticoid receptor which provide possible mechanisms for regulation of WT1 splicing. Of these, the glucocorticoid receptor has been shown to associate with PGC-1, a known regulator of co-transcriptional splicing (Kinutti et al., 2001). Another possibility involves direct binding of AHR to the major groove of the WT1 promoter which contains two consensus AHR responsive elements within a regulatory region known to regulate transcription-coupled splicing events (Cohen et al., 1997) Determining the exact nature of AHR-WT1 promoter interactions and possible effects on splicing machinery directly (Bentley, 2002) or indirectly by altering elongation efficiency (Eperon et al., 1988; Roberts et al., 1998) remains an ongoing focus of the laboratory.

Our findings demonstrating induction in -KTS secondary to the loss of AHR are consistent with in vivo studies in which mice overexpressing the $-\mathrm{KTS}$ isoform have severely compromised renal development, evidenced by increased stromal tissue, decreases in tubular epithelium and glomerular tuft, and altered podocyte structure (Hammes et al., 2001). This is in agreement with previous 
work from the laboratory showing that in utero $\mathrm{BaP}$ exposure results in offspring with reduced glomerular number and glomerular filtration due to podocytopenia. While the exact molecular consequences resulting from alterations of the four abundant and biologically relevant isoforms of WT1 remains to be fully defined, it is well-established that the -KTS mRNA encodes a protein that participates in transcriptional activity, and that the protein encoded by +KTS mRNA associates with splicing factors (Davies et al., 1998; Hastie, 2001). In both metanephros and mK4 cell cultures, BaP-induced shifts in -KTS WT1 mRNA resulted in downregulation of differentiation markers, a finding consistent with the morphological deficits seen in -KTS transgenic mice and in our in utero exposure study. Ongoing studies will focus on the molecular consequences resulting from overexpression of each 17aa/KTS WT1 splice variant in the context of renal cell differentiation gene regulation.

Downregulation of WT1 protein did not induce WT1 targets for negative regulation. It is possible that $\sim 50 \%$ WT1 knockdown was not sufficient to ablate WT1 negative regulatory activity. Since $W T 1$ siRNA is not isoform specific, we assume that mRNA splice variant ratios were unchanged and that the negative regulatory role for $-\mathrm{KTS}$ is mediated more by the relative abundance of mRNA isoforms than by the total amount of protein expressed. Ongoing studies will use vector mediated WT1 siRNA to achieve more efficient degradation of specific variants. 
The development of an organ culture model system has been an important tool for discovery the molecular mechanism of ontogenesis. However incompatibility with assays necessary for further mechanistic discovery led us to examine alternative approaches. Extensive work carried out to recreate vasculature, kidney, lung, skin, and mammary tissue using 3D matrices has shown the promise and utility of 3D cultures in molecular biology (Sakurai et al. 1997; Schmeichel and Bissell, 2003; Schuger et al., 1990). Our model of Matrigel induced metanephric body formation agrees with the known role of extracellular matrices (ECM) from both the ureteric bud and the metanephric mesenchyme in ureteric branching morphogenesis. ECM protein expression is tightly regulated via growth factor signaling pathways which in turn are regulated by master switch transcription factors. Laminins compose the majority of the mature basement membrane, and have been shown to participate in branching morphogenesis and tubulogenesis (Engel, 1993). Exposure to anti-laminin5 blocking antibody inhibits renal development of cultured metanephroi by preventing association with $\alpha_{3} \beta_{1}$ and $\alpha_{6} \beta_{4}$-integrin receptors (Zent et al., 2001). In addition, cells stemming from the ureteric bud must digest ECM proteins to facilitate invasion. Both matrix metalloproteases (MMPs) and serine proteases are known to facilitate renal development. Membrane-type matrix 1 (MT-1-MMP) and MMP-2. oligonucleotide antisense inhibits metanephric development dependent on the TIMP-2 inhibitor activity (Kanwar et al., 1999; Ota et al., 1998). Thus, it is likely that renal morphogenesis requires elements secreted in the ECM to initiate biological events necessary for the initial and continued progression of 
nephrogenesis. The extent to which AHR participates in the regulation of cellmatrix interactions in the developing kidney remains to be established. Work by others has shown that expression regulates members of the MMP and laminin families (Hillegass et al., 2006) and we recently established a gene network where AHR interacts closely with osteopontin and other matrix-associated proteins (Johnson et al., 2004).

The present studies further define a novel role for AHR in the regulation of murine nephrogenesis. AHR expression is a key regulator of WT1 mRNA splice variant homeostasis. Loss of AHR results in dysregulation of WT1 manifest in inductions of -KTS and downregulation of WT1 targets and markers of renal cell differentiation. The matrix-dependent stimulation of mK4 cells into metanephric tissue may provide a useful model for examination of the molecular events responsible for nephrogenesis. 


\section{CHAPTER 5}

\section{DISCUSSION}

Although WT1 has been widely studied with respect to renal development and oncogenesis, many questions remain unanswered. The majority of work to date has focused on putative WT1 targets and correlations between splice variant expression and clinical outcomes in vivo (for review see (Niaudet and Gubler, 2006)). A major gap in our knowledge is the limited amount of information available about the putative factors that regulate splicing. Our work demonstrating that loss of AHR expression results in -KTS mRNA induction suggests AHR as putative regulator of WT1 mRNA splicing. This is in agreement with our in utero studies where BaP exposure results in offspring with reduced glomerular number and glomerular filtration due to podocytopenia. While the exact molecular consequences resulting from alterations of the four abundant and biologically relevant isoforms of WT1 remains to be fully defined, it is wellestablished that the -KTS mRNA encodes a protein that participates in transcriptional activity, and that protein encoding +KTS mRNA associates with splicing factors (Davies et al., 1998; Hastie, 2001). In both metanephros and mK4 cell cultures, BaP-induced shifts in -KTS resulted in downregulation of differentiation markers, a finding consistent with the morphological deficits seen 
in -KTS transgenic mice and in our in utero exposure study. Ongoing work will utilize constructs of all four KTS/17aa WT1 mRNA splice variants expressed in cells of varying differentiation status to begin defining the mechanism by which WT1 regulates the genetic cascade necessary for renal development. Our data implicate the participation of AHR signaling in WT1-mediated regulation of nephrogenesis.

Of direct importance to our work is the emerging role of AHR in renal development. Studies have shown roles for AHR in cell proliferation, adhesion and migration, all processes necessary for embryogenesis (for reviews see (Barouki et al., 2007; Ohtake et al., 2007)). The Ramos laboratory has shown that AHR-/- mice exhibit delayed nephrogenesis resulting in compromised renal development as evidenced by nephropenia and decreases in renal vasculature (Figure 1) (Falahatpisheh and Ramos, 2003; McMillan and Bradfield, 2007b). We have also shown that these changes can be reproduced by proteasomal decradation of AHR protein upon ligand binding. While our work has chiefly focused on WT1 dysregulation at the posttranscriptional level as a key mechanism by which AHR exerts regulatory influences, little is known about other possible mechanisms that may contribute to deficits in nephrogenesis, such as direct transcriptional regulation of renal cell differentiation markers or participation in proteasomal degradation of renal regulatory proteins (Figure 2).

A major theme of this dissertation revolves around the developmental deficits associated with disruption of WT1 signaling and differentiation resulting 
Figure 1. AHR-/-mice exhibit deficits in renal development with consequences in adult kidney morphology. Hematoxylin and Eosin stain of developing mouse metanephros shows that AHR-/- have less developed embryonic kidneys. Latex cast of AHR-/-adult kidneys have less vascularization compared to wild type mice. 
Figure 1
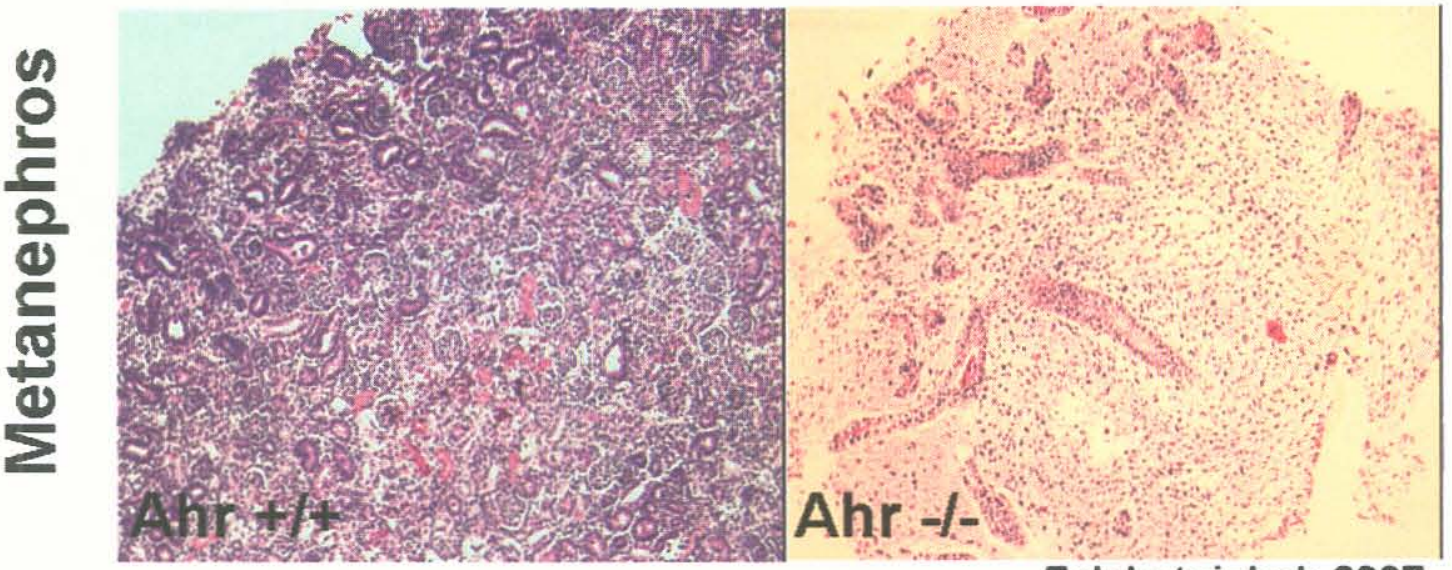

Falahatpisheh 2007

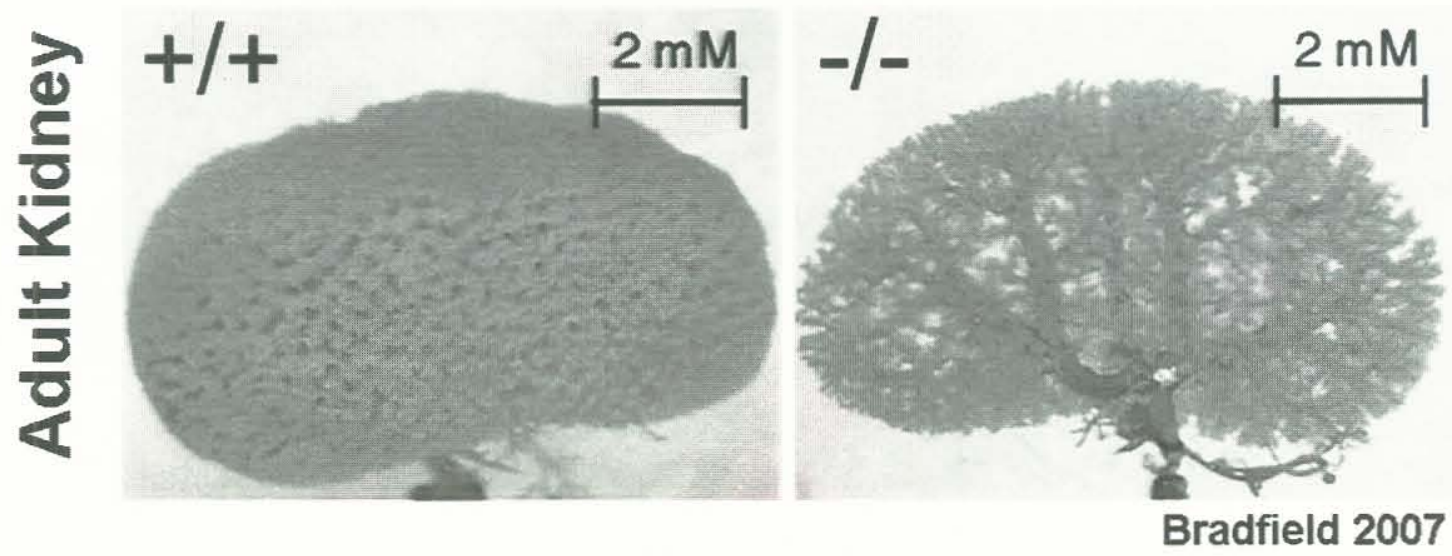


Figure 2. Schematic detailing the complexity of possible mechanisms by which Ahr may regulate WT1. Transcriptional activity may directly regulate splicing factors. AHR may participate in proteolytic degradation of factors implicit in WT1 splicing. 
Figure 2

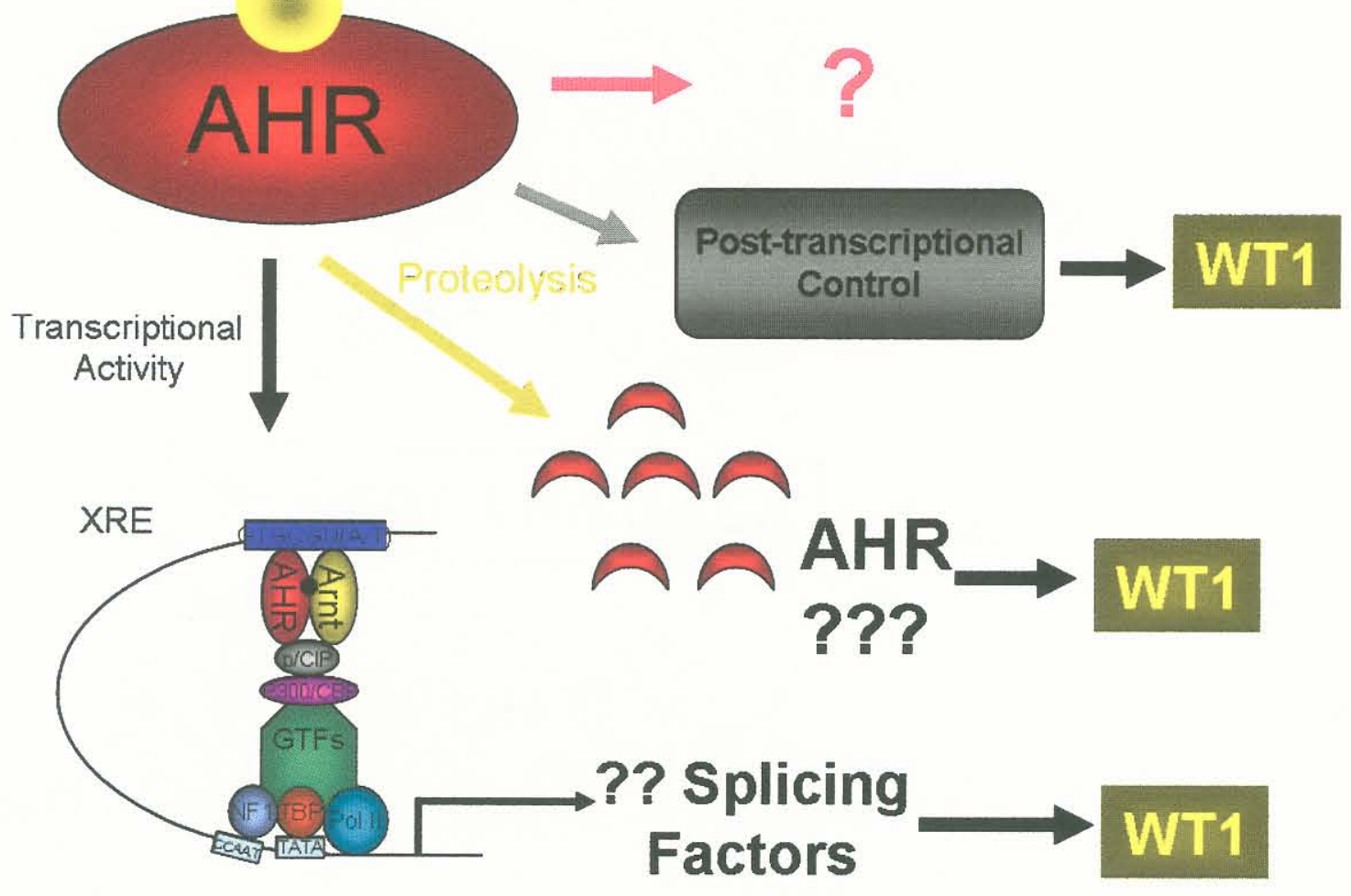


from the loss of AHR protein. In our earlier work, exposure of wild type metanephros to BaP leading to nephrogenic deficits similar to those seen in untreated AHR-/- metanephros implicated AHR as a regulator of renal development (Falahatpisheh et al., 2005). In agreement with known renal deficits in AHR-/- mice, Chapters 1 and 2 used in vivo and in vitro models to show that loss of AHR protein expression correlated with the downregulation of renal cell differentiation markers, and WT1 dysregulation. This is the first report showing that loss of AHR protein, regardless of biological context following ligand binding, is sufficient to disrupt expression of molecular markers implicit in nephrogenesis. To date, most studies implicating AHR in the development of liver, cardiovascular, and reproductive organs have shown strong correlations for AHR involvement, but lack any direct evidence of interactions with downstream effectors responsible for disruption of organ development (Fernandez-Salguero et al., 1995; Lahvis et al., 2000; Lahvis et al., 2005; Lund et al., 2003; Lund et al., 2006; Schmidt et al., 1996). Our discovery of AHR-dependent regulation of WT1 a known renal master switch transcription factor, is the first study to propose a direct mechanism for the role of AHR in ontogenesis (Figure 3).

A theme consistent in all of our studies is that loss of the AHR induced by $\mathrm{BaP}, \mathrm{TCDD}$, siRNA AHR exposure, or genetic manipulation in AHR-/- mice (Falahatpisheh and Ramos, 2007) results in the downregulation of known markers of renal cell differentiation Sfrp1, Lhx1, Igf1r, Igf2r, Wnt4, and E-cad (Barnes et al., 1994; Cho et al., 1998; Dong Van Huyen et al., 2003; Stark et al., 
Figure 3. Conceptual framework for AHR regulation of nephrogenesis.

Constitutive AHR signaling regulates WT1 function allowing for normal nephrogenesis. Exogenous AHR ligands in the form of PAHs initiates a response that results in WT1 dysregulation as evidenced by changes in the amounts of mRNA splice variants that ultimately disrupt the cascade of genetic events responsible for nephrogenesis 
Figure 3
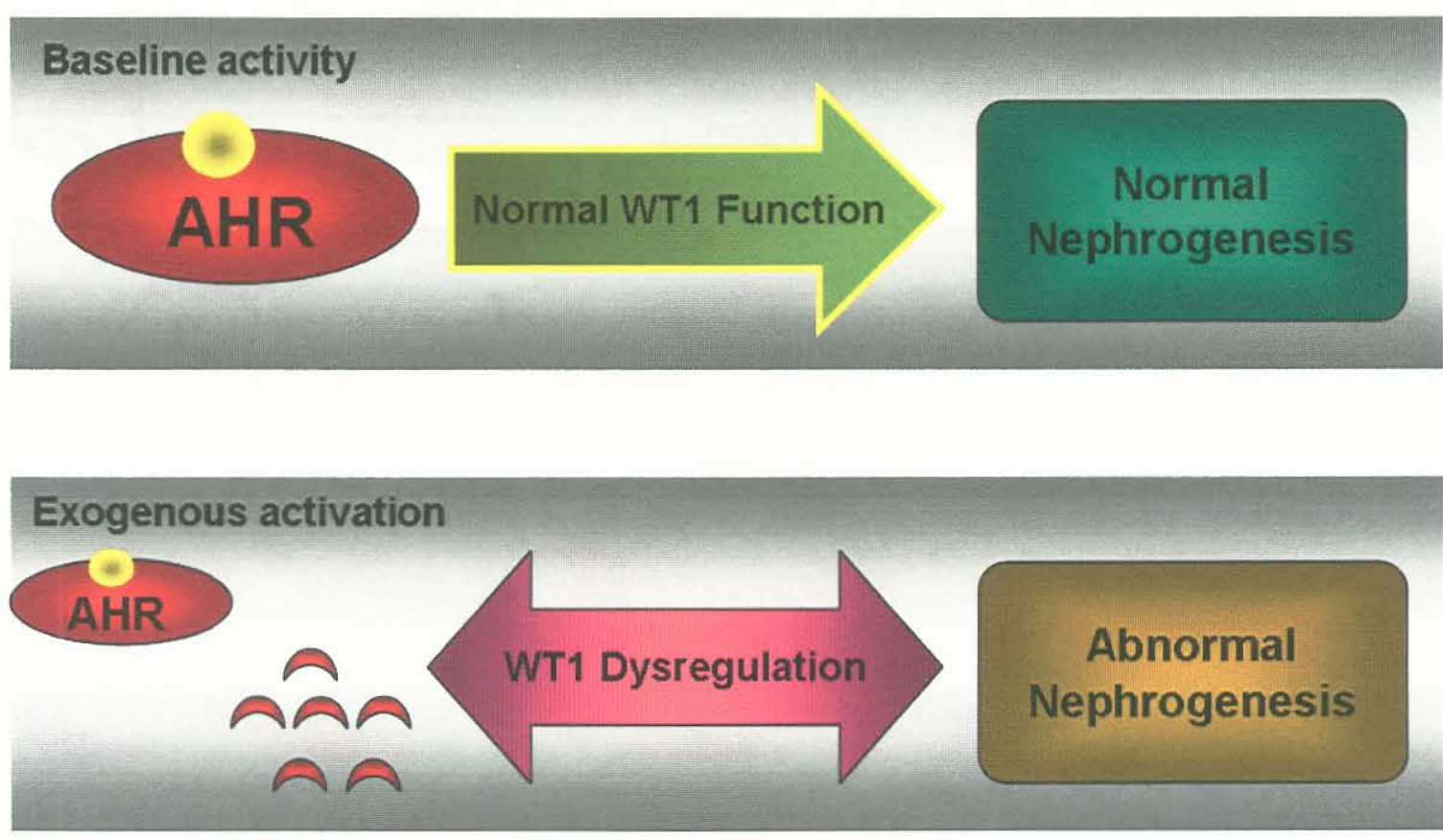
1994; Vainio et al., 1989; Yoshino et al., 2001b). While the involvement of WT1 in the regulation of targets such as Wnt4 (Sim et al., 2002) have been previously made, the mechanisms by which loss of AHR protein downregulates target gene expression remain unknown. Wnt4 is implicated in early nephron development and differentiation, and has been identified as a possible regulator of AHR transcription in prostate cancer cells (Chesire et al., 2004; Stark et al., 1994). This suggests a possible scenario in which loss of AHR results in WT1 dysregulation and subsequent downregulation of Wnt expression leading to generalized disruption of protranscriptional signals derived from Wnt/beta-catenin signaling and further loss of AHR protein. Sfrp1, through Wnt antagonistic interactions, plays an important role in the mesenchymal-to-epithelial cell transition during tubule formation and bud branching. Sfrp1 contains AHRRE elements in the regulatory region, suggesting the AHR may also regulate $\mathrm{Wnt}$ signaling via Sfrp1 transcriptional control (Yoshino et al., 2001b). In a similar manner, the inhibition of the Igf1r impairs kidney growth, as evidenced by a reduction in nephrons and altered ureteric bud branching (Murphy and Barron, 1993; Wada et al., 1993). Activation of the Igf1 $\mathrm{r}$ necessitates binding of extracellular insulin growth factors (IGF), a process that is tightly regulated by a family of proteins named insulin growth factor binding protein (IGFBP). AHR is a known regulator of IGFBP expression and may alter Igf1r activity through induction of IGFBP proteins (Johnson et al., 2004; Marchand et al., 2005). Perhaps the best marker of renal epithelial cell differentiation is E-cad as transition from mesenchymal to epithelium results in increased expression of this 
critical epithelial gene (Cho et al., 1998). E-cad-null mice have decreased nephron number due to a failure of proper fusion of the metanephric mesenchyme to the ureteric bud (Mah et al., 2000). Snail binds to the E-cad promoter repressing mRNA and protein expression (Nieto, 2002). Depending on cell type, snail expression can be repressed by modulators including fibroblast growth factor (FGF) releasing inhibitory control of E-cad and inducing mesenchymal-to-epithelial cell transition (Nieto, 2002). Of relevance to our work are studies identifying AHR as a transcriptional inducer of snail/slug family members (Ikuta and Kawajiri, 2006). In addition to direct regulation via WT1 (Hosono et al., 2000), it is possible that AHR signaling may regulate E-cad expression through snail/slug dependent pathways. While these markers have yet to be fully examined in AHR-null mice, the preservation of AHR expression and constitutive signaling via expression of $A H R^{d}$ or treatment with antagonist, restores their expression. In conclusion, the diverse mechanistic possibilities for AHR regulation of renal cell differentiation markers further highlights the importance of conservation of AHR signaling in renal cell differentiation and development.

The work presented in this study identified several novel roles for the $\mathrm{AHR}$, in addition to its well-established functions as a regulator of $\mathrm{XME}$. Evidence compiled from studies using AHR-/- mice, hydrocarbon exposure, and nonmammalian AHR homologues has implicated AHR as an early regulator of mammalian development (Falahatpisheh and Ramos, 2003; Falahatpisheh and 
Ramos, 2007; McMillan and Bradfield, 2007b; Nanez and Ramos, 2007; Puga et al., 2005). Expression of AHR is conserved across vertebrate and invertebrate species (Hahn, 2002; Hahn et al., 2006) with developmental functions ranging from limb growth to neuronal development (for review (Barouki et al., 2007)). The AHR homologue in Drosophila melanogaster Spineless (ss) regulates leg structures through both transformation and deletion mechanisms and regulates bristle size (Duncan et al., 1998). Loss of AHR1 in the Caenorhabditis elegans ortholog of AHR, results in cell axonal migration deficits resulting in impaired neuronal differentiation and function (Duncan et al., 1998; Qin et al., 2006). AHR-/- mice exhibit deficits in vascular structures stemming from a failure of the ductus venosus to close and result in reduced liver weight, microvesicular fatty metamorphosis, prolonged extramedullary hematopoeisis, and portal hypercellularity (Fernandez-Salguero et al., 1995; Lahvis et al., 2000; Lahvis et al., 2005; Schmidt et al., 1996). Of direct relevance to renal function are experiments in which AHR -/-mice were found to have cardiac hypertrophy and elevated mean arterial pressure (Lund et al., 2003; Lund et al., 2006). Hypertension correlated to inductions in endothelin-1 and angiotensin II, known regulators of cardiomyocyte hypertrophy (Gavras and Gavras, 2002; Yamazaki et al., 1996).

In studies of fetal TCDD exposure, a PAH that exerts its biological effects solely through AHR disruption, wild type mice exhibited cleft palate, hydronephrosis, small kidneys, and tortuous ureters (Peters et al., 1999b). 
TCDD-induced fetal deficits were shown to be regulated by transcriptional activation as mice expressing AHR with a mutated bHLH region (AHRbHLH-/-) were protected (Peters et al., 1999b). This study failed to characterize AHR expression which is likely regulating TCDD-induced developmental deficits. Thus, it is likely that in wild type mice TCDD activates AHR resulting in proteasomal degradation, disruption of AHR signaling and fetal programming. However in AHRbHLH-I- mice, TCDD exposure does not result in transcriptional activation, a key step in ligand mediated AHR degradation, thus conserving AHR protein expression and constitutive signaling. These results support our own studies in which siRNA-mediated AHR degradation alters expression of differentiation markers and deregulates WT1 activity as well as data from AHR-/mice (Falahatpisheh et al., 2007). Further evaluations using alternative mouse models of varying AHR genotype such as D2N mice with nonresponsive ligand binding $A H R^{d}$, or AHR-/- null mice lacking AHR protein, will lend further insight to this hypothesis.

The work presented in this study is the first report of a role for AHR in alternative splicing. Our model detailing a constitutive function for AHR in WT1 regulation is supported by the finding that prevention of AHR loss pharmacologically, or by the use of the genetically-resistant D2N strain, prevented renal developmental deficits in BaP-treated metanephros, and restored normal ratios of WT1 mRNA isoforms. The complexity of AHR functions in different transcriptional complexes such as the classical AHR-ARNT, NF- 
kappa B, AP-1, and glucocorticoid receptor provides possible mechanisms for regulation of WT1 splicing (Figure 4). As mentioned in Chapter 3 , the glucocorticoid receptor associates with PGC-1, a known regulator of cotranscriptional splicing (Knutti et al., 2001). An ongoing focus of the laboratory is the possibility of direct binding of AHR to the WT1 promoter. The WT1 promoter contains two consensus AHR responsive elements within a regulatory region known to regulate transcription-coupled splicing events (Cohen et al., 1997). Wild type mK4 cells and clones expressing stable constructs for siAHR are being analyzed by chromatin immunoprecipitation for AHR-WT1 promoter associations. AHR may function to alter the splicing machinery directly (Bentley, 2002), or indirectly by altering elongation efficiency (Eperon et al., 1988; Roberts et al., 1998). Ohtake et al., presented a novel role for AHR as an E3 ligase in the proteasomal degradation of estrogen (ER) and androgen receptors $(A R)$ through associations with proteasomal complexes consisting of DDB1, ARNT, TBL3 and CUL4B, and has E3 ligase activity. We postulate that AHR may indirectly participate in proteasomal degradation of splicing factors. In fact, ER has been shown to associate with spliceosome complexes in the nucleus (Kato et al., 2005). This creates a scenario in which AHR through E3 ligase control of ER activity inherently regulates assembly and/or processivity of splicing complexes necessary for regulation of genes such as WT1 (Figure 5).

Little is known as to the exact molecular consequences resulting from alterations of the four abundant and biologically relevant isoforms of WT1. It is 
Figure 4. Schematic detailing the complexity of AHR transcriptional interactions. Depending upon cell type and stimulus AHIR can interact with different transcriptional complexes such as the classical AHR-Arnt, NF-kappa B, AP-1, and glucocorticoid receptor. 
Figure 4

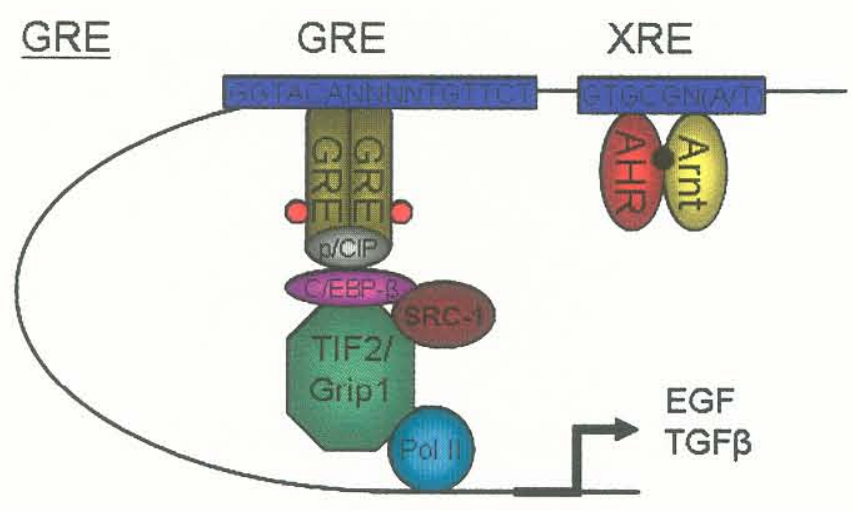


Figure 5. AHR may act as an E3 ligase in the proteasomal degradation of estrogen and androgen receptor. AHR associates with proteasomal complexes consisting of DDB1, Arnt, TBL3 and CUL4B. 
Figure 5

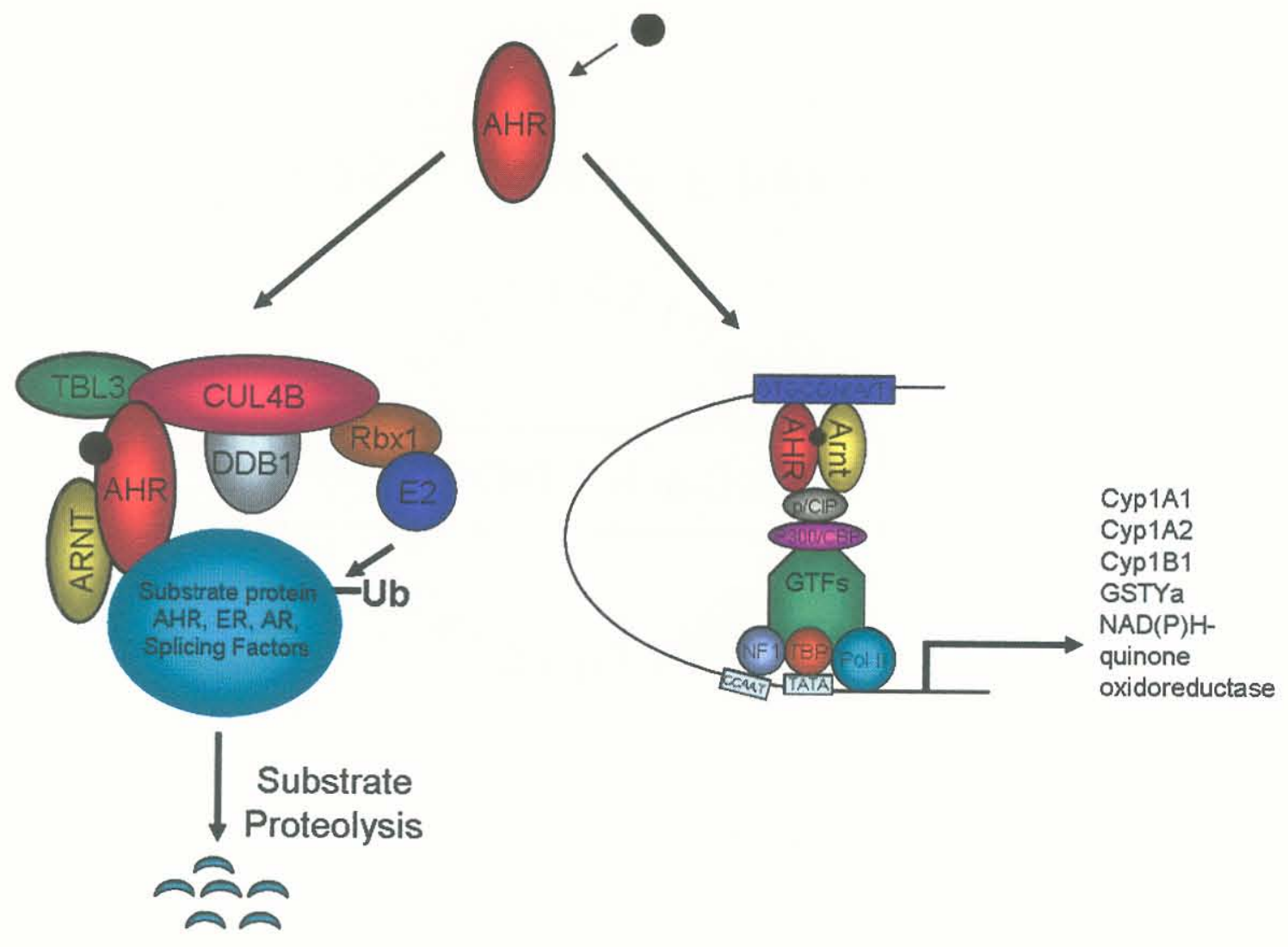


well-established that the -KTS mRNA encodes a protein that participates in transcriptional activity, and that protein encoding +KTS mRNA associates with splicing factors (Davies et al., 1998; Hastie, 2001). Mice overexpressing the KTS isoform have severely compromised renal development, evidenced by increased stromal tissue, decreases in tubular epithelium and glomerular tuft, and altered podocyte structure (Hammes et al., 2001). In both metanephros and mK4 cell cultures, BaP-induced shifts in -KTS resulted in downregulation of differentiation markers, a finding consistent with the morphological deficits seen in -KTS transgenic mice. However, a potential role of AHR in the regulation of WT1 splicing is of importance as human clinical conditions such as Denys--Drash syndrome, WAGR, and Frasier syndrome are associated with deregulation of normal splice variant ratios (Niaudet and Gubler, 2006). Restoring WT1 mRNA splice variant ratios via $A \mathrm{HR}^{\mathrm{d}}$ expression did not alter renal differentiation markers. Transgenic mice expressing solely +17 aa WT1 do not exhibit gross renal nephropathy (Natoli et al., 2002), but have deficits in other organ systems such as the olfactory system (Wagner et al., 2005).

The coordination of cell proliferation, apoptosis, adhesion, migration, differentiation, and vasculogenesis is necessary for successful ontogenesis (Larsen et al., 2001). Our data suggests a plausible role for AHR regulation of extracellular matrices $(\mathrm{ECM})$ involving cellular interactions between the ureteric bud and the metanephric mesenchyme in branching morphogenesis. ECM protein expression is tightly regulated via growth factor signaling pathways which 
in turn are regulated by master switch transcription factors. Cells stemming from the ureteric bud must digest ECM proteins to facilitate invasion. Both matrix metalloproteases (MMPs) and serine proteases are known to facilitate degradation of ECM proteins necessary in renal development. Membrane-type matrix 1 (MT-1-MMP), MMP-2, and MMP-9 have been characterized as regulators of ECM interaction in the developing kidney (Kanwar et al., 1999; Ota et al., 1998). Rat models examining the effects of maternal diabetes implicate TGF-beta as a possible MMP (Duong Van Huyen et al., 2007). In fact, cDNA array studies have revealed that many genes involved in ECM regulation and maintenance are targets for the AHR (Andreasen et al., 2006; Hillegass et al., 2006). In metanephric culture, inhibition of Smad4, a key mediator of TGF-beta, in the nephrogenic mesenchyme impairs mesenchymal induction (Oxburgh et al., 2004). Using mouse fibroblasts of varying AHR genotype, loss of AHR expression correlated to increased TGF-beta production and excretion suggesting a possible ligand-independent role in TGF-beta regulation (Chang et al., 2007). Taken together, these results implicate AHR signaling in the regulation of ECM regulatory proteins and pose an alternative mechanism for its role in renal development.

Our model of Matrigel-induced metanephric body formation agrees with the known role of (ECM) from both the ureteric bud and the metanephric mesenchyme in ureteric branching morphogenesis. Matrigel is composed of a mixture of mouse tumor derived extracellular matrix proteins including laminin, 
collagen IV, heparin sulfate proteoglycan and enactin (manufacturer's product insert). While the exact makeup of Matrigel remains unknown for proprietary reasons, studies have characterized laminin as the most abundant and reactive ingredient (Schmeichel and Bissell, 2003). This is of direct interest to our work in that laminins are key structural proteins in the renal basement membranes (Engel, 1993), and known to participate in branching morphogenesis and tubulogenesis. Laminin 1 , consisting of $\alpha-, \beta$-, and $\gamma_{\text {-subunits, is a critical }}$ component of epithelial cell polarization chiefly expressed in comma and Sshaped bodies (Durbeej et al., 1996; Ekblom, 1993). Of importance to future studies, is that exposure to anti-laminin 5 blocking antibody inhibits renal development of cultured metanephroi by preventing association with $\alpha_{3} \beta_{1}$ and $\alpha_{6} \beta_{4}$-integrin receptors (Zent et al., 2001). Recent studies have implicated integrin signaling as effectors for ECM-mediated signaling (Sonnenberg et al., 1990). In fact exposure to antibodies generated against $\alpha_{1}$, or $\alpha_{1} \beta_{1} \gamma_{1}$-subunits inhibits development of cultured metanephroi either through binding of the dystroglycan membrane complex or the $\alpha_{6} \beta_{1}$-integrin receptor (Sonnenberg et al., 1990). Ongoing work is focusing on the identification of the exact components necessary for stimulation of metanephric bodies from embryonic renal cell lines and the extent of integrin signaling.

Typically, markers of renal nephropathy such as urinary albumin/microprotein, blood urea nitrogen, and creatinine measures generally manifest in already severely compromised clinical conditions (Halperin and 
Kamel, 2000). Similarly, studies examining the embryotoxicity and teratogenicity of PAH compounds focused on overt developmental differences (Lummus and Henningsen, 1995; MacKenzie and Angevine, 1981a; Peters et al., 1999a; Rodriquez et al., 1999; Wells et al., 1997; Winn and Wells, 1997). Our findings of modest changes in glomerular number, urinary albumin, and podocyte number in response to $\mathrm{PAH}$ exposure highlight the need to reevaluate endpoints for developmental deficits and measure subtle effects which are a better estimate of human environmental exposure and risk of renal disease. Novel renal biomarkers such as RPA1, GSTYb1, and GSTMu are designed to detect nephropathy with more sensitivity than conventional methods (Falkenberg et al., 1996; Hildebrand et al., 1999; Kilty et al., 1998). Our laboratory has developed expertise in the characterization of modest, significant changes in glomerular cell populations that accompanied with traditional pathology can provide a more effective, quantitative evaluation of glomerular injury (Nanez et al., 2005; Nanez and Ramos, 2007).

The bioavailability of PAHs from exposures due to incomplete burning of organic substances such as gas, oil, wood, tobacco, and charbroiled meat present a ready source for potential regulators of development. Average PAH exposure can range from 3 to $15 \mu \mathrm{g} /$ day depending on dietary intake and are doubled with exposure to active or passive tobacco smoke (Menzie et al., 1992a; Menzie et al., 1992b; Rebagliato et al., 1995; Santodonato et al., 1981). Classical studies establishing PAHs as known embryotoxic and teratogenic 
agents used dosages of BaP ranging from $10 \mathrm{mg} / \mathrm{kg}$ to $250 \mathrm{mg} / \mathrm{kg}$, thus far exceeding even that of the highest possible human exposure (Lummus and Henningsen, 1995; MacKenzie and Angevine, 1981a; Rodriquez et al., 1999; Wells et al., 1997; Winn and Wells, 1997).

To ascertain whether our in vitro model of AHR dependent BaP-induced deficits in nephrogenesis correlated to effects obtained from environmentally relevant $\mathrm{P} A \mathrm{AH}$ exposure we chose concentrations considerably lower than those established for in utero toxicity and teratogenicity (Lummus and Henningsen, 1995; MacKenzie and Angevine, 1981a; Rodriquez et al., 1999; Wells et al., 1997; Winn and Wells, 1997). When normalized for species-dependent temporal differences in development, this exposure is representative of human exposures of at risk populations (Menzie et al., 1992a: Menzie et al., 1992b; Rebagliato et al., 1995). While the classical studies were of great mechanistic importance, recent efforts have highlighted the importance of using exposure regimens better approximating human models of environmental disease (for reviews see (BeruBe et al., 2007; $\mathrm{Ng}$ et al., 2006a; Pope, 2007)). Our findings detailing renal deficits resulting from in utero BaP exposure are unique in that they chronicle nephropathy from early post-birth through adulthood. The use of the D2N mouse model allowed us to determine that ligand binding in utero mediates $\mathrm{PAH}$ induced clevelopmental renal deficits. Our in utero studies not only implicate PAH exposure in fetal programming of adult renal diseases and ensuing complications, but provide evidence for a direct mechanism. Ongoing work will 
use genetic mouse models with mutations affecting AHR and WT1 biology to further deilineate the mechanisms of $\mathrm{PAH}$-induced renal deficits.

Our model, in which maternal $\mathrm{BaP}$ irisult results in decreases in podocyte number that impair glomerular filtration, is strikingly similar to clinical manifestations seen in conditions such as focal segmental glomerulosclerosis (FSGS) or diabetic nephropathy (Hara et al., 2001; Hayden et al., 2005; Kim et al., 2001; Wolf et al., 2005). Podocytes are terminally differentiated cells that line the glomerular basement membrane (GBM) acting as a filter mediated sizeselectivity and must structurally encompass the entire glomerular surface with foot processes to maintain proper filtration (Wiggins, 2007). Podocytopenia caused by a number of mechanism including toxic, genetic, immune, infectious and hemodynamic results in denudation of the GBM that compromise glomerular function initiating a cycle of injury and continued podocyte loss leading to progressive renal failure (Wiggins, 2007). Glomerulopathies are the most common causes of end-stage renal disease worldwide (Hricik et al., 1998). Of interest, are findings from in vivo studies in rats where weekly $10 \mathrm{mg} / \mathrm{kg} \mathrm{BaP}$ exposure of female Sprague-Dawley rats resulted in progressive elevations in total urinary protein, protein/creatinine ratios and microalbuminuria (Nanez et al., 2005). The nephropathic response involved early reductions in mesangial cell numbers coupled to podocyte injury as evidenced by effacement of foot processes. Of note was that podocytes, the only renal cell type expressing WT1 and high AHR protein amounts, in comparison to their glomerular cell types, were 
affected by BaP exposure. This suggests a scenario, currently being tested, in which PAH-induced fetal programming of renal disease may be exacerbated by repeated PAH exposures throughout adulthood. Support of this view derives from studies showing that $\mathrm{BaP}$ exacerbates nephropathy in diabetic rats (Valentovic et al., 2006). The impact of in utero BaP exposure on other glomerular cell populations such as mesangial and endothelial cells remains to be determined. However, we have obtained evidence that $\mathrm{BaP}$ compromises mesangial and podocyte cell differentiation in adult rats (Nanez et al., 2005).

Of relevance to our work is the extensive evidence linking maternal PAH exposure to fetal deficits. Some of the strongest evidence of fetal programming involves the correlation between low birth weight (LBW) and compromised renal development and initiation of the cycle involving HTN, glomerulosclerosis, nephron loss, and progressive renal failure (Celsi et al., 1998; Nwagwu et al., 2000; Sanders et al., 2005; Zandi-Nejad et al., 2006). Fetal tobacco syndrome characterized by maternal exposure to PAHs in the form of tobacco smoke results in reductions in birth weight, height, thoracic circumference (for review see (Nieburg et al., 1985). The hypothesis that active smoking morphologically alters the placenta compromising fetal capillary function resulting in a reduction of oxygen flow to the developing fetus is supported by data in both human and rodent models (Bush et al., 2000a; Bush et al., 2000b). Sasaki et al. has provided the first in vivo human evidence that AHR, CYP1A1, and GST1 genetic polymorphisms influence fetal health upon maternal tobacco smoke exposure. 
Expression of AHR protein with arg/arg at codon 211 significantly lowered birth weight and length. Interestingly, variants of enzymes regulated by AHR activity (CYP1A1 m1/m2 + m2/m2) or involved in PAH elimination (GSTM1 -/-) sensitized fetuses to the effects of maternal smoking (Sasaki et al., 2006). This study directly links PAH exposure to deficits in AHR signaling in the context of fetal programming.

The increasing bioavailability of exogenous disruptors of AHR signaling and the identification of novel endogenous AHR regulators highlights the need for a broader understanding of mechanisms by which AHR participates in renal development and cellular differentiation. Our results identify AHR-dependent glomerular deficiencies as the causative factor of in utero BaP-induced deficiencies in renal development. At the mechanistic level, these deficits are elicited via disruption of constitutive AHR signaling results in downregulation of renal cell differentiation markers, dysregulation of WT1 mRNA splice variants, and decreases in direct WT1 transcriptional targets (Figure 6).

In summary, the work presented in this dissertation defines a novel role for the AHR in renal development and in fetal programming of $\mathrm{PAH}$-induced environmental disease. Ongoing studies will require further definition of molecular interactions between AHR and WT1 and the novel specific roles of AHR in the regulation of nephrogenesis. The nature and extent of AHRregulated interactions in mediating renal developmental control that set the stage 
Figure 6. Schematic detailing renal cell differentiation. Renal cells of mesenchymal phenotype undergo a series of temporally precise genetic events that activate or repress the expression of numerous genetic elements. WT1 is a master switch that regulates the key transition from mesenchymal cells to epithelial cells. The aryl hydrocarbon receptor (AHR) is an important nuclear transcription factor both during embryogenesis and throughout maturity in multiple organisms. Evidence is presented that AHR regulates WT1 splice variant expression altering renal cell differentiation. 
Figure 6

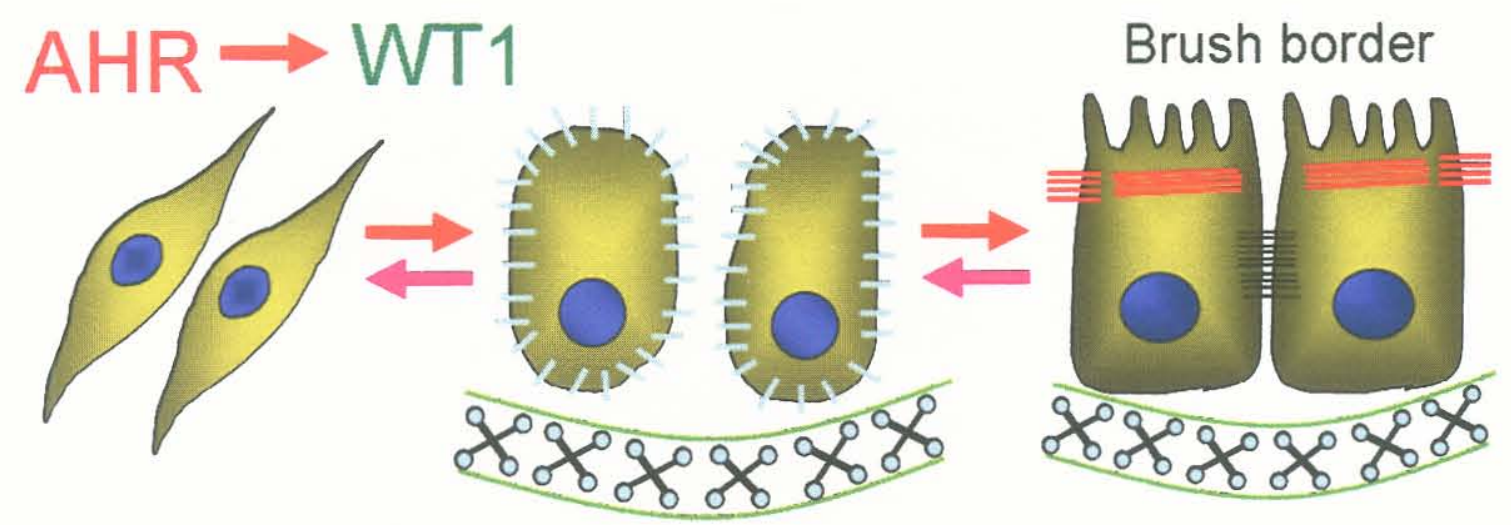

$\begin{array}{ll} & \text { E-cadherin } \\ \text { of Laminin } & \\ \equiv & \text { Tight junction }\end{array}$ 
for adult renal disease following exposure to environmental ligands of the receptor is an important hypothesis that warrants further investigation. 


\section{REFERENCES}

Abbott BD, Perdew GH, Buckalew AR, Birnbaum LS (1994). Interactive Regulation of Ah and Glucocorticoid Receptors in the Synergistic Induction of Cleft Palate by 2,3,7,8-Tetrachlorodibenzo-P-Dioxin and Hydrocortisone. Toxicol and Appl Pharmacol 128: 138.

Abrahamson DR (1987). Structure and Development of the Glomerular Capillary Wall and Basement Membrane. Am J Physiol 253: F783-94.

Alejandro NF, Parrish AR, Bowes RC, Burghardt RC, Ramos KS (2000).

Phenotypic Profiles of Cultured Glomerular Cells Following Repeated Cycles of Hydrocarbon Injury. Kidney Int 57: 1571-1580.

Andreasen EA, Mathew LK, Tanguay RL (2006). Regenerative Growth Is Impacted by TCDD: Gene Expression Analysis Reveals Extracellular Matrix Modulation. Toxicol Sci 92: 254-69.

Avner ED (1993). Renal Developmental Diseases. Semin Nephrol 13: 427-435.

Barbaux S, Niaudet P, Gubler M-C, Grunfeld J-P, Jaubert F, Kuttenn F et al (1997). Donor Splice-Site Mutations in Wt1 Are Responsible for Frasier Syndrome. Nat Genet 17: 467-470.

Barhoumi R, Mouneimne Y, Ramos KS, Safe SH, Phillips TD, Centonze VE et al (2000). Analysis of Benzo[a]Pyrene Partitioning and Cellular Homeostasis in a Rat Liver Cell Line. Toxicol Sci 53: 264-70.

Barker DJP (2004). The Developmental Origins of Adult Disease. J Am Coll Nutr 23: 588 S-595.

Barnes JD, Crosby JL, Jones CM, Wright CVE, Hogan BLM (1994). Embryonic Expression of Lim-1, the Mouse Homolog of Xenopus Xlim-1, Suggests a Role in Lateral Mesoderm Differentiation and Neurogenesis. Dev Biol 161: 168.

Barouki R, Coumoul X, Fernandez-Salguero PM (2007). The Aryl Hydrocarbon Receptor, More Than a Xenobiotic-Interacting Protein. FEBS Letters 581: 36083615. 
Bennington J, Beckwith J (1975). Tumors of the Kidney, Renal Pelvis, and Ureter. Atlas of Tumor Pathology. Armed Forces Institute of Pathology: Washington, DC.

Bentley D (2002). The mRNA Assembly Line: Transcription and Processing Machines in the Same Factory. Curr Opin Cell Biol 14: 336-42.

Bertazzi PA, Pesatori AC, Zocchetti C, Latocca R (1989). Mortality Study of Cancer Risk among Oil Refinery Workers. Int Arch Occup Environ Health 61: 261-270.

BeruBe K, Balharry D, Sexton K, Koshy L, Jones T (2007). Combustion-Derived Nanoparticles: Mechanisms of Pulmonary Toxicity. Clin Exp Pharmacol Physiol 34: 1044-1050.

Bittinger MA, Nguyen LP, Bradfield CA (2003). Aspartate Aminotransferase Generates Proagonists of the Aryl Hydrocarbon Receptor. Mol Pharmaco/ 64: 550-556.

Bowes RC, Ramos KS (1994). Responses of Vascular Smooth Muscle Cells to Toxic Insult: Cellular and Molecular Perspectives for Environmental Toxicants.

Toxicol In Vitro 8: 1151-1160.

Brophy PD, Ostrom L, Lang KM, Dressler GR (2001). Regulation of Ureteric Bud Outgrowth by Pax2-Dependent Activation of the Glial Derived Neurotrophic Factor Gene. Development 128: 4747-4756.

Buckler AJ, Pelletier J, Haber DA, Glaser T, Housman DE (1991). Isolation, Characterization, and Expression of the Murine Wilms' Tumor Gene (Wt1) During Kidney Development. Mol Cell Biol 11: 1707-1712.

Bush PG, Mayhew TM, Abramovich DR, Aggett PJ, Burke MD, Page KR (2000a). Maternal Cigarette Smoking and Oxygen Diffusion across the Placenta. Placenta 21: 824-833.

Bush PG, Mayhew TM, Abramovich DR, Aggett PJ, Burke MD, Page KR (2000b). A Quantitative Study on the Effects of Maternal Smoking on Placental Morphology and Cadmium Concentration. Placenta 21: 247-56.

Cacalano G, Farinas I, Wang LC, Hagler K, Forgie A, Moore M et al (1998). Gfralpha1 Is an Essential Receptor Component for Gdnf in the Developing Nervous System and Kidney. Neuron 21: 53-62.

Cameron JS, Blandford G (1966). The Simple Assessment of Selectivity in Heavy Proteinuria. Lancet 2: 242-247. 
Carmeliet P, Schoonjans L, Kieckens L, Ream B, Degen J, Bronson R et al (1994). Physiological Consequences of Loss of Plasminogen Activator Gene Function in Mice. Nature 368: 419-24.

Celsi G, Kistner A, Aizman R, Eklof AC, Ceccatelli S, de Santiago A et al (1998). Prenatal Dexamethasone Causes Oligonephronia, Sodium Retention, and Higher Blood Pressure in the Offspring. Pediatric Res 44: 317-322.

Chang X, Fan Y, Karyala S, Schwemberger S, Tomlinson CR, Sartor MA et al (2007). Ligand-Independent Regulation of Transforming Growth Factor \{Beta\}1 Expression and Cell Cycle Progression by the Aryl Hydrocarbon Receptor. Mol Cell Biol 27: 6127-6139.

Chesire DR, Dunn TA, Ewing CM, Luo J, Isaacs WB (2004). Identification of Aryl Hydrocarbon Receptor as a Putative Wnt/\{Beta\}-Catenin Pathway Target Gene in Prostate Cancer Cells. Cancer Res 64: 2523-2533.

Cho EA, Patterson LT, Brookhiser WT, Mah S, Kintner C, Dressler GR (1998). Differential Expression and Function of Cadherin-6 During Renal Epithelium Development. Development 125: 803-812.

Cohen HT, Bossone SA, Zhu G, McDonald GA, Sukhatme VP (1997). Sp1 is a Critical Regulator of the Wilms' Tumor-1 Gene. J Biol Chem 272: 2901-2913.

Davies RC, Calvio C, Bratt E, Larsson SH, Lamond Al, Hastie ND (1998). Wt1 Interacts with the Splicing Factor U2AF65 in an Isoform-Dependent Manner and Can Be Incorporated into Spliceosomes. Genes Dev 12: 3217-3225.

Denison MS, Nagy SR (2003). Activation of the Aryl Hydrocarbon Receptor by Structurally Diverse Exogenous and Endogenous Chemicals. Annu Rev Pharmacol Toxicol 43: 309-334.

Dong Van Huyen JP, Amri K, Belair MF, Vilar J, Merlet-Banichou C, Bruneval P et al (2003). Spatiotemporal Distribution of Insulin-Like Growth Factor Receptors During Nephrogenesis in Fetuses from Normal and Diabetic Rats. Cell Tissue Res 314: 367-379.

Duncan DM, Burgess EA, Duncan I (1998). Control of Distal Antennal Identity and Tarsal Development in Drosophila by Spineless-Aristapedia, a Homolog of the Mammalian Dioxin Receptor. Genes Dev 12: 1290-303.

Duong Van Huyen J-P, Viltard M, Nehiri T, Freund N, Belair M-F, Martinerie C et al (2007). Expression of Matrix. Metalloproteinases Mmp-2 and Mmp-9 Is Altered During Nephrogenesis in Fetuses from Diabetic Rats. Lab Invest 87: 680-689. 
Durbeej M, Fecker L, Hjalt T, Zhang HY, Salmivirta K, Klein G et al (1996).

Expression of Laminin Alpha 1, Alpha 5 and Beta 2 Chains During

Embryogenesis of the Kidney and Vasculature. Matrix Biol 15: 397-413.

Eger El, 2nd, Koblin DD, Bowland T, lonescu P, Laster MJ, Fang Z et al (1997). Nephrotoxicity of Sevoflurane Versus Desflurane Anesthesia in Volunteers. Anesth Analg 84: 160-8.

Ekblom P (1993). Basement Membranes in Development. In: Rohrbach DH and Timpl R (eds). Molecular and Cellular Aspects of Basement Membranes. Academic Press: New York. pp 359-383.

Ekblom P, Ekblom M, Fecker L, Klein G, Zhang HY, Kadoya Y et al (1994). Role of Mesenchymal Nidogen for Epithelial Morphogenesis in Vitro. Development 120: 2003-14.

Engel J (1993). Structure and Function of Laminin. In: Rohrbach DH and Timpl R (eds). Molecular and Cellular Aspects of Basement Membranes. Academic Press: New York. pp 147-171.

Englert C, Vidal M, Maheswaran S, Ge Y, Ezzell R, Isselbacher K et al (1995). Truncated Wt1 Mutants Alter the Subnuclear Localization of the Wild-Type Protein. PNAS 92: 11960-11964.

Eperon LP, Graham IR, Griffiths AD, Eperon IC (1988). Effects of RNA Secondary Structure on Alternative Splicing of Pre-mRNA: Is Folding Limited to a Region Behind the Transcribing RNA Polymerase? Cell 54: 393-401.

Everson RB, Randerath E, Santella RM, Avitts TA, Weinstein IB, Randerath K (1988). Quantitative Associations between DNA Damage in Human Placenta and Maternal Smoking and Birth Weight. J Natl Cancer Inst 80: 567-576.

Falahatpisheh MH, Nanez A, Montoya-Durango D, Qian Y, Tiffany-Castiglioni E, Ramos KS (2007). Activation Profiles of HSPA5 During the Glomerular Mesangial Cell Stress Response to Chemical Injury. Cell Stress and Chaperones.12: 209-218.

Falahatpisheh MH, Ramos KS (2003). Ligand-Activated Ahr Signaling Leads to Disruption of Nephrogenesis and Altered Wilms' Tumor Suppressor mRNA Splicing. Oncogene 22: 2160-2171.

Falahatpisheh MH, Ramos KS (2007). Ahr Transcription Factor Regulates Wt1 at the Post-Transcriptional Level and Plays a Role in Nephrogenesis (in Revision). Oncogene. 
Falkenberg FW, Hildebrand H, Lutte L, Schwengberg S, Henke B, Greshake D et al (1996). Urinary Antigens as Markers of Papillary Toxicity: Identification and Characterization of Rat Kidney Papillary Antigens with Monoclonal Antibodies. Arch Toxicol 71: 80-92.

Feld S, Hirschberg R (1996). Growth Hormone, the Insulin-Like Growth Factor System, and the Kidney. Endocr Rev 17: 423-480.

Fernandez-Salguero P, Pineau T, Hilbert DM, McPhail T, Lee SS, Kimura S et al (1995). Immune System Impairment and Hepatic Fibrosis in Mice Lacking the Dioxin-Binding Ah Receptor. Science 268: 722-6.

Fernandez-Salguero PM, Ward JM, Sundberg JP, Gonzalez FJ (1997). Lesions of Aryl-Hydrocarbon Receptor-Deficient Mice. Vet Pathol 34: 605-14.

Fujii-Kuriyama Y, Mimura J (2005). Molecular Mechanisms of Ahr Functions in the Regulation of Cytochrome P450 Genes. Biochem Biophys Res Commun 338: 311 .

Gavras I, Gavras H (2002). Angiotensin II as a Cardiovascular Risk Factor. J Hum Hypertens 16 Suppl 2: S2-6.

Goujon FM, Nebert DW, Gielen JE (1972). Genetic Expression of Aryl Hydrocarbon Hydroxylase Induction: Interaction of Various Compounds with Different Forms of Cytochrome P-450 and the Effect on Benzo(a)Pyrene Metabolism in Vitro. Mol Pharmacol 8: 667-80.

Haber DA, Timmers HTM, Pelletier J, Sharp PA, Housman DE (1992). A Dominant Mutation in the Wilms Tumor Gene Wt1 Cooperates with the Viral Oncogene E1a in Transformation of Primary Kidney Cells. PNAS 89: 6010-6014.

Hahn ME (2002). Aryl Hydrocarbon Receptors: Diversity and Evolution. Chem Biol Interact 141: 131-60.

Hahn ME, Karchner SI, Evans BR, Franks DG, Merson RR, Lapseritis JM (2006). Unexpected Diversity of Aryl Hydrocarbon Receptors in Non-Mammalian Vertebrates: Insights from Comparative Genomics. J Exp Zoolog A Comp Exp Biol 305: 693-706.

Halperin ML, Kamel KS (2000). Use of the Composition of the Urine at the Bedside: Emphasis on Physiological Principles to Provide Insight into Diagnostics and Therapeutic Issues. In: Seldin DW and Giebisch G (eds). The Kidney. Lippincott Williams \& Wilkins: Philadelphia. pp 2297-2328. 
Hammes A, Guo J-K, Lutsch G, Leheste J-R, Landrock D, Ziegler U et al (2001). Two Splice Variants of the Wilms' Tumor 1 Gene Have Distinct Functions During Sex Determination and Nephron Formation. Cell 106: 319-32.9.

Han X, Chesney RW (2003). Regulation of Taurine Transporter Gene (Taut) by Wt1. FEBS Letters 540: 71.

Hankinson $O$ (2005). Role of Coactivators in Transcriptional Activation by the Aryl Hydrocarbon Receptor. Archives of Biochemistry and Biophysics 433: 379

Hara M, Yanagihara T, Kihara I (2001). Urinary Podocytes in Primary Focal Segmental Glomerulosclerosis. Nephron 89: 342-7.

Harper PA, Wong JMY, Lam MSM, Okey AB (2002). Polymorphisms in the Human Ah Receptor. Chemico-Biological Interactions 141: 161.

Harrison ML, Nicol CJ, Wells PG (1994). Tumor Suppressor Genes and Chemical Teratogenesis: Benzo(a)Pyrene Embryopathy and Cytochromes P450 Activities in P53-Deficient Transgenic Mice. Toxicologist 14: 246.

Hastie ND (2001). Life, Sex, and Wt1 Isoforms-- Three Amino Acids Can Make All the Difference. Cell 106: 391.

Hayden MR, Whaley-Connell A, Sowers JR (2005). Renal Redox Stress and Remodeling in Metabolic Syndrome, Type 2 Diabetes Mellitus, and Diabetic Nephropathy: Paying Homage to the Podocyte. Am J Nephrol 25: 553-69.

Henry EC, Bemis JC, Henry O, Kende AS, Gasiewicz TA (2006). A Potential Endogenous Ligand for the Aryl Hydrocarbon Receptor Has Potent Agonist Activity in Vitro and in Vivo. Arch of Biochem Biophys 450: 67-77.

Henry EC, Gasiewicz TA (2003). Agonist but Not Antagonist Ligands Induce Conformational Change in the Mouse Aryl Hydrocarbon Receptor as Detected by Partial Proteolysis. Mol Pharmacol 63: 392-400.

Hewitt SM, Saunders GF (1996). Differentially Spliced Exon 5 of the Wilms' Tumor Gene Wt1 Modifies Gene Function. Anticancer Res 16: 621-626.

Hildebrand H, Rinke M, Schluter G, Bomhard E, Falkenberg FW (1999). Urinary Antigens as Markers of Papillary Toxicity. II: Application of Monoclonal Antibodies for the Determination of Papillary Antigens in Rat Urine. Arch Toxicol 73: 233-45.

Hillegass JM, Murphy KA, Villano CM, White LA (2006). The Impact of Aryl Hydrocarbon Receptor Signaling on Matrix Metabolism: Implications for Development and Disease. Biol Chem 387: 1159-73. 
Hoffer A, Chang C-y, Puga A (1996). Dioxin Induces Transcription of Fos and Jun Genes by Ah receptor-Dependent and -Independent Pathways. Toxicol Appl Pharmacol 141: 238.

Hoffman EC, Reyes H, Chu FF, Sander F, Conley LH, Brooks BA et al (1991). Cloning of a Factor Required for Activity of the Ah (Dioxin) Receptor. Science 252: 954-8.

Hohenstein P, Hastie ND (2006). The Many Facets of the Wilms' Tumour Gene, Wt1. Hum Mol Genet 15 Spec No 2: R196-201

Holderman MT, Miller KP, Ramos KS (2000). Activation of Nuclear Protein Binding to the Antioxidant/Electrophile Response Element in Vascular Smooth Muscle Cells by Benzo(a)Pyrene. Biochem Biophys Res Commun 267: 12-6.

Hosono S, Gross I, English MA, Hajra KM, Fearon ER, Licht JD (2000). ECadherin Is a Wt1 Target Gene. J Biol Chem 275: 10943-53.

Hosono S, Luo X, Hyink DP, Schnapp LM, Wilson PD, Burrow CR et al (1999). Wt1 Expression Induces Features of Renal Epithelial Differentiation in Mesenchymal Fibroblasts. Oncogene 18: 417-427.

Hotz P (1994). Occupational Hydrocarbon Exposure and Chronic Nephropathy. Toxicology 90: 163-283.

Hoy WE, Douglas-Denton R, Hughson M, Cass A, Johnson K, Bertram JF (2003). A Stereological Study of Glomerular Number and Volume: Preliminary Findings in a Multiracial Study of Kidneys at Autopsy. Kidney Int Supp/ 83: S317.

Hricik DE, Chung-Park M, Sedor JR (1998). Glomerulonephritis. N Engl J Med 339: 888-899.

Hughson M, Farris AB, III, Douglas-Denton R, Hoy WE, Bertram JF (2003). Glomerular Number and Size in Autopsy Kidneys: The Relationship to Birth Weight. Kidney Int 63: 2113.

Iben S, Royer-Pokora B (1999). Analysis of Native Wt1 Protein from Frozen Human Kidney and Wilms' Tumors. Oncogene 18: 2533-2536.

Ikuta T, Kawajiri K (2006). Zinc Finger Transcription Factor Slug Is a Novel Target Gene of Aryl Hydrocarbon Receptor. Exp Cell Res 312: 3585-94. 
Ito K, Oji Y, Tatsumi N, Shimizu S, Kanai Y, Nakazawa T et al (2006). Antiapoptotic Function of 17aa(+)Wt1 (Wilms' Tumor Gene) Isoforms on the Intrinsic Apoptosis Pathway. Oncogene 24: 2012-2020.

Jain S, Maltepe E, Lu MM, Simon C, Bradfield CA (1998). Expression of Arnt, Arnt2, Hif1[Alpha], Hif2[Alpha] and Ah Receptor mRNAs in the Developing Mouse. Mech Devel 73: 117-123.

Johnson CD, Balagurunathan Y, Tadesse MG, Falahatpisheh MH, Brun M, Walker MK et al (2004). Unraveling Gene-Gene Interactions Regulated by Ligands of the Aryl Hydrocarbon Receptor. Environ Health Perspect 112: 403412.

Kanwar YS, Ota K, Yang Q, Wada J, Kashihara N, Tian Y et al (1999). Role of Membrane-Type Matrix Metalloproteinase 1 (Mt-1-Mmp), Mmp-2, and Its Inhibitor in Nephrogenesis. Am J Physiol 277: F934-47.

Kapitulnik J, Levin W, Conney AH, Yagi H, Jerina DM (1977). Benzo[a]Pyrene 7,8-Dihydrodiol Is More Carcinogenic Than Benzo[a]Pyrene in Newborn Mice. Nature 266: 378-80.

Karavanov AA, Karavnova I, Perantoni A, Dawid IB (1998). Expression Pattern of the Rat Lim-1 Homeobox Gene Suggests a Dual Role During Kidney Development. Int J Dev Biol 42: 61-66.

Kato S, Sato T, Watanabe T, Takemasa S, Masuhiro Y, Ohtake F et al (2005). Function of Nuclear Sex Hormone Receptors in Gene Regulation. Cancer Chemother Pharmacol 56: 4-9.

Kerzee JK, Ramos KS (2000). Activation of C-Ha-Ras by Benzo(a)Pyrene in Vascular Smooth Muscle Cells Involves Redox Stress and Aryl Hydrocarbon Receptor. Mol Pharmacol 58: 152-158.

Kilty C, Doyle S, Hassett B, Manning F (1998). Glutathione S-Transferases as Biomarkers of Organ Damage: Applications of Rodent and Canine GST Enzyme Immunoassays. Chem Biol Interact 111-112: 123-35.

Kim YH, Goyal M, Kurnit D, Wharram B, Wiggins J, Holzman L et al (2001). Podocyte Depletion and Glomerulosclerosis Have a Direct Relationship in the Pan-Treated Rat. Kidney Int 60: 957-68.

Knutti D, Kressler D, Kralli A (2001). Regulation of the Transcriptional Coactivator Pgc-1 Via Mapk-Sensitive Interaction with a Repressor. PNAS 98: 9713-9718.

Kreidberg JA, Sariola H, Loring JM, Maeda M, Pelletier J, Housman D et al (1993b). Wt-1 Is Required for Early Kidney Development. Cell 74: 679-691. 
Kuure S, Vuolteenaho R, Vainio S (2000). Kidney Morphogenesis: Cellular and Molecular Regulation. Mech Develop 92: 31-45.

Ladomery M, Sommerville J, Woolner S, Slight J, Hastie N (2003). Expression in Xenopus Oocytes Shows That Wt1 Binds Transcripts In Vivo, with a Central Role for Zinc Finger One. J Cell Sci 116: 1539-49.

Lahvis GP, Lindell SL, Thomas RS, McCuskey RS, Murphy C, Glover E et al (2000). Portosystemic Shunting and Persistent Fetal Vascular Structures in Aryl Hydrocarbon Receptor-Deficient Mice. PNAS 97: 10442-10447.

Lahvis GP, Pyzalski RW, Glover E, Pitot HC, McElwee MK, Bradfield CA (2005). The Aryl Hydrocarbon Receptor Is Required for Developmental Closure of the Ductus Venosus in the Neonatal Mouse. Mol Pharmacol 67: 714-720.

Larsen WJ, Sherman LS, Potter SS, Scott WJ (2001). Human Embryology. Churchill Livingstone.

Larsson SH, Charlieu J-P, Miyagawa K, Engelkamp D, Rassoulzadegan M, Ross A et al (1995). Subnuclear Localization of Wt1 in Splicing or Transcription Factor Domains Is Regulated by Alternative Splicing. Cel/ 81: 391-401.

Lee SB, Haber DA (2001). Wilms Tumor and the Wt1 Gene. Exp Cell Res 264: 74-99.

Lee TH, Lwu S, Kim J, Pelletier J (2002). Inhibition of Wilms Tumor 1 Transactivation by Bone Marrow Zinc Finger 2, a Novel Transcriptional Repressor. J Biol Chem 227: 44826-44837.

Lestaevel P, Clarencon D, Gharib A, Peinnequin A, Cespuglio R, Gourmelon P et al (2003). Nitric Oxide Voltammetric Measurements in the Rat Brain after Gamma Irradiation. Radiat Res 160: 631-6.

Li J, Shen H, Himmel KL, Dupuy AJ, Largaespada DA, Nakamura T et al (1999). Leukaemia Disease Genes: Large-Scale Cloning and Pathway Predictions. Nat Genet 23: 348-353.

Lifton RP, Gharavi AG, Geller DS (2001). Molecular Mechanisms of Human Hypertension. Cell 104: 545-56.

Lin CQ, Bissell MJ (1993). Multi-Faceted Regulation of Cell Differentiation by Extracellular Matrix. FASEB J 7: 737-43.

Lummus ZL, Henningsen G (1995). Modulation of T-Cell Ontogeny by Transplacental Benzo(a)Pyrene. Int Immunopharmacol 17: 339-350. 
Lund AK, Goens MB, Kanagy NL, Walker MK (2003). Cardiac Hypertrophy in Aryl Hydrocarbon Receptor Null Mice Is Correlated with Elevated Angiotensin II, Endothelin-1, and Mean Arterial Blood Pressure. Toxicol Appl Pharmacol 193: 177-87.

Lund AK, Goens MB, Nunez BA, Walker MK (2006). Characterizing the Role of Endothelin-1 in the Progression of Cardiac Hypertrophy in Aryl Hydrocarbon Receptor (Ahr) Null Mice. Toxicol Appl Pharmaco/ 212: 127-35.

Maas R, Elfering S, Glaser T, Jepeal L (1994). Deficient Outgrowth of the Ureteric Bud Underlies the Renal Agenesis Phenotype in Mice Manifesting the Limb Deformity (Ld) Mutation. Dev Dyn 199: 214-28.

MacKenzie KM, Angevine DM (1981a). Infertility in Mice Exposed in Utero to Benzo(a)Pyrene. Biol Reprod 24: 183-191.

Mah SP, Saueressig H, Goulding M, Kintner C, Dressler GR (2000). Kidney Development in Cadherin-6 Mutants: Delayed Mesenchyme-to-Epithelial Conversion and Loss of Nephrons. Dev Biol 223: 38-53.

Marchand A, Tomkiewicz C, Marchandeau JP, Boitier E, Barouki R, Garlatti M (2005). 2,3,7,8-Tetrachlorodibenzo-P-Dioxin Induces Insulin-Like Growth Factor Binding Protein-1 Gene Expression and Counteracts the Negative Effect of Insulin. Mol Pharmaco/ 67: 444-452.

Marshall T, Williams K (1987). Simplified Sodium Dodecyl SulfatePolyacrylamide Gel Electrophoresis of Unconcentrated Urine with Enhanced Resolution and Detection Sensitivity. Clin Chem 33: 1886-1887.

Mathieson PW (2004). The Cellular Basis of Albuminuria. Clinical Science 107: 533-538.

McMillan BJ, Bradfield CA (2007a). The Aryl Hydrocarbon Receptor Is Activated by Modified Low-Density Lipoprotein. PNAS 104: 1412-1417.

McMillan BJ. Bradfield CA (2007b). The Aryl Hydrocarbon Receptor Sans Xenobiotics: Endogenous Function in Genetic Model Systems. Mol Pharmacol : mol.107.037259.

Menke A, Mclnnes L, Hastie ND, Schedl A (1998a). The Wilms' Tumor Suppressor Wt1: Approaches to Gene Function. Kidney Int 53: 1512-1518.

Menke AL, van der Eb AJ, Jochemsen AG (1998b). The Wilms' Tumor 1 Gene Oncogene or Tumor Suppressor Gene? Internat RevCyt 181: 151-212. 
Menzie CA, Potocki BB, Santodonato J (1992a). Ambient Concentrations and Exposure to Carcinogenic PAHs in the Environment. Environ Sci Technol 26: $1278-1284$

Menzie CA, Potocki BB, Santodonato J (1992b). Exposure to Carcinogenic PAHs in the Environment. Environ Sci Technol 26: 1278-1284.

Miller K, Ramos K (2001a). Impact of Metabolism on the Biological Effects of Benzo(a)Pyrene and Related Hydrocarbons. Drug Metab Rev 33: 1-35.

Miller KP, Chen Y-H, Hastings VL, Bral CM, Ramos KS (2000). Profiles of Antioxidant/Electrophile Resporise Element (Are/Epre) Nuclear Protein Binding and $\mathrm{C}-\mathrm{Ha}$-Ras Transactivation in Vascular Smooth Muscle Cells Treated with Oxidative Metabolites of Benzo[a]Pyrene. Biochem Pharmacol 60: 1285.

Miller KP, Ramos KS (2001b). Impact of Cellular Metabolism on the Biological Effects of Benzo[a]Pyrene and Related Hydrocarbons. Drug Metab Rev 33: 1-35.

Mimura J, Ema M, Sogawa K, Fujii-Kuriyama Y (1999). Identification of a Novel Mechanism of Regulation of Ah (Dioxin) Receptor Function. Genes Dev. 13: 2025.

Mitchell CE (1982). Distribution and Retention of Benzo(a)Pyrene in Rats after Inhalation. Toxicology Letters 11: 35

Mouchantaf R, Azakir BA, McPherson PS, Millard SM, Wood SA, Angers A (2006). The Ubiquitin Ligase Itch Is Auto-Ubiquitylated In Vivo and In Vitro but Is Protected from Degradation by Interacting with the Deubiquitylating Enzyme Fam/Usp9x. J Biol Chem 281: 38738-38747.

Mundel P, Kriz W (1995). Structure and Function of Podocytes: An Update. Anat Embryol (Berl) 192: 385-97.

Murphy LJ, Barron DJ (1993). The Igfs and Their Binding Proteins in Murine Development. Mol Reprod Dev 35: 376-381.

Murre C, Bain G, van Dijk MA, Engel I, Furnari BA, Massari ME et al (1994).

Structure and Function of Helix-Loop-Helix Proteins. Biochim Biophys Acta 1218: 129-35.

Nambu JR, Lewis JO, Wharton KA, Jr., Crews ST (1991). The Drosophila SingleMinded Gene Encodes a Helix-Loop-Helix Protein That Acts as a Master Regulator of CNS Midline Development. Cell 67: 1157-67. 
Nanez A, Alejandro NF, Falahatpisheh MH, Kerzee JK, Roths JB, Ramos KS (2005). Disruption of Glomerular Cell-Cell and Cell-Matrix Interactions in Hydrocarbon Nephropathy. Am J Physiol Renal Physiol 289: F1291-1303.

Nanez A, Ramos KS (2007). A Mutant Allele of the Aryl Hydrocarbon Receptor Protects the Developing Kidney from Hydrocarbon-Induced Deficits in Cellular Differentiation (in Preparation). Molecular Pharmacology.

Natoli TA, McDonald A, Alberta JA, Taglienti ME, Housman DE, Kreidberg JA (2002). A Mammal-Specific Exon of Wt1 Is Not Required for Development or Fertility. Mol Cell Biol 22: 4433-4438.

Nebert DW, Dalton TP, Okey AB. Gonzalez FJ (2004). Role of Aryl Hydrocarbon Receptor-Mediated Induction of the Cyp1 Enzymes in Environmental Toxicity and Cancer. J Biol Chem 279: 23847-50.

Nebert DW, Goujon FM, Gielen JE (1972). Aryl Hydrocarbon Hydroxylase Induction by Polycyclic Hydrocarbons: Simple Autosomal Dominant Trait in the Mouse. Nat New Biol 236: 107-10.

Nelson E, Goubet-Wiemers C, Guo Y, Jodscheit K (1999). Maternal Passive Smoking During Pregnancy and Foetal Developmental Toxicity. Part 2: Histological Changes. Hum Exp Toxicol 18: 257-264.

Neubert D, Tapken S (1988a). Prenatal Induction of Benzo(a)Pyrene Hydroxylases in Mice. Arch Toxicol 62: 192-199.

Neubert D, Tapken S (1988b). Transfer of Benzo(a)Pyrene into Mouse Embryos and Fetuses. Arch Toxicol 62: 236-239.

$\mathrm{Ng}$ SP, Silverstone AE, Lai Z-W, Zelikoff JT (2006a). Effects of Prenatal Exposure to Cigarette Smoke on Offspring Tumor Susceptibility and Associated Immune Mechanisms. Toxicol. Sci. 89: 135-144.

$\mathrm{Ng} \mathrm{SP}$, Silverstone AE, Lai ZW, Zelikoff JT (2006b). Effects of Prenatal Exposure to Cigarette Smoke on Offspring Tumor Susceptibility and Associated Immune Mechanisms. Toxicol Sci 89: 135-44.

$\mathrm{Ng}$ SP, Steinetz BG, Lasano SG, Zelikoff JT (2006c). Hormonal Changes Accompanying Cigarette Smoke-Induced Preterm Births in a Mouse Model. Exp Biol Med (Maywood) 231: 1403-9.

Niaudet P, Gubler MC (2006). Wt1 and Glomerular Diseases. Pediatr Nephrol 21: $1653-60$. 
Nicol CJ, Harrison ML, Laposa RR, Gimelshtein IL, Wells PG (1995). A Teratologic Suppressor Role for P53 in Benzo[a]Pyrene-Treated Transgenic P53-Deficient Mice. Nat Genet 10: 181-7.

Nieburg P, Marks JS, McLaren NM, Remington PL (1985). The Fetal Tobacco Syndrome. Jama 253: 2998-9.

Nieto MA (2002). The Snail Superfamily of Zinc-Finger Transcription Factors. Nat Rev Mol Cell Biol 3: 155-166.

Niksic M, Slight J, Sanford JR, Caceres JF, Hastie ND (2004). The Wilms' Tumor Protein (Wt1) Shuttles between Nucleus and Cytoplasm and Is Present in Functional Polysomes. Hum. Mol. Genet. 13: 463-471.

Nishinakamura R, Matsumoto Y, Nakao K, Nakamura K, Sato A, Copeland NG et al (2001). Murine Homolog of Sall1 Is Essential for Ureteric Bud Invasion in Kidney Development. Development 128: 3105-15.

Nwagwu MO, Cook A, Langley-Evans SC (2000). Evidence of Progressive Deterioration of Renal Function in Rats Exposed to a Maternal Low-Protein Diet in Utero. British Journal of Nutrition 83: 79-85.

Ohtake F, Baba A, Takada I, Okada M, Iwasaki K, Miki H et al (2007). Dioxin Receptor Is a Ligand-Dependent E3 Ubiquitin Ligase. Nature 446: 562-566.

Okey AB, Vella LM, Harper PA (1989). Detection and Characterization of a Low Affinity Form of Cytosolic Ah Receptor in Livers of Mice Nonresponsive to Induction of Cytochrome P1- 450 by 3-Methylcholanthrene. Mol Pharmacol 35: 823-830.

Olshan AF, Breslow NE, Daling JR, Falletta JM, Grufferman S, Robison LL et al (1990). Wilms' Tumor and Paternal Occupation. Cancer Res 50: 3212-3217.

Ota K, Stetler-Stevenson WG, Yang Q, Kumar A, Wada J, Kashihara N et al (1998). Cloning of Murine Membrane-Type-1-Matrix Metalloproteinase (Mt-1Mmp) and Its Metanephric Developmental Regulation with Respect to Mmp-2 and Its Inhibitor. Kidney Int 54: 131-42.

Oxburgh L, Chu GC, Michael SK, Robertson EJ (2004). Tgf\{Beta\} Superfamily Signals Are Required for Morphogenesis of the Kidney Mesenchyme Progenitor Population. Development 131: 4593-4605

Paddison PJ, Caudy AA, Bernstein E, Hannon GJ, Conklin DS (2002). Short Hairpin Rnas (ShRNAs) Induce Sequence-Specific Silencing in Mammalian Cells. Genes Dev 16: 948-58. 
Parkinson A (2001). Biotransformation of Xenobiotics. In: Klaassen C (ed). Casarett and Doull's Toxicology: The Basic Science of Poisons, 6th edn. McGraw-Hill: New York. pp 133-224.

Peters J, Narotsky M, Elizondo G, Fernandez-Salguero P, Gonzalez F, Abbott B (1999a). Amelioration of TCDD-Induced Teratogenesis in Aryl Hydrocarbon Receptor (Ahr)-Null Mice. Toxicol. Sci. 47: 86-92.

Pichel JG, Shen L, Sheng HZ, Granholm AC, Drago J, Grinberg A et al (1996). Defects in Enteric Innervation and Kidney Development in Mice Lacking Gdnf. Nature 382: 73-6.

Pinto-Sietsma S, Mulder J, Janssen W, Hillege H, de Zeeuw D, de Jong P (2000). Smoking Is Related to Albuminuria and Abnormal Renal Function in Nondiabetic Persons. Ann Intern Med 133: 585-591.

Focar P, Fischer B, Klonisch T, Hombach-Klonisch S (2005). Molecular Interactions of the Aryl Hydrocarbon Receptor and Its Biological and Toxicological Relevance for Reproduction. Reproduction 129: 379-389.

Pohl M, Stuart RO, Sakurai H, Nigam SK (2000). Branching Morphogenesis During Kidney Development. Ann Rev of Physiol 62: 595-620.

Poland A, Glover E (1990). Characterization and Strain Distribution Pattern of the Murine Ah Receptor Specified by the Ahd and Ahb-3 Alleles. Mol Pharmacol 38: 306-312.

Poland A, Palen D, Glover E (1994). Analysis of the Four Alleles of the Murine Aryl Hydrocarbon Receptor. Mol Pharmacol 46: 915-921.

Pope CA (2007). Mortality Effects of Longer Term Exposures to Fine Particulate Air Pollution: Review of Recent Epidemiological Evidence. Inhal Toxicol 19: 33 38.

Pritchard-Jones K, Fleming S, Davidson D, Bickmore W, Porteous D, Gosden C et al (1990). The Candidate Wilms' Tumour Gene Is Involved in Genitourinary Development. Nature 346: 194-7.

Puga A, Tomlinson CR, Xia Y (2005). Ah Receptor Signals Cross-Talk with Multiple Developmental Pathways. Biochem Pharmacol 69: 199.

Qin H, Zhai Z, Powell-Coffman JA (2006). The Caenorhabditis Elegans Ahr-1 Transcription Complex Controls Expression of Soluble Guanylate Cyclase Genes in the Urx Neurons and Regulates Aggregation Behavior. Dev Biol 298: 606-15. 
Ramos KS, Parrish AR (1995). Growth-Related Signaling as a Target of Toxic Insult in Vascular Smooth Muscle Cells: Implications in Atherogenesis. Life Sci 57: 627-35.

Rebagliato M, Florey CdV, Bolumar F (1995). Exposure to Environmental Tobacco Smoke in Nonsmoking Pregnant Women in Relation to Birth Weight. Am J Epidemiol 142: 531-537.

Reddy JC, Licht JD (1996). The Wt1 Wilms' Tumor Suppressor Gene: How Much Do We Really Know? Biochm Biophys Act: Reviews in Cancer 1287: 1-28

Reddy JC, Morris JC, Wang J, English MA, Haber DA, Shi Y et al (1995). Wt1Mediated Transcriptional Activation Is Inhibited by Dominant Negative Mutant Proteins. J Biol Chem 270: 10878-10884.

Reddy P, Jacquier AC, Abovich N, Petersen G, Rosbash M (1986). The Period Clock Locus of D. Melanogaster Codes for a Proteoglycan. Cell 46: 53-61.

Roberts BJ, Whitelaw ML (1999). Degradation of the Basic Helix-Loop-Helix/PerArnt-Sim Homology Domain Dioxin Receptor Via the Ubiquitin/Proteasome Pathway. J Biol Chem 274: 36351-36356.

Roberts GC, Gooding C, Mak HY, Proudfoot NJ, Smith CW (1998). CoTranscriptional Commitment to Alternative Splice Site Selection. Nucleic Acids Res 26: 5568-72.

Roberts SG (2005). Transcriptional Regulation by Wt1 in Development. Curr Opin Genet Dev 15: 542-7.

Robertson J (1998). Chemically Induced Glomerular Injury: A Review of Basic Mechanisms and Specific Xenobiotics. Toxicol Pathol 26: 64-72.

Rodriquez JW, Kirlin WG, Wirsiy YG, Matheravidathu S, Hodge TW, Urso P (1999). Maternal Exposure to Benzo(a)Pyrene Alters Development of T Lymphocytes in Offspring. Immunopharmacol Immunotox 21: 379-396.

Roquer JM, Figueras F, Botet F, Jimenez R (1995). Influence on Fetal Growth of Exposure to Tobacco Smoke During Pregnancy. Acta Paediatr 84: 118-121.

Ryan G, Steele-Perkins V, Morris J, Rauscher F, Dressler G (1995). Repression of Pax-2 by Wt1 During Normal Kidney Development. Development 121: 867 875 .

Sakurai H, Barros EJ, Tsukamoto T, Barasch J, Nigam SK (1997). An in Vitro Tubulogenesis System Using Cell Lines Derived from the Embryonic Kidney Shows Dependence on Multiple Soluble Growth Factors. PNAS 94: 6279-84. 
Sakurai H, Nigam SK (2000). Renal Differentiation and Growth. In: Seldin DW and Giebisch G (eds). The Kidney Physiology and Pathophysiology, 3rd edn. Lippincott Williams \&Wilkins: Philadelphia, PA. pp 685-702.

Sanchez MP, Silos-Santiago I, Frisen J, He B, Lira SA, Barbacid M (1996). Renal Agenesis and the Absence of Enteric Neurons in Mice Lacking Gdnf. Nature 382: 70-3.

Sanders MW, Fazzi GE, Janssen GMJ, Blanco CE, De Mey JGR (2005). High Sodium Intake Increases Blood Pressure and Alters Renal Function in Intrauterine Growth-Retarded Rats. Hyperten 46: 71-75.

Santodonato J, Howard P, Basu D (1981). Health and Ecological Assessment of Polynuclear Aromatic Hydrocarbons. J Environ Pathol Toxicol 5: 1-364.

Sariola H, Timpl R, von der Mark K, Mayne R, Fitch JM, Linsenmayer TF et al (1984). Dual Origin of Glomerular Basement Membrane. Dev Biol 101: 86-96.

Sasaki S, Kondo T, Sata F, Saijo Y, Katoh S, Nakajima S et al (2006). Maternal Smoking During Pregnancy and Genetic Polymorphisms in the Ah Receptor, Cyp1a1 and Gstm1 Affect Infant Birth Size in Japanese Subjects. Mol Hum Reprod 12: 77-83.

Saxen L (1987). Organogenesis of the Kidney.

Scharnhorst V, van der Eb AJ, Jochemsen AG (2001). Wt1 Proteins: Functions in Growth and Differentiation. Gene 273: 141-161.

Schmeichel KL, Bissell MJ (2003). Modeling Tissue-Specific Signaling and Organ Function in Three Dimensions. J Cell Sci 116: 2377-2388.

Schmidt JV, Su GH-T, Reddy JK, Simon MC, Bradfield CA (1996)

Characterization of a Murine Ahr Null Allele: Involvement of the Ah Receptor in Hepatic Growth and Development. PNAS 93: 6731-6736.

Schuchardt A, D'Agati V, Larsson-Blomberg L, Costantini F, Pachnis V (1994). Defects in the Kidney and Enteric Nervous System of Mice Lacking the Tyrosine Kinase Receptor Ret. Nature 367: 380-3.

Schuger L, O'Shea KS, Nelson BB, Varani J (1990). Organotypic Arrangement of Mouse Embryonic Lung Cells on a Basement Membrane Extract: Involvement of Laminin. Development 110: 1091-9.

Shawlot W, Behringer RR (1995a). Requirernent for Lim1 in Head-Organizer Function. Nature 374: 425-430. 
Shawlot W, Behringer RR (1995b). Requirement for Lim-1 in Head-Organizer Function. Nature 374: 425.

Shum S, Jensen NM, Nebert DW (1979). The Murine Ah Locus: In Utero Toxicity and Teratogenesis Associated with Genetic Differences in Benzo[a]Pyrene Metabolism. Teratology 20: 365-376.

Sim EU-H, Smith A, Szilagi E, Rae F, loannou P, Lindsay MH et al (2002). Wnt-4 Regulation by the Wilms' Tumor Suppressor Gene, Wt1. Oncogene 21: 29482960.

Simms LA, Algar EM, Smith PJ (1995). Splicing of Exon 5 in the Wt1 Gene is Disrupted in Wilms' Tumor. Eur J Cancer 31A: 2270-6.

Sinal CJ, Bend JR (1997). Aryl Hydrocarbon Receptor-Dependent Induction of Cyp1a1 by Bilirubin in Mouse Hepatoma Hepa 1C1C7 Cells. Mol Pharmacol 52: 590-599.

Song Z, Pollenz RS (2002). Ligand-Dependent and Independent Modulation of Aryl Hydrocarbon Receptor Localization, Degradation, and Gene Regulation. Mol Pharmacol 62: 806-16.

Song Z, Pollenz RS (2003). Functional Analysis of Murine Aryl Hydrocarbon (Ah) Receptors Defective in Nuclear Import: Impact on Ah Receptor Degradation and Gene Regulation. Mol Pharmacol 63: 597-606.

Sonnenberg A, Linders CJ, Modderman PW, Damsky CH, Aumailley M, Timpl R (1990). Integrin Recognition of Different Cell-Binding Fragments of Laminin (P1, E3, E8) and Evidence That Alpha 6 Beta 1 but Not Alpha 6 Beta 4 Functions as a Major Receptor for Fragment E8. J Cell Biol 110: 2145-55.

Stark K, Vainio S, Vassileva G, McMahon AP (1994). Epithelial Transformation of Metanephric Mesenchyme in the Developing Kidney Regulated by Wnt-4. Nature 372: 679 .

Sundberg AG, Appelkvist EL, Backman L, Dallner G (1994a). Quantitation of Glutathione Transferase-Pi in the Urine by Radioimmunoassay. Nephron 66: 162-9.

Sundberg AG, Appelkvist EL, Backman L, Dallner G (1994b). Urinary Pi-Class Glutathione Transferase as an Indicator of Tubular Damage in the Human Kidney. Nephron 67: 308-16.

Sympson CJ, Talhouk RS, Alexander CM, Chin JR, Clift SM, Bissell MJ et al (1994). Targeted Expression of Stromelysin-1 in Mammary Gland Provides 
Evidence for a Role of Proteinases in Branching Morphogenesis and the Requirement for an Intact Basement Membrane for Tissue-Specific Gene Expression. J Cell Biol 125: 681-93.

Tian Y, Rabson AB, Gallo MA (2002). Ah Receptor and Nf-[Kappa]B Interactions: Mechanisms and Physiological Implications. Chem Bio Interact 141: 97.

Torres M, Gomez-Pardo E, Dressler GR, Gruss P (1995). Pax-2 Controls Multiple Steps of Urogenital Development. Development 121: 4057-65.

Treanor JJ, Goodman L, de Sauvage F, Stone DM, Poulsen KT, Beck CD et al (1996). Characterization of a Multicomponent Receptor for Gdnf. Nature 382: 803.

Tsang TE, Shawlot W, Kinder SJ, Kobayashi A, Kwan KM, Schughart K et al (2000). Lim1 Activity Is Required for Intermediate Mesoderm Differentiation in the Mouse Embryo. Dev Biol 223: 77-90.

USDHHS (1995). Toxicological Profile for Polycyclic Aromatic Hydrocarbons. Agency for Toxic Substances and Disease Registry.

Vainio S, Lehtonen E, Jalkanen M, Bernfield M, Saxen L (1989). EpithelialMesenchymal Interactions Regulate the Stage-Specific Expression of a Cell Surface Proteoglycan, Syndecan, in the Developing Kidney. Developmental Biology 134: 382-391.

Vainio SJ, Itaranta PV, Perasaari JP, Uusitalo MS (1999). Wnts as Kidney Tubule Inducing Factors. Int J Dev Biol 43: 419-423.

Valentovic MA, Alejandro N, Betts Carpenter A, Brown Pl, Ramos K (2006). Streptozotocin (Stz) Diabetes Enhances Benzo(a)Pyrene Induced Renal Injury in Sprague Dawley Rats. Toxicol Lett 164: 214-20.

Valerius MT, Patterson LT, Witte DP, Potter SS (2002). Microarray Analysis of Novel Cell Lines Representing Two Stages of Metanephric Mesenchyme Differentiation. Mech Develop 112: 219-232.

Wada J, Liu ZZ, Alvares K, Kumar A, Wallner E, Makino H et al (1993). Cloning of cDNA for the Alpha Subunit of Mouse Insulin-Like Growth Factor I Receptor and the Role of the Receptor in Metanephric Development. PNAS 90: 10360-4.

Wagner N, Wagner K-D, Hammes A, Kirschner KM, Vidal VP, Schedl A et al (2005). A Splice Variant of the Wilms' Tumor Suppressor Wt1 Is Required for Normal Development of the Olfactory System. Development 132: 1327-1336. 
Wang ZY, Qiu QQ, Gurrieri M, Huang J, Deuel TF (1995). Wt1, the Wilm's Tumor Suppressor Gene Product, Represses Transcription through an Interactive Nuclear Protein. Oncogene 10: 1243-1247.

Wells PG, Kim PM, Laposa RR, Nicol CJ, Parman T, Winn LM (1997). Oxidative Damage in Chemical Teratogenesis. Mut Res 396: 65-78.

Wiggins RC (2007). The Spectrum of Podocytopathies: A Unifying View of Glomerular Diseases. Kidney Int 71: 1205-1214.

Winn LM, Wells PG (1997). Evidence of Embryonic Prostaglandin H SynthaseCatalyzed Bioactivation and Reactive Oxygen Species-Mediated Oxidation of Macromolecules in Phenytoin and Benzo(a)Pyrene Teratogenesis. Free Rad Biol Mede 22: 607-621.

Wolf G, Chen S, Ziyadeh FN (2005). From the Periphery of the Glomerular Capillary Wall toward the Center of Disease: Podocyte Injury Comes of Age in Diabetic Nephropathy. Diabetes 54: 1626-34.

Wong JM, Harper PA, Meyer UA, Bock KW, Morike K, Lagueux J et al (2001a). Ethnic Variability in the Allelic Distribution of Human Aryl Hydrocarbon Receptor Codon 554 and Assessment of Variant Receptor Function In Vitro.

Pharmacogenet 11: 85-94.

Wong JM, Okey AB, Harper PA (2001b). Human Aryl Hydrocarbon Receptor Polymorphisms That Result in Loss of Cyp1a1 Induction. Biochem Biophys Res Commun 288: 990-6.

Xu PX, Adams J, Peters H, Brown MC, Heaney S, Maas R (1999). Eya1Deficient Mice Lack Ears and Kidneys and Show Abnormal Apoptosis of Organ Primordia. Nat Genet 23: 113-7.

Yamazaki T, Komuro I, Kudoh S, Zou Y, Shiojima I, Hiroi Y et al (1996). Endothelin-1 Is Involved in Mechanical Stress-Induced Cardiomyocyte Hypertrophy. J Biol Chem 271: 3221-8.

Yoshino K, Rubin JS, Higinbotham KG, Uren A, Anest V, Plisov SY et al (2001a). Secreted Frizzled-Related Proteins Can Regulate Metanephric Development. Mech Develop 102: 45.

Yoshino K, Rubin JS, Higinbotham KG, Uren A, Anest V, Plisov SY et al (2001b) Secreted Frizzled-Related Proteins Can Regulate Metanephric Development. Mech Develop 102: 45-55. 
Wang ZY, Qiu QQ, Gurrieri M, Huang J, Deuel TF (1995). Wt1, the Wilm's Tumor Suppressor Gene Product, Represses Transcription through an Interactive Nuclear Protein. Oncogene 10: 1243-1247.

Wells PG, Kim PM, Laposa RR, Nicol CJ, Parman T, Winn LM (1997). Oxidative Damage in Chemical Teratogenesis. Mut Res 396: 65-78.

Wiggins RC (2007). The Spectrum of Podocytopathies: A Unifying View of Glomerular Diseases. Kidney Int 71: 1205-1214.

Winn LM, Wells PG (1997). Evidence of Embryonic Prostaglandin H SynthaseCatalyzed Bioactivation and Reactive Oxygen Species-Mediated Oxidation of Macromolecules in Phenytoin and Benzo(a)Pyrene Teratogenesis. Free Rad Biol Mede 22: 607-621.

Wolf G, Chen S, Ziyadeh FN (2005). From the Periphery of the Glomerular Capillary Wall toward the Center of Disease: Podocyte Injury Comes of Age in Diabetic Nephropathy. Diabetes 54: 1626-34.

Wong JM, Harper PA, Meyer UA, Bock KW, Morike K, Lagueux J et al (2001a). Ethnic Variability in the Allelic Distribution of Human Aryl Hydrocarbon Receptor Codon 554 and Assessment of Variant Receptor Function In Vitro.

Pharmacogenet 11: 85-94.

Wong JM, Okey AB, Harper PA (2001b). Human Aryl Hydrocarbon Receptor Polymorphisms That Result in Loss of Cyp1a1 Induction. Biochem Biophys Res Commun 288: 990-6.

Xu PX, Adams J, Peters H, Brown MC, Heaney S, Maas R (1999). Eya1Deficient Mice Lack Ears and Kidneys and Show Abnormal Apoptosis of Organ Primordia. Nat Genet 23: 113-7.

Yamazaki T, Komuro I, Kudoh S, Zou Y, Shiojima I, Hiroi Y et al (1996). Endothelin-1 Is Involved in Mechanical Stress-Induced Cardiomyocyte Hypertrophy. J Biol Chem 271: 3221-8.

Yoshino K, Rubin JS, Higinbotham KG, Uren A, Anest V, Plisov SY et al (2001a). Secreted Frizzled-Related Proteins Can Regulate Metanephric Development. Mech Develop 102: 45.

Yoshino K, Rubin JS, Higinbotham KG, Uren A, Anest V, Plisov SY et al (2001b). Secreted Frizzled-Related Proteins Can Regulate Metanephric Development. Mech Develop 102: 45-55. 


\section{CIRRUCULUM VITAE}

\section{Adrian Nañez}

\section{Contact Information}

UTMD Anderson Science Park 1808 Park Road 1C Smithville, TX 78957

Cell: (979) 574-1880

Email: adrian.nanez@gmail.com

\section{Education}

1993-1997 Alice High School

1997-2002 University of Texas at Austin

B. S. Microbiology

2002-2003 Texas A\&M University

Graduate Studies in Toxicology

2003-Pres University of Louisville

Graduate Studies in Biochemistry and Molecular Biology

\section{Experience}

1997-2002 The University of Texas at Austin

College of Pharmacy, Division of Pharmacology and Toxicology

Student Associate and Research Assistant

Mentor: John H. Richburg

Research involving male reproductive toxicology/mechanisms and regulation of testicular germ cell apoptosis.

1998 The University of Texas at Austin

College of Pharmacy, Division of Pharmacology and Toxicology National Institute of Health, Summer Minority Training Program Fellow Mentor: John H. Richburg

Program Director: Serine Lau

1999-2000 The University of Texas at Austin

College of Pharmacy, Division of Pharmacology and Toxicology

National Science Foundation, Louis Stokes Alliance for Minority

Participation in Research Fellow

Mentor: John H. Richburg

Program Director: David Laude 
UT M. D. Anderson Summer Program in Biomedical Science Fellow Mentor: Marvin Meistrich

Program Director: Michael J. Ahearn

Research studying apoptotic mechanisms involved in radiation-induced inhibition of spermatogenesis in the male reproductive system

2002-2003 Texas A\&M University

Center for Environmental and Rural Health

Graduate Studies in Toxicology

Mentor: Kenneth S. Ramos

Studies centered on the mechanism of benzo(a)pyrene induced nephropathy and inhibition of metanephric development

2003-Pres University of Louisville School of Medicine Biochemistry and Molecular Biology

Mentor: Kenneth S. Ramos

Aryl Hydrocarbon Receptor-mediated post-transcriptional control of

Wilms' Tumor Suppressor Gene and murine nephrogenesis

\section{Publications}

1. Nañez A, Ramos KS. Deregulation of fetal programming in the developing kidney by benzo(a)pyrene. (In Preparation)

2. Nañez A, Ramos KS. Loss of the aryl hydrocarbon receptor protein alters WT1 mRNA isoform expression and downstream effectors of renal cell differentiation. (In Preparation)

3. Nañez A, Ramos KS. AHR signaling and nephrogenesis: A mutant AHR allele protects the developing kidney from hydrocarbon-induced deficits in renal cell differentiation. Mechanisms of Development. (In Review). 2007

4. Falahatpisheh MH, Nañez A, Ramos KS. Post-transcriptional regulation of Wilms' Tumor Suppressor gene by Aryl Hydrocarbon receptor: A novel function during nephrogenesis for a unique bHLH-PAS homology domain transcription factor. Oncogene (In Review), 2007.

5. Falahatpisheh MH, Nañez A, Montoya-Durango D, Qian Y, Tiffany-Castiglioni $\mathrm{E}$, Ramos, KS. Activation profiles of HSPA5 during the glomerular mesangial cell stress response to chemical injury. Cell Stress and Chaperones. (In press), 2007.

6. Ramos KS, Steffen MC, Falahatpisheh MH, Nañez A. From genomics to mechanistic insight: A global perspective on molecular deficits induced by 
environmental agents. Environmental and Molecular Mutagenesis. 48(5):395-399, 2007

7. Ramos KS, Hadi Falahatpisheh, Nañez A, He Q. Modulation of biological regulatory networks during nephrogenesis. Drug Metab Rev. 38(4):677683, 2006.

8. Bozulic LD, Powell DW, Nañez A, Link AJ, Ramos KS, Dean WL. Flasma membrane $\mathrm{Ca}^{2+}$-ATPase associates with CLP36, alpha-actinin and actin in human platelets. Thromb Haemost. 97(4): 587-597, 2007.

9. Nañez A, Alejandro NF, Falahatpisheh MH, Kerzee JK, Roths JB, and Ramos KS. Disruption of glomerular cell-cell and cell-matrix interactions in hydrocarbon nephropathy. Am J Physiol Renal Physiol. 289: F1291-1303, 2005.

10. Richburg JH, and Nañez A. Fas- or FasL-deficient mice display an increased sensitivity to nitrobenzene-induced testicular germ cell apoptosis. Toxicology Letters 139: 1-10, 2003.

11. Richburg JH, Nañez A, Williams LR, Embree ME, and Boekelheide K. Sensitivity of Testicular Germ Cells to Toxicant-Induced Apoptosis in gld Mice That Express a Nonfunctional Form of Fas Ligand. Endocrinology 141: 787-793, 2000.

12. Richburg JH, Nañez A, and Gao H. Participation of the Fas-Signaling System in the Initiation of Germ Cell Apoptosis in Young Rat Testes after Exposure to Mono-(2-Ethylhexyl) Phthalate. Toxicology and Applied Pharmacology 160: 271-278, 1999.

\section{Presentations and Abstracts}

1. A Nañez ${ }^{1}, K_{\text {S Ramos }}{ }^{1}$ (2006). Aryl Hydrocarbon Receptor Participates in Posttranscriptional Control of Wilms' Tumor Suppressor Gene and Murine Nephrogenesis. Center for Genetics and Molecular Medicine, Department of Biochemistry and Molecular Biology, University of Louisville. Gordon Research Conference, Mechanisms of Toxicity.

2. A Nañez ${ }^{1}, K_{S} \operatorname{Ramos}^{1}$ (2006). Inhibition of Mouse Kidney Development by Benzo(a)pyrene: Involvement of the Aryl Hydrocarbon Receptor and Wilms' Tumor Suppressor Gene. Center for Genetics and Molecular Medicine, Department of Biochemistry and Molecular Biology, University of Louisville. Society of Toxicology, National Meeting.

3. A Nañez ${ }^{1}$, M H Falahatpisheh ${ }^{1}$, Y Qian ${ }^{2}$, E Tiffany-Castiglioni ${ }^{2}$ and KS Ramos ${ }^{1}$ (2005). Regulation of Grp78 in the glomerular mesangial cell stress response. 
Center for Genetics and Molecular Medicine, Department of Biochemistry and Molecular Biology, University of Louisville ${ }^{1}$, Department of Veterinary Anatomy \& Public Health, Texas A\&M University ${ }^{2}$. Society of Toxicology, National Meeting.

4. A Nañez, KS Ramos (2004). Modulation of renal glomerular mesangial and podocyte numbers correlates with fibronectin accumulation in benzo(a)pyrenetreated Spraque-Dawley rats. University of Louisville School of Medicine, Department of Biochemistry and Molecular Biology, Center for Genetics and Molecular Medicine. Society of Toxicology, National Meeting.

5. A Nañez, N F Alejandro, M H Falahatpisheh, M G Tadesse, and K S Ramos (2003). Cellular profiles of benzo(a)pyrene-induced nephropathy. Texas A\&M University, Center for Environmental and Rural Health. Society of Toxicology, National Meeting.

6. A Nañez, and JH Richburg (2002). Nitrobenzene-induced testicular germ cell apoptosis in young and adult FasL-deficient $(g / d)$ mice. Society of Toxicology, National Meeting.

7. CJ Giammona, A Nañez and JH Richburg (2000). Differential sensitivity of young and adult FasL mutant gld mice to mono-(2-ethylhexyl) pthalate (MEHP)-induced testicular germ cell apoptosis. Society of Toxicology, National Meeting.

8. A Nañez and J H Richburg (2000). The role of Fas signaling in testicular germ cell death. 2000 College of Natural Sciences Undergraduate Research Poster Session.

9. CJ Giammona, A Nañez and J H Richburg (1999). Differential sensitivity of young and adult Fas L mutant gld mice to mono-(2-ethylhexyl) pthalate (MEHP)-induced testicular germ cell apoptosis. Gulf Coast Society of Toxicology, Regional Meeting. Honorable Mention.

10. A Nañez and JH Richburg (2000). The role of Fas signaling in testicular germ cell death. 2000 College of Natural Sciences Undergraduate Research Poster Session.

11. A Nañez and JH Richburg (1999). The role of Fas signaling in testicular germ cell death. Louis Stokes Alliance for Minority Participation 1999 Student Research Conference.

12. A Nañez and JH Richburg (1999). Communication between two cell types in young mice testis is required for toxicant-induced germ cell death. 1999 College of Natural Sciences Undergraduate Research Poster Session. 
13. Richburg, JH, Wurm, KD, Gao, H and Nañez, A (1999). Participation of sertoli cell-expressed FasL in the initiation of germ cell apoptosis in young rat after exposure to mono-(2-ethylhexyl) phthalate (MEHP). Toxicologist 18:

\section{Honors and Awards}

2005

2005

2004-2007

2003

2001

2001-2006

2000

1999-2001

1999

1999-2002

1999

1997

1997

President University of Louisville Biochemistry Student Association Student Representative Mechanism Specialty Section, Society of Toxicology

1995 \& 1996 National Macy Scholar

1996

National Hispanic Youth Initiative Scholar 